\title{
THERMAL DATA REQUIREMENTS AND PERFORMANCE EVALUATION PROCEDURES FOR THE NATIONAL SOLAR HEATING AND COOLING DEMONSTRATION PROGRAM
}

Office of Housing and Building Technology and Thermal Engineering Section Center for Building Technology Institute for Applied Technology National Bureau of Standards Washington, D.C. 20234

\section{August 1976}

\section{Prepared for}

Energy Research and Development Administration

Division of Solar Energy

Washington, D.C. 20545

and

Department of Housing and Urban Development

Division of Energy, Building Technology and Standards

Washington, D.C. 20410

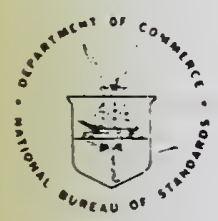

U.S. DEPARTMENT OF COMMERCE, Elliot L. Richardson, Secretary

O. Vetter, Under Secretary 

The overall goal of the National Solar Heating and Cooling Demonstration Program is "to stimulate industrial and comercial capability, including that of small businesses, to produce and distribute solar heating and cooling systems, and through widespread applications, reduce the demand on present fuel supplies." To help achieve this goal, the Demonstration Act further provides that solar heating and combined heating and cooling systems will be installed in a substantial number of buildings in the climatic regions existing in the different United States geographic areas. Evaluation of the performance and reliability of the current technology can be expedited by testing under carefully controlled conditions and by demonstration in the field.

The purpose of this document is to provide the rationale and description of the data requirements, instrumentation types and data analysis methods used to monitor and evaluate the field demonstration systems. It is recognized that complete instrumentation and subsequent technical evaluation of each system/building/climate combination cannot be performed because of the cost and data analysis limitations. Therefore, only selected unique installations will be completely instrumented and as the exact design and location of all sites becomes available, optional measurements will be made to evaluate significant features.

The report has been prepared at the National Bureau of Standards under the general guidance and review of the Solar Heating and Cooling Performance Evaluation Committee, Mr. H. J. Hale, Solar Division, Energy Research and Development Administration (ERDA), Chairman. Members of the committee include:
M. McCabe
National Bureau of Standards (NBS)
W. Christensen
Department of Defense
W. Freeborne
Department of Housing and Urban Development
A. Kromis
NASA - Marshall Space Flight Center (MSFC)
W. Littles
NASA - MSFC
F. Morse
ERDA
E. Streed (Secretary)
NBS

Specific acknowledgments are extended to Dr. Dan Ward, Colorado State University, for providing operational data and technical inputs and to David E. Galehouse, Consulting Engineer, for sensitivity and error analysis calculations. The consultation and technical reviews of Dr. J. Wayne Littles, NASA-MSFC, Dr. Mike Nash and Mr. John Bartlett, International Business Machines, and Dr. James E. Hill, NBS, have contributed significantly to this document. Thanks are also expressed to Mr. Frank Bridgers, Dr. Jack Duffie, Dr. Gerald Lowery, Prof. John Yellott, and Dr. George O. G. Löf for general review and comments regarding organization, completeness, and terminology. The encouragement and support of Mr. Jack Hale, ERDA, and Mr. Robert Dikkers, NBS/CBT, Solar Program Manager, to prepare the document in final form, is gratefully appreciated. 

This report was prepared as a reference document to prescribe a standard list of thermal performance factors and data analysis methods that can be used to evaluate solar heating and cooling systems for the National Demonstration Program. In describing these factors and the assoclated measurements, specific solar energy system designs have been identified and their features characterized to lllustrate the type and location of sensors. Inclusion of a particular design in this report in no case implies a recommendation or endorsement by the Federal government, and the presentation should not be construed as a certification that any component, subsystem, or system is preferred at the current state of technology development. Similarly, the omission of a component, subsystem, or system does not imply that the capabilities are less than those that are included. The designs presented were obtained primarily from the open literature and are intended to be used for 111 ustration purposes only.

The thermal performance data obtained from the Demonstration Program are intended to serve a variety of users including architects, engineers, manufacturers, developers and homeowners, code officials, standards writing organizations, and government planners for energy conservation, economic and bullding technology applications. An attempt has been made to identify and determine the many factors needed to satisfy the interest and needs of these diversified disciplines. However, recognizing that requirements or technology will change during the course of a five-year program, the evaluation factors and measurements can be modifled.

Comments and suggestions are welcome and should be sent to:

\author{
Manager, Solar Energy Program \\ National Bureau of Standards \\ Building 225, Room A-114 \\ Washington, D.C. 20234
}



$A_{c}$

$\mathrm{C}_{\mathrm{p}}$

COP

$F_{R}$

${ }^{h_{F G}}$

$\mathrm{K}$

$I_{T}$

$M$

$\dot{\mathrm{M}}$

$\mathrm{N}$

Q

$Q_{u}$

$\mathrm{T}$

TI or $\tau$

$\mathrm{UA}$

$\mathrm{U}_{\mathrm{L}}$

\section{GREEK LETTERS}

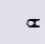

$\left(\alpha_{\tau}\right)_{e}$

$\Delta$

n

$\sigma$

$\tau_{1}$

$\tau$

\section{$\underline{\text { SUBSCRIPTS }}$}

in

out

$\Delta \mathrm{T}, \mathrm{TD}$
Collector gross area

Specific heat

Coefficient of Performance

Collector panel heat removal factor

Latent heat of vaporization

Sampling interval factor

Total incident solar radiation in plane of array

Mass

Mass flow rate

Performance index, number of samples

Cummulative thermal energy

Rate of useful energy extraction from collector

Temperature

Time

Overall heat transfer coefficient

Collector heat loss coefficient

Collector panel solar absorptance

Effective product of solar absorptance and transmittance of cover and absorber panel

Difference

Efficiency

Sensor or measurement uncertainty, standard deviation

Integration time 1 , etc.

Collector cover transmittance at air mass 2 or time
Air, ambient
Mass flow
Fluid inlet
Insolation
Inlet
Exit

Temperature difference

Sampling rate

Water, Flow 

In view of the present accepted practlce in this country for building technology, common U.S. units of measurement have been used throughout this document. In recognition of the position of the United States as a signatory to the General Conference of Weights and Measures, which gave official status to the metric SI system of units in 1960, assistance is given to the reader interested in making use of the coherent system of SI units by giving conversion factors applicable to U.S. units used in this document.

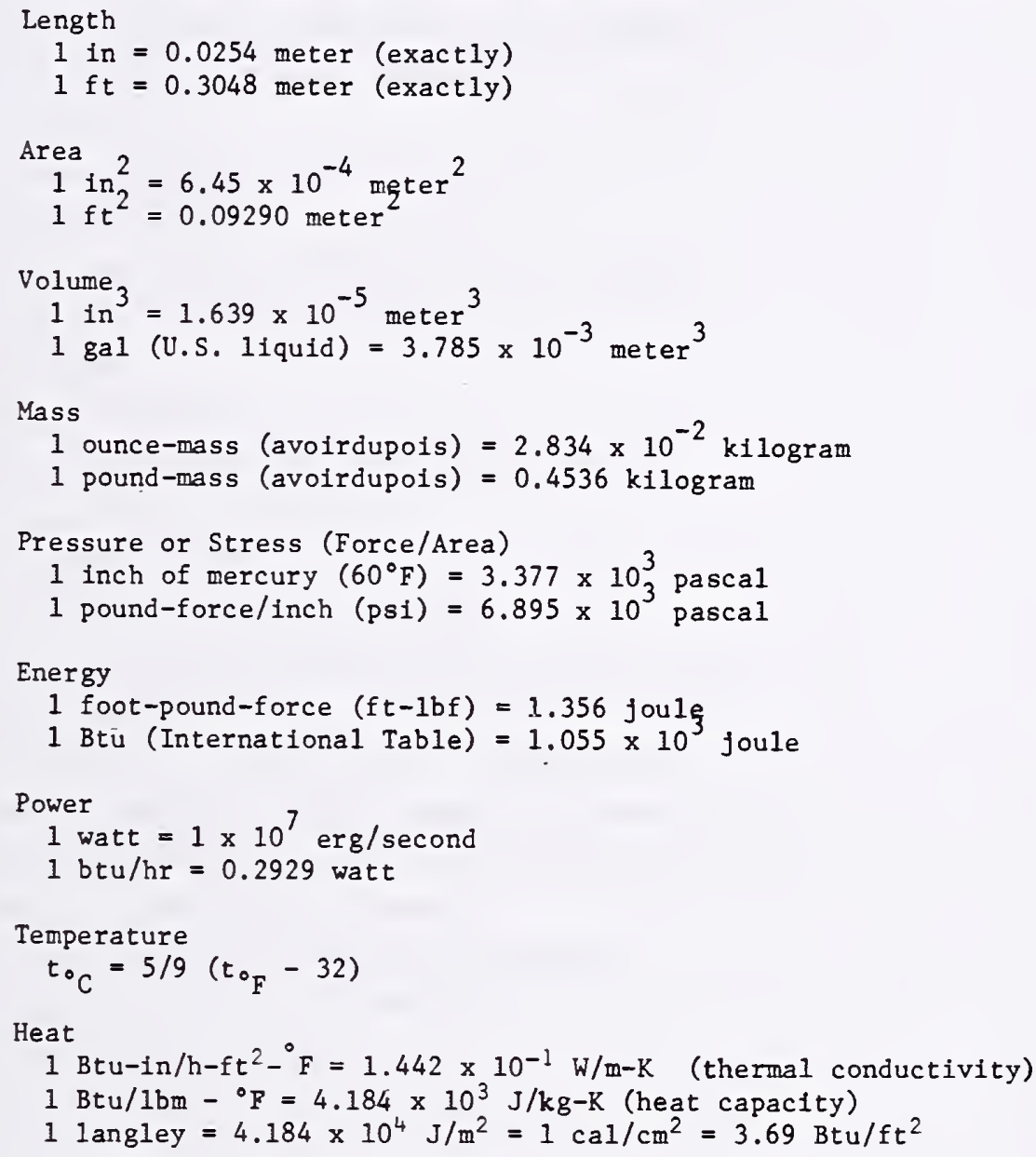



TABLE OF CONTENTS

1.0 Introduction

2.0 Program Objectives

3.0 Performance Evaluation Procedure

3.1 Standard Desionations for Sensors and Subsystems

3.2 General Solar Energy System Description and Energy Balance

4.0 Active Systems Description and Performance Calculations

4.1 Energy Collection, and Storage Subsystem

4. 2 Hot Water Subsystem

4.3 Space Heating Subsystem

4.4 Space Cooling Subsystem

5.0 Passive Systems Description and Performance Calculations 23

5.1 Roof Water Pond 25

$\begin{array}{ll}5.2 \text { Wall Collector } & 27\end{array}$

5.3 Performance Evaluation $\quad 27$

6.0 Systems Performance Evaluation Summary (Act1ve Systems) 30

6.1 Performance Factors - General 30

6.2 Energy Saved - General 32

6.3 Climatic Primary Performance Factors 33

6.4 Energy Collection and Storage Subsystem (ECCS) Primary Performance Factors

6.5 Hot Water Subsystem (HWS) Primary Performance Factors 36

6.6 Space Heating Subsystem (SHS) Primary Performance Factors 38

6.7 Space Cooling Subsystem (SCS) Primary Performance Factors 40

6.8 Solar Energy System/Building Summary 42

6.9 Secondary Performance Factors and Measurements 45

7.0 Uncertainty Analysis of Solar Performance Factors 52

7.1 Sensitivity Analysis Methods

7.2 Derivation of the Overall Instrumentation Accuracies 55

$\begin{array}{lll}7.3 \text { Summary } & 64\end{array}$

8.0 Application of Performance Factors 66

8.1 Solar Energy System Thermal Performance 66

8.2 Solar Energy System Thermal Effectiveness 66

8.3 Analytical Predictions 69

8.4 Solar Collector Array $\quad 69$

8.5 Component Predicted Performance $\quad 71$

8.6 Climatic Data 71

$\begin{array}{ll}8.7^{-} \text {Recommendations } & 74\end{array}$

9.0 Conclusions 76

10.0 References $\quad 77$ 

1. Primary Performance Evaluation Factors

2 a\&b. CSU Sampled Data Using Different Sampling Intervals

3. Range of Measured Daily Variance with Data Extrapolated for Weekly, Monthly and Yearly Periods

4. Sampling Rate as a Function of Time Interval for Several Data Requirement Types

5. Instrumentation Range, Accuracy and Measurement Frequency

6. Comparison of Collector Performance Coefficients Derived from Testing and Analysis

7. Application of Specific Performance Factors to Evaluation Areas

\section{$\underline{\text { FIGURES }}$}

1. Flow Chart of Elements Comprising Monitoring of System Performance and Operation

2. Energy Flow Diagram for a General Solar Heating, Cooling Hot Water System

3. Energy Collection and Storage Subsystem Flow Schematic, Sensors and Performance Calculations

4. Hot Water Subsystem Flow Schemat1c, Sensors and Performance Calculations

5. Space Heating Subsystem Flow Schematic, Sensors and Performance 18. 19 Calculations

6. Space Cooling Subsystem Flow Schematic, Sensors and Peformance Calculations

7. Passive Solar Energy Systems Schematics and Sensors

8. Calculation Sequence for Building Load Program 29

9. Accuracy in Calculated Solar Energy Collected (Q100) 57

10. Accuracy in Calculated Solar Used for Hot Water (Q300) 58

11. Monthly Heating and Cooling Loads and Auxiliary Energy 67 Requirement

12. Cummulative Energy Use for the MIT Solar House IV During 68 Two Winter Heating Seasons

13. Comparison of Collector Panel and System Collector Array 70 Performance

14. Illustration of Detecting Collector Degradation from Thermal Performance Measurements 

DATA REQUIREMENTS AND THERMAL PERFORMANCE EVALUATION PROCEDURLS FOR THE

NATIONAL SOLAR HEATING AND COOLING DEMONSTRATION PROGRAM

A major objective of the Solar Heating and Cooling Demonstration Program, described In ERDA-23A [1], is to provide data on the technical performance of solar heating and cooling systems.* This data, following its cullectior, analysis, and evaluation will be used to provide the information and data base needed to evaluate subsystem and system performance, to develop definitive performance criteria and to formulate analytical models lor use as design guidelines for solar heating and cooling systems. This report is intended to provide a description and rationale for the instrumentation selection and resultant data required to monitor and evaluate the thermal effectiveness and reliability of solar heating, cooling and hot water systems in meeting bullding thermal loads and in conserving conventional fuels or energy.

A basic assumption utilized in the preparation of this report concerns the purpose of the data to be acquired by the described instrumentation. Because of the demonstration nature of the program, the data acquired must be sufficient to allow for the evaluation of the thermal effectiveness of the solar components and system, but is not intended to be utilized directly in the development of specific components.

Because nationally accepted test procedures for bullding heating and cooling systems do not exist at the present time and because of the relatively large number of combinations possible for solar and conventional HVAC systems, the data requirements, measurement procedures and equations used to determine the performance factors dicussed in this report will require modifications dictated by the characteristics of the specific system belng evaluated.

The approach and methodology employed to obtain, evaluate and compare thermal performance data is shown in Figure 1. Measurements from the on-site sensors are used to determine the solar system energy contribution, the auxiliary energy requirements, the building heating or cooling load, the climatic conditions and the comfort level maintained by the system. Selected buildings and certain type solar systems will be analytically modeled to predict system performance and building thermal response as a function of the measured climatic conditions. The resulting predictions will then be compared with the experimental data and the analytical modeling procedures validated. Validated analytical models will be used to predict performance on similar solar system/building types having either reduced levels of instrumentation or none at all.

* Solar heating and cooling systems are referred to as Solar Energy Systems in this report. 



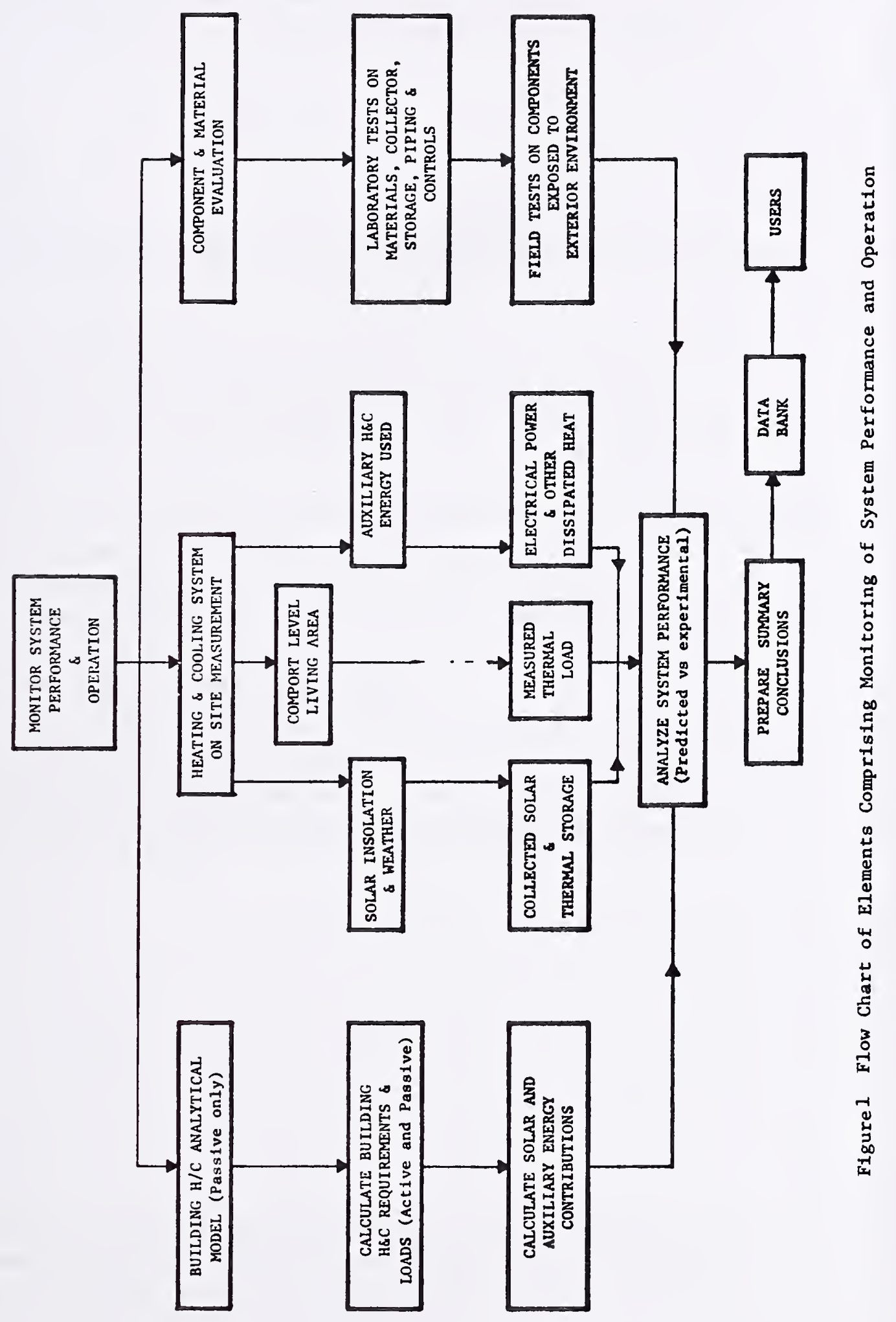



Additional laboratory or field test data on critical components as a function of time and operating conditions should also be made to the extent necessary to characterize their performance and resolve differences between predicted and measured system performance.

\subsection{PROGRAM OBJECTIVES}

Technical performance evaluation of each solar energy system/building/climatic region demonstration will be based upon the following factors:

1. Determining the savings in fossil fuel and electrical energy resulting from the use of solar energy for space heating, space cooling and/or hot water.*

2. Determining the total heating, cooling and/or HW thermal energy loads and the fraction of each load supplied by solar energy for monthly, seasonal and/or annual periods.

3. Measuring the solar energy system efficiency for converting solar radiation into useful thermal energy for monthly and seasonal or annual periods.

4. Measuring the thermal performance of major subsystems or components and the thermal interactions between collector array, storage and energy conversion equipment

5. Measuring the occupants use of the system by means of parameters such as the temperature level maintained and hot water demand.

6. Determining the major system operational characteristics and degradation over the life of the demonstration ( 1 to 5 years).

7. Obtaining records of the incident solar radiation and other pertinent site environmental nparameters that could affect the performance of the system over the life of the demonstration.

* The term hot water (HW) as used in this report includes both residential domest1c hot water (DHW) and comercial service hot water (SHW). The commercial service hot water may either be potable or nonpotable depending on its intended use. 

Performance factors and associated data requirements have been classificd into three categories. Category one (primary) items are required for a data summary that is considered essential to adequately measure the solar energy system or subsystem thermal effectiveness and determine the energy saved by the solar energy system in comparison with the energy that would have been used by a conventional hot water, space heating, or space cooling systems. Without this data, comparative evaluations of different solar energy subsystems and systems would be incomplete or impossible. These primary performance evaluation factors are shown in Table 1 and described in detail in Section 6.0.

Category two (secondary) requirements are for data deemed important and useful in evaluating different subsystems or components. Such data make it easier to understand the component interactions that occur in system operation and serve as an aid in comparative analysis or simulation but are not essential. In general second category data can be determined by appropriate calculations or approximations using category one measurements however in some cases the data can on $1 \mathrm{y}$ be direct measurements.

An example of a secondary performance factor is the storage efficiency. The average storage medium temperature is the significant parameter in determining the amount of stored energy avallable. The change of storage medium temperature during a time period with no addition or withdrawal of energy can provide a measure of the storage efficiency. The storage medium temperature is thus very useful but is not unique to the system thermal evaluation.

Category three data are obtained from special measurements which are not partioularly essential for current analysis needs, but which serve to define system operational conditions. Wind direction and velocity are examples of such measurements. The building load, particularly infiltration, and collector losses are related to wind effects. However, most analyses to data have not incorporated corrections or used detailed calculations to correlate performance with wind data.

3.1 Standard Designations for Sensors and Subsystems

In order to standardize the performance calculations and identify sensors according to type and location, an alpha-numeric name is provided for each performance factor and sensor. A five character name is used consisting of one or two letters which designate either the sensor type or the measured or calculated quantity and a three digit number which identifies the subsystem or data group as follows: 



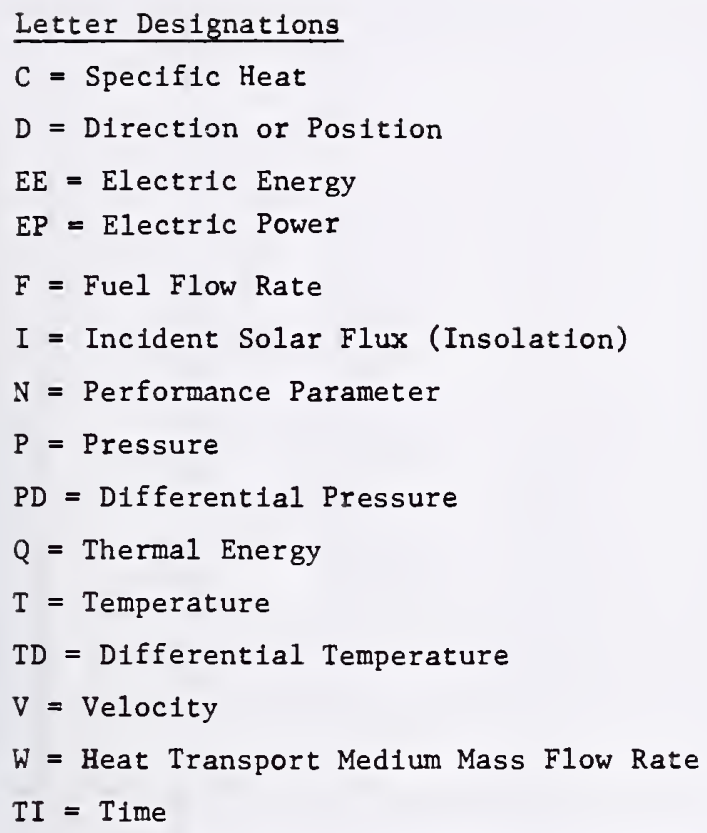

\section{Subsystem Designations}

\begin{tabular}{ll} 
Number Sequence & Subsystem/Data Group \\
\hline 001 to 099 & Climatological \\
100 to 199 & Collector and Heat Transport \\
200 to 299 & Thermal Storage \\
300 to 399 & Hot Water \\
400 to 499 & Space Heating \\
500 to 599 & Space Cooling \\
600 to 699 & Building/Load
\end{tabular}

Thus the sensor designation T101 defines an absolute temperature measurement in the collector subsystem and the variable name Q600 defines a heat flow measurement or calculation for a bullding load grouping.

General Solar System Description and Energy Balance Prior to discussing the performance evaluation and measurement requirements of solar energy systems, it is useful to describe in general terms the equipment and subsystems that comprise a solar energy system and to describe the flow of thermal energy from the solar equipment, through the energy conversion and distribution equipment to the bullding. As shown in Figure 2, the basic elements of a solar hot water, space heating and space cooling system include a solar energy collection and storage subsystem (ECSS), an energy conversion and distribution subsystem (ECDS) and the building. 



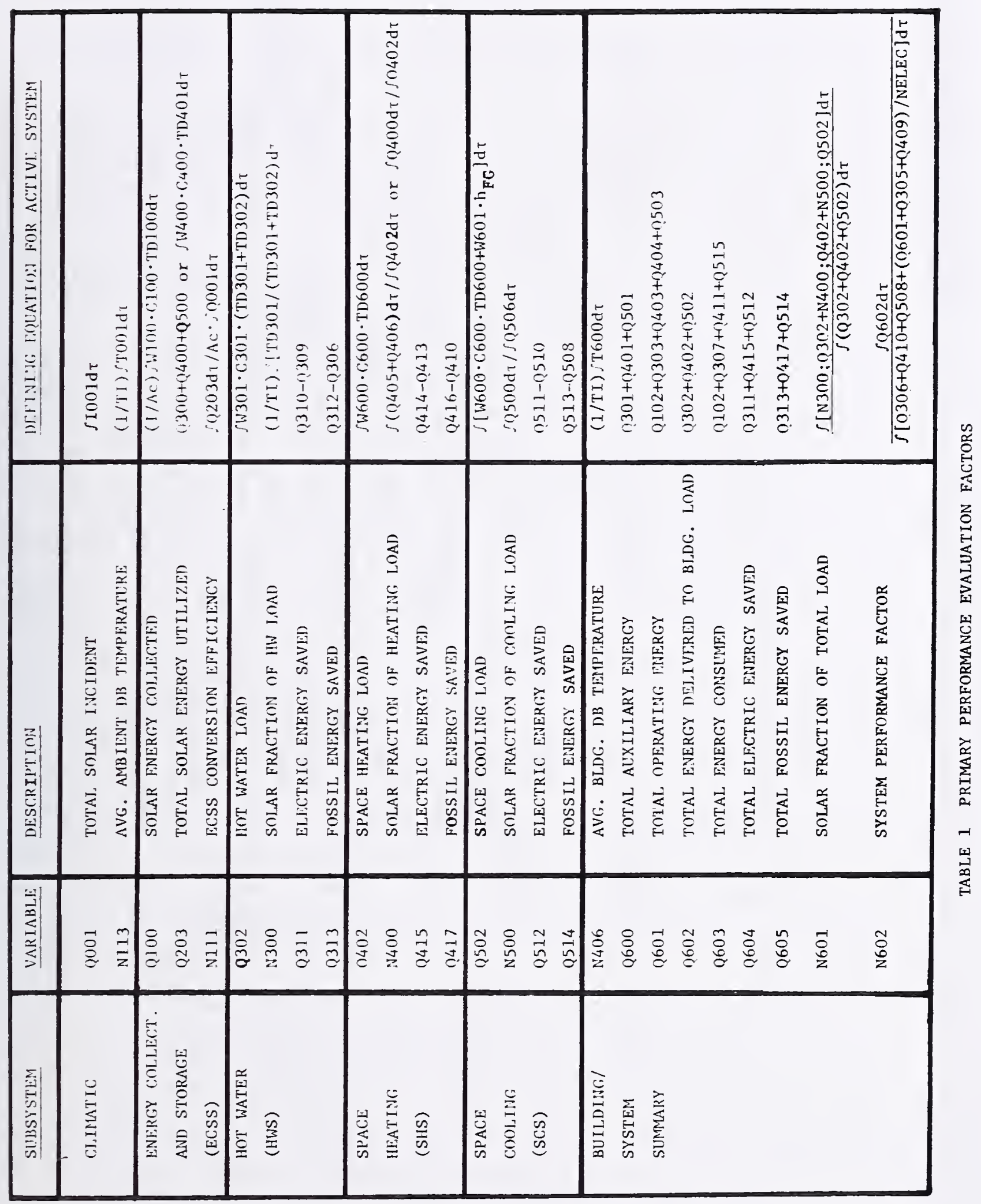





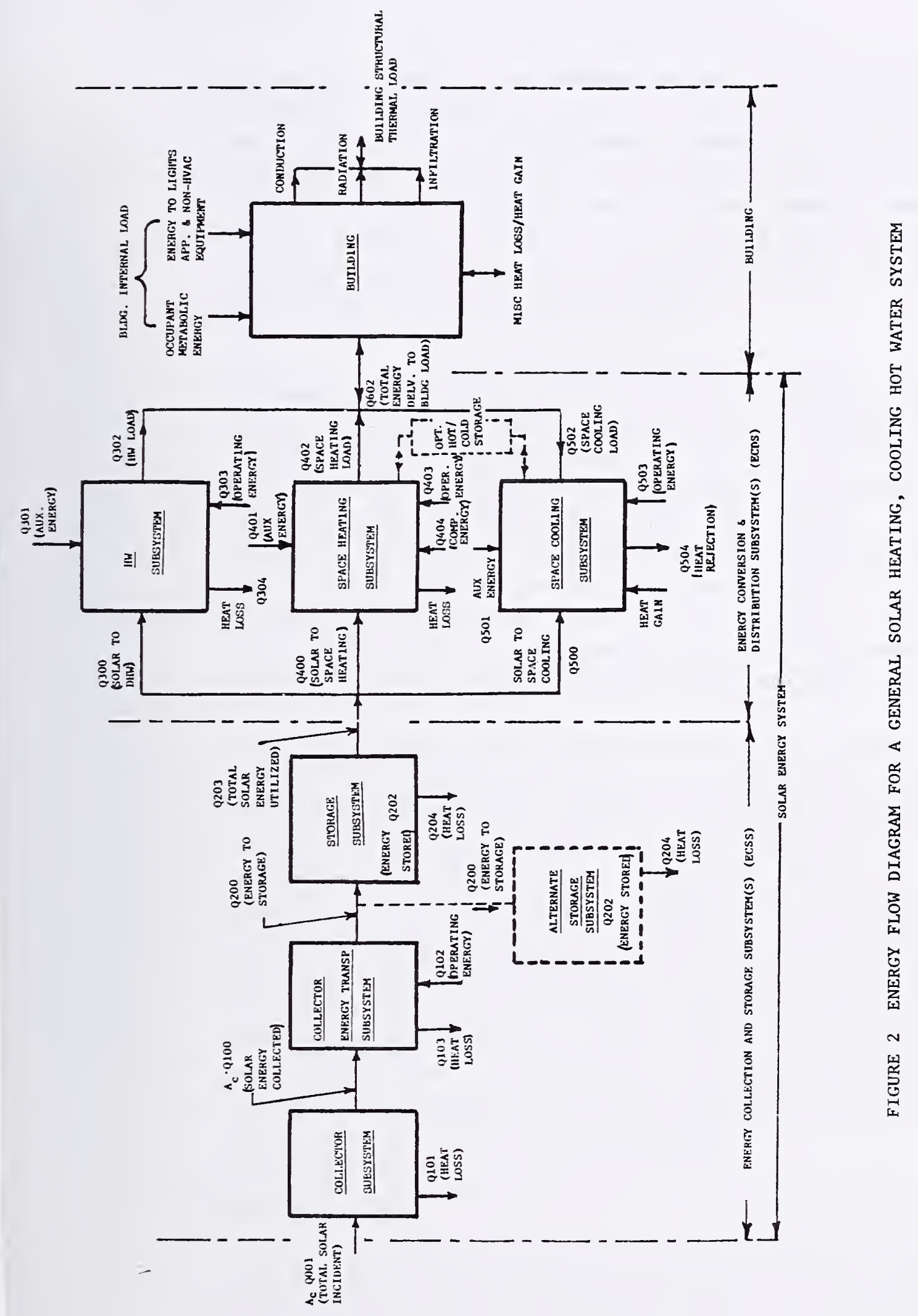



The function of the collector subsystem, collector energy transport subsystem, and storage subsystem (ECSS) is to convert the relatively variable incident solar radiation to a relatively steady source of thermal energy in the form of elevated temperature heat transport fluid or storage medium. This solar source acts as a significant thermal energy source for the bullding's energy conversion equipment. The major purpose of the ECSS is to reduce the consumption of non-renewable energy sources such as natural gas, oil, and electricity normally used to provide the hot water, heating, and cooling for the building.

The energy conversion and distribution subsystem is comprised of three subsystems to provide the distinct functions of HW heating, space heating and space cooling and ut lilizes conventional HVAC equipment such as electric or fuel fired heating furnaces, hot water heaters, heat pumps, absorption chillers and their associated pumps, fans, heat exchangers, controls, piping and ductwork. The function of this equipment is to combine the energy avallable from the solar subsystem with the auxiliary energy available from the conventional energy sources when the supply of solar energy is inadequate, and to convert the solar energy to a useful energy form for the building. To accomplish this conversion and distribution function, additional electrical energy is required to power the pumps, fans, and controls.

The building consists of the various structural elements in which of thermal energy between the outdoor and indoor environments occurs primarily by the process of conduction, convection, radiation, and infiltration. When the solar heat gain and the structural heat losses and gains are combined with the internal heat gains from the lights, appliances and other equipment and the metabolic heat from the occupants, and these loads are absorbed by the air in the temperature controlled spaces of the bullding, they comprise the building thermal load. If the HVAC equipment's rate of heat removal or addition to the building is exactly equal to the building thermal load, the air temperature is stabilized and the building is in balance. In the context of this report, hot water is also treated as a building thermal load in that the HW subsystem capability to provide the thermal energy required at the desired temperature must be balanced against the actual rate of hot water consumption.

\subsubsection{Thermal Energy Flow}

A primary tool which can be used in the location and choice of measurements is the concept of heat balances. For a given component, the amount of energy input must equal the energy output plus the change in stored energy within the component. 

This tool can be particularly useful as a check on the installed instrumentation. By obtaining the heat balance periodically on a component or subsystem, evaluation of the losses and accuracy of the installed data instrumentation can be made. On1y when the heat balance "error" is no longer within acceptable limits will selected investigation (on the particular subsystem) be required to determine the need for sensor recalibration or subsystem maintenance.

The performance evaluation factors can be defined in terms of the thermal energy quantities shown for the generalized system of Figure 2, in which an arrow leading into a box represents the net flow of a particular thermal energy quantity into a subsystem. The quantities shown represent the integrated rate of thermal energy flow over a sufficient period of time such that thermal storage in each subsystem (with the exception of the storage subsystem) is negligible. For example, in Figure 2 the quantity Q203 represents the net flow of thermal energy out of the ECSS system and into the energy conversion and distribution subsystem. The quantity Q602 represents the net flow of thermal energy between the building and the energy conversion and distribution subsystem; i.e., the total building energy load.

\subsubsection{Subsystem Heat Loss}

Examination of Figure 2 indicates a quantity called "heat loss" (or "heat gain") associated with each subsystem element. This quantity represents the difference between the total energy that originally entered the subsystem and the thermal energy delivered by the subsystem. In most cases, the subsystem heat loss represents thermal energy transferred to the subsystem environment by heat loss through the component insulation.

Depending on the physical location of the component, the subsystem environment can be outside the building either above or below ground or inside the building either in a temperature controlled or a non-controlled space. No further use is made of the heat lost by components located outside the bullding and above ground. However, the heat lost by components in the other locations can affect the rerformance of the solar energy system.

For example, with a burled non-insulated storage unit some heat lost to the environment when the storage medium temperature is relatively high may eventually be recovered when the storage medium temperature is low. In addition, for those components located within the building, some or all of the subsystem's heat loss may find its way into the temperature controlled portion of the building which is shown in Figure 2 as a miscellaneous heat loss/heat gain for the building. The entire subsystem heat loss for those components located within a temperature 

controlled space will be effective in reducing the building's heat load during the heating season and in increasing the building's cooling load during the cooling season.

In the case of a subsystem having components located in a non-temperature controlled room in a bullding, the effects of subsystem heat loss on the solar energy system performance are difficult to assess. The heat loss from a storage unit located in an unheated basement will certainly raise the alr temperature of the room, which will reduce the heat losses for the heating equipment and increase the heat gains for the cooling equipment located in that room. However, little benefit of the subsystem heat loss will be realized unless specific means are provided to utilize the heat loss to reduce the building heating requirements.

Examination of each subsystem element in Figure 2 reveals the energy quantities that must be either measured or estimated to determine the subsystem heat balance. Subsystem heat loss is probably the most difficult quantity to measure, therefore, it must be determined from the heat balance by measuring or calculating all the other quantities.

In the following description of subsystem equipment measurements and performance evaluation, the basic approach to performance measurement is to instrument all energy flow quantities except subsystem heat loss and to determine this quantity by the energy balance method. Additional instrumentation is recommended for the solar collector subsystem and storage subsystem to enable correlation of heat loss with observed temperature differentials and thus more effectively monitor these important subsystems. It is recognized that this approach to instrumentation may not always be practical, because of other constraints such as sensor cost, rellability and performance, avallable data channels, etc. When such reductions In measurement are considered, it will be necessary to assess the impact on overall evaluation and accuracy of the stated objectives of performance analysis.

In the subsequent sections describing the performance evaluation of solar energy systems, the performance equations are developed on the basis that subsystem heat loss does not affect the performance of a solar energy system with respect to such prinary evaluation factors as energy saved, heating and cooling load, and the solar fraction of the heating and cooling load. However, it is necessary that the heat loss for solar energy transport and storage subsystems located in temperature controlled spaces be determined. When significant losses are calculated, the pertinent equations (energy saved, heating and cooling load, and solar fraction of the heating and cooling load) must be modifled to indicate heat loss effects. 

The following section describes the flow schematic drawings and instrumentation requirements for several typical solar energy systems, which are used for hot water, space heating and space cooling, to illustrate the methods of evaluating performance. The objectives of this section are to define the various subsystems sufficiently to enable an analyst to convert a site contractor's solar energy system mechanical drawings (showing the equipment, piping, ducting, controls etc.) into the various system, subsystems and components as shown in Figures 2, 3, 4, 5 and 6 . The location and type of sensors can then be selected and the performance evaluation equations defined based on the guidelines established herein.

In order to fllustrate the sensor locations and performance evaluation factors, the subsystem component configurations shown in Figures 3, 4, 5 and 6 were assumed to represent reasonable subsystems that are capable of utilizing solar energy to reduce the consumption of conventional energy. It is recognized that numerous variations on these subsystem and component configurations are possible. It is therefore re-emphasized that the schematic drawings are illustrative only and should not be interpreted as recommendations by the government as to the most efficient means to use solar energy to reduce conventional energy consumption in satisfying the hot water heating and space heating and cooling requirements of buildings.

In order to provide some flexibility in the selection of sensors, three different categories of performance evaluation have been defined; primary, secondary and special. Primary factors are deemed mandatory for each system and therefore all sensors used to calculate primary performance factors must be provided. Secondary performance factors are desireable and these data will normally be provided as a fallout of the data provided with the primary sensors, however, whenever additional non-primary sensors are required to calculate a secondary performance factor, the requirement to provide the additional sensor is optional. Special performance factors are calculated from data taken with special sensors, which are provided as determined by the needs of each particular system and site.

\subsection{Energy Collection and Storage Subsystem}

Figure 3 describes the flow schematics, instrumentation and performance calculations required to characterize two alternate energy collection and storage subsystems (ECSS) and to define the required sensor locations and performance evaluation factors.

Subsystem elements common to both systems include a collector subsystem, energy transport subsystem and a storage subsystem. In System $A$, the heat transfer medium is a liquid and the storage subsystem is in series with the collector subsystem and the energy conversion and distribution subsystem (ECDS), therefore all the 


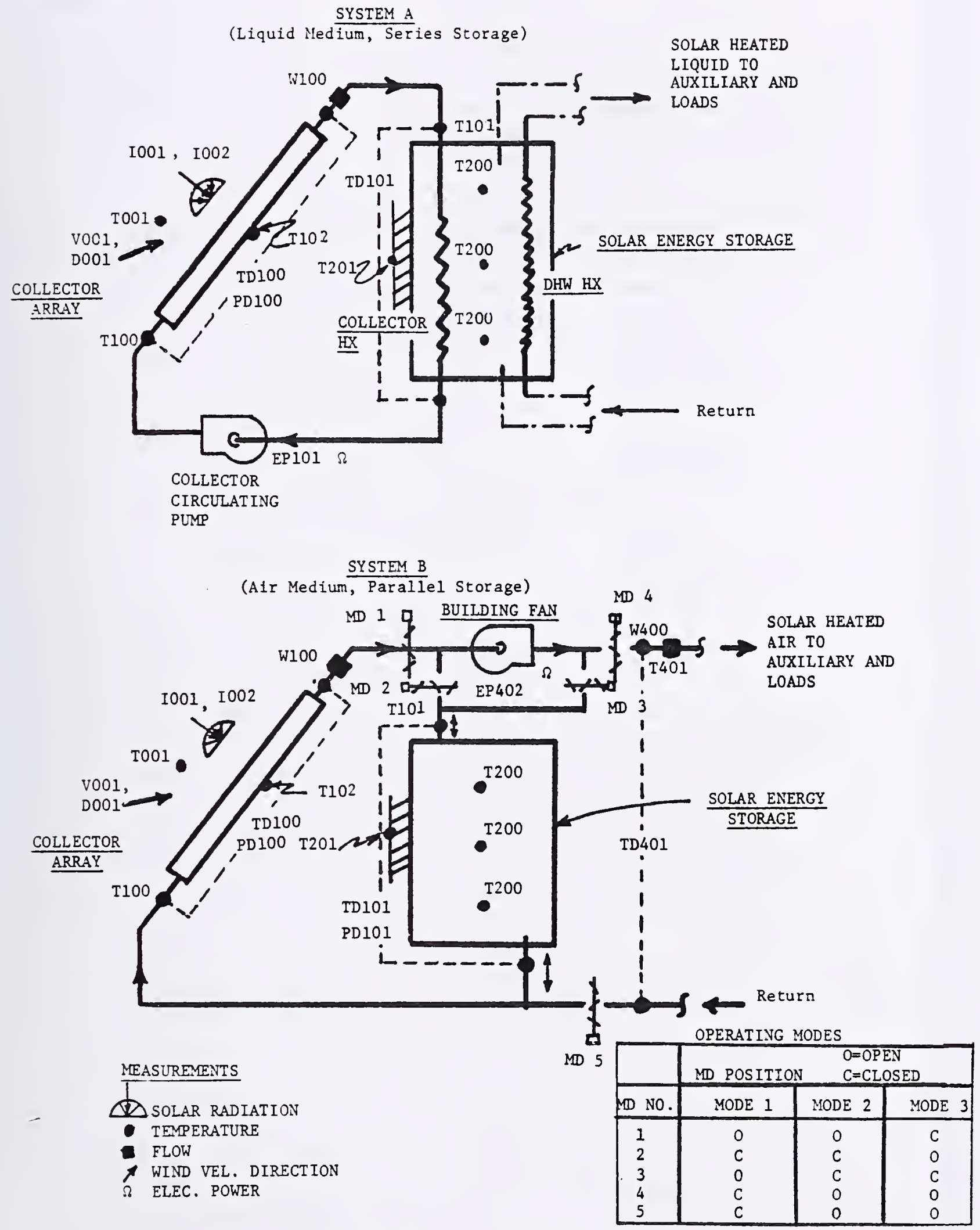

FIGURE 3 SPACE HEATING SUBSYSTEM FLOW SCHEMATICS AND SENSORS 


thermal energy collected is transferred through the storage tank before going to the ECDS. In System B, the heat transport medium is air and the storage subsystem is in parallel with the collector subsystem and the ECDS, thus permitting collected solar energy to be transferred to either the storage subsystem or to the load.

In System A, during sunny periods the collector circulation pump circulates the heat transport fluid through the collector array where it is heated by absorbed solar radiation. The absorbed energy is transferred to the storage tank via the collector heat exchanger.

In SYSTEM B a fan combined with five motorized dampers (MDS) permits operation of the system in three different modes. In Mode 1 on a sunny day when there is no demand for space heating, the fan circulates air between the collector and storage unit and thereby temporarily stores thermal energy for future use. In Mode 2 when a demand exists for space heating and there is solar radiation avallable, the fan circulates air between the collector and the building load. In Mode 3 when a demand exists for space heating and there is insufficient solar radiation available, the fan circulates air between the storage unit and the bullding load and thus makes avallable the previously stored thermal energy for space heating. In Modes 2 and 3, whenever the building heating load is not satisfied by the avallable energy either from the collector or from storage, a final stage of heating is provided by an auxiliary source using conventional fossil fuel or electrical energy.

To obiain data for full evaluation of all performance factors, the sensor types and locations shown in Figure 3 are required. These include the sensors for a local weather station which consists of total (direct plus diffuse) solar radiation at the solar array tilt angle and ambient dry bulb temperature. Wind velocity and direction sensors are provided on certain selected sites as special measurements. On solar energy systems using concentrating collectors, an additional sensor measurlng diffuse radiation is also required. The collector and heat transport subsystems have temperature sensors at the collector array inlet, on the absorber plate of one panel and at the collector heat exchanger inlet in addition to differential temperature sensors across the collector array and collector heat exchanger. The storage subsystem temperatures include the average storage medium and exterior ambient temperatures. Storage subsystems utilizing stratification to improve performance should measure interior temperatures at specific locations rather than average storage medium temperature. 

In SYSTEM A, a single mass flow sensor is required to determine the liquid flow rate between the collector and storage subsystems. In SYSTEM $B$, two mass flow sensors are required. One flow sensor measures the air flow rate through the collector and the second sensor measures the air flow rate to the load. The air flow rate to the storage system is determined by the difference in flow rate to the two sensors, assuming negligible air leakage. Operating power measurement is required for the circulating pump of SYSTEM $A$, or the building fan of SYSTEM $B$ and for the operating controls of both systems. Since the building fan of SYSTEM B also provides the energy to move air through the non-solar equipment and the building, only a portion of this power is charged against the solar energy system. Pressure differential sensors are provided across the collector arrays in both systems and across the storage unit in SYSTEM B as special measurements.

Figure 3 also defines the performance calculations necessary for each type of energy system. As indicated, many of the equations are appropriate for both system types and where different equations are required, the particular system is noted. However, not all active solar system types to be considered for the demonstration program can be described by the listed equations. In that case, the equations must be rewritten as required, to define the desired performance factors for the specific systems to be evaluated. In addition to the performance calculations noted, several of the calculated quantities are considered of special significance and are categorized as primary performance evaluation factors. These varlables will be discussed in greater detall in Section 6 .

4.2 Hot Water Subsystem

Figure 4 shows the flow schematic, instrumentation requirements and performance calculations required for the HW subsystem. The subsystem selected for 1llustration consists of the HW storage tank, circulation pump, auxiliary heat exchanger, piping and controls but does not include the tW heat exchanger which is located within the solar storage tank and is therefore considered a part of the storage subsystem. (Had the HW heat exchanger been located on the outside of the solar storage tank, it would have been considered a part of the HW subsystem).

In operation, heat is transferred from the storage subsystem to the HW storage tank by circulating potable water through the HW heat exchanger which is located in the storage tank. Whenever a demand is made for hot water, solar preheated water is withdrawn from the top of the HW storage tank and replaced by cold make-up water at the bottom. If the temperature of the water leaving the HW tank is 



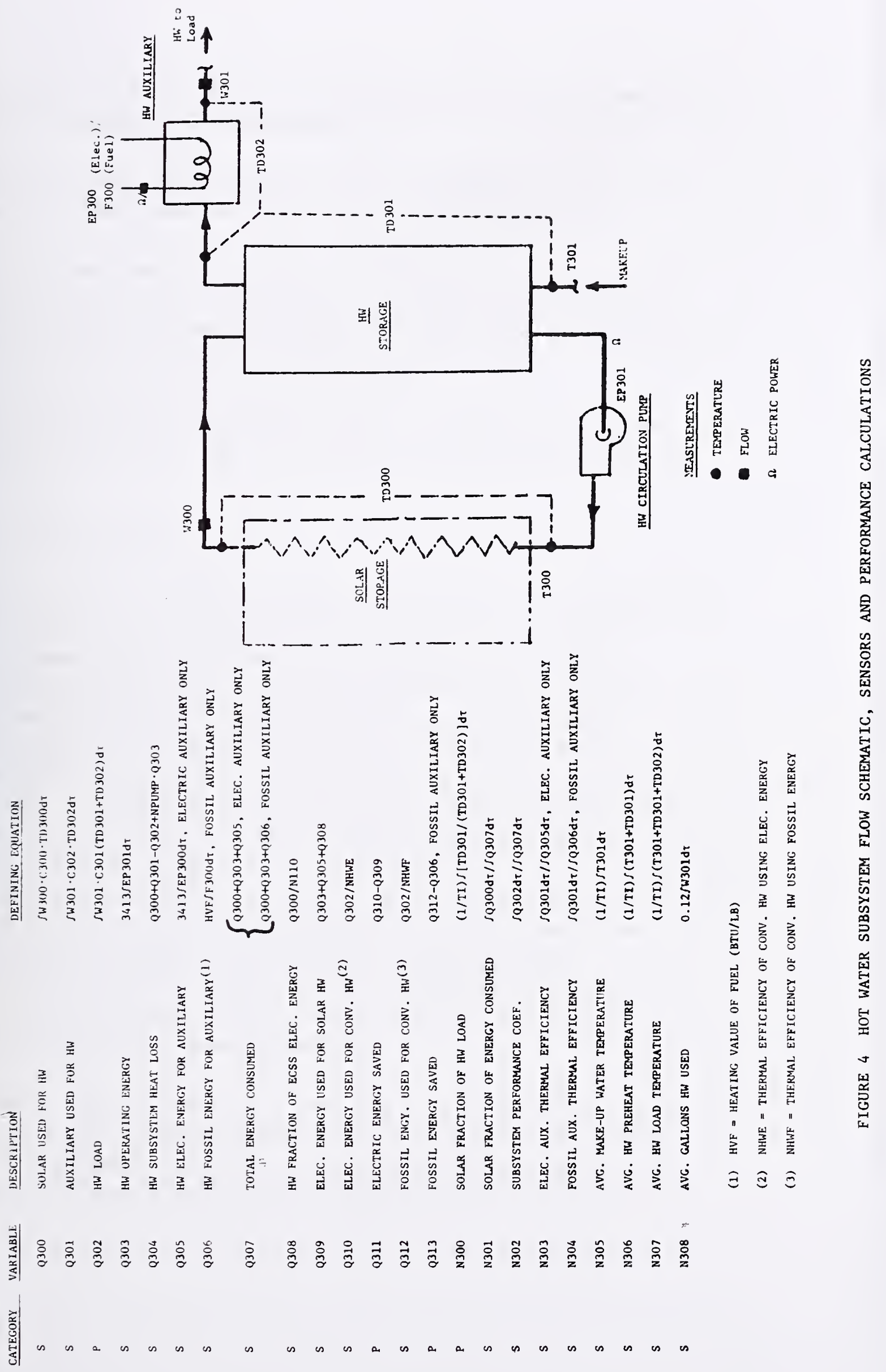



Insufficient, auxiliary energy is added either electrically or by combustion of fuel to provide the desired HW temperature.

Temperature sensors are located at the HW heat exchanger inlet and at the makeup to the HW storage tank and across the auxiliary heating unit.

Liquid flow measurements are required for the HW circulation loop and for the $\mathrm{HW}$ flow to the load. Operating power measurements include electrical energy consumed by the pump and controls. Electrical power or fuel flow rate must be measured depending on the auxiliary energy source.

Figure 4 also lists the performance calculations required for the HW subsystem. A further discussion of the primary performance factors is given in section 6 .

4.3 Space Heating Subsystem

Figure 5 shows the flow schematic, instrumentation requirements and performance calculations for two alternate space heating subsystems using a liquid heat transport medium. In SYSTEM A, an all-electric heating system, a liquid-to-air heat exchanger is located in the air duct as the primary solar heating component, a liquid-to-air heat pump is the secondary solar-heating "component and an electric resistance heating coil is the backup auxiliary component which is used when the solar energy storage is depleted. In SYSTEM B, an all fossil fuel heating system, solar heated liquid is passed through a liquid-to-air heat exchanger located in a conventional fan-coil heating unit. Auxiliary energy is provided in the heat transport loop by a conventional gas or oil fired furnace to supplement the solar energy source during peak demand periods or when insufficient solar energy is available. In both systems the building supply air is heated as it flows across the various heat exchangers located in the air duct and this increase in sensible energy represents the amount of heat provided to the space heating load.

In SYSTEM A, four distinct modes of operation are considered, which are numbered in order of increasing electrical energy consumption and therefore decreasing preference. In Mode 1, when the temperature of the storage medium is sufficiently high to carry the building heating load, the two-way 

SYSTEM A

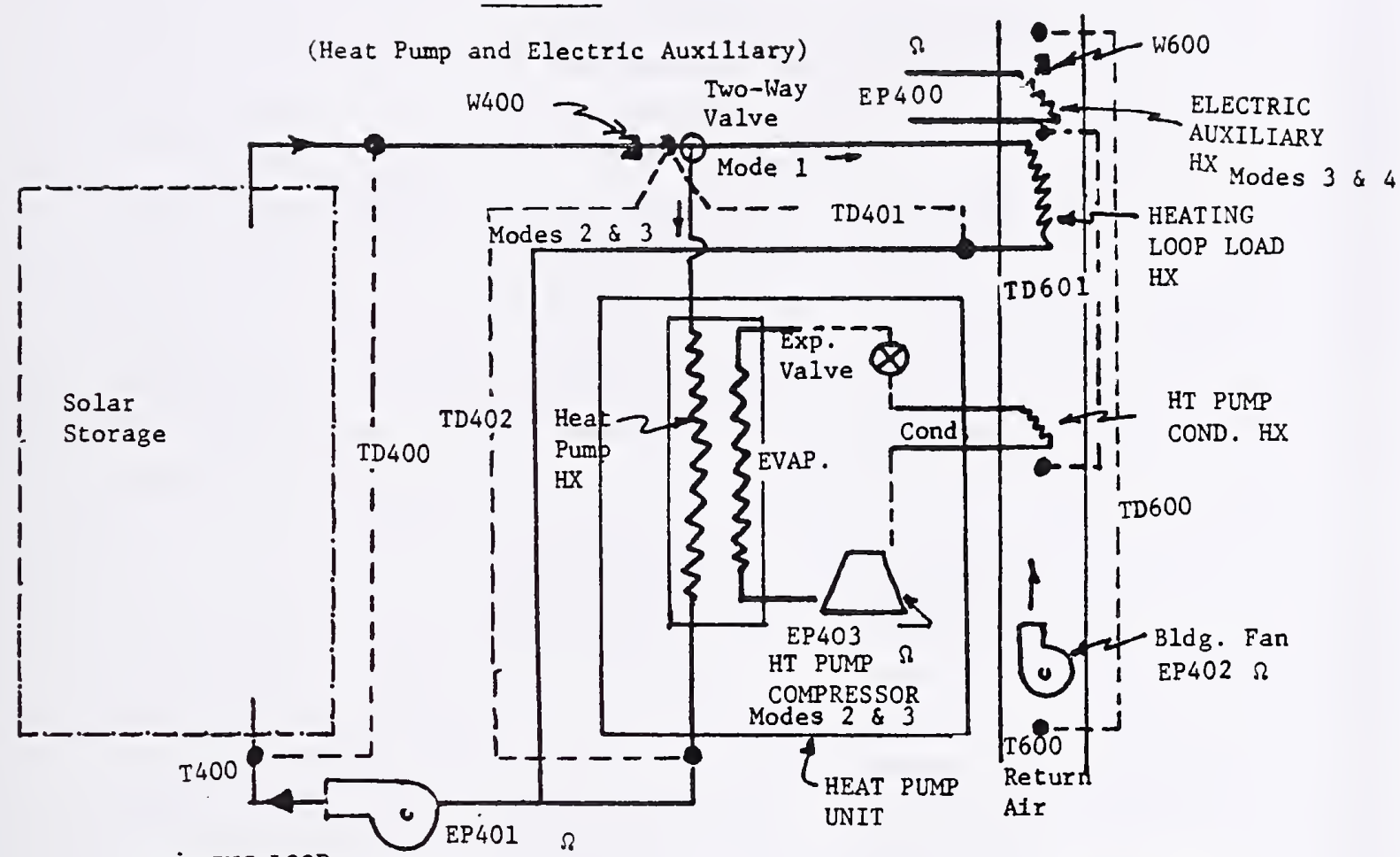

HEATING LOOP

CIRCULATION PUMP

SYSTEM B

MEASUREMENTS

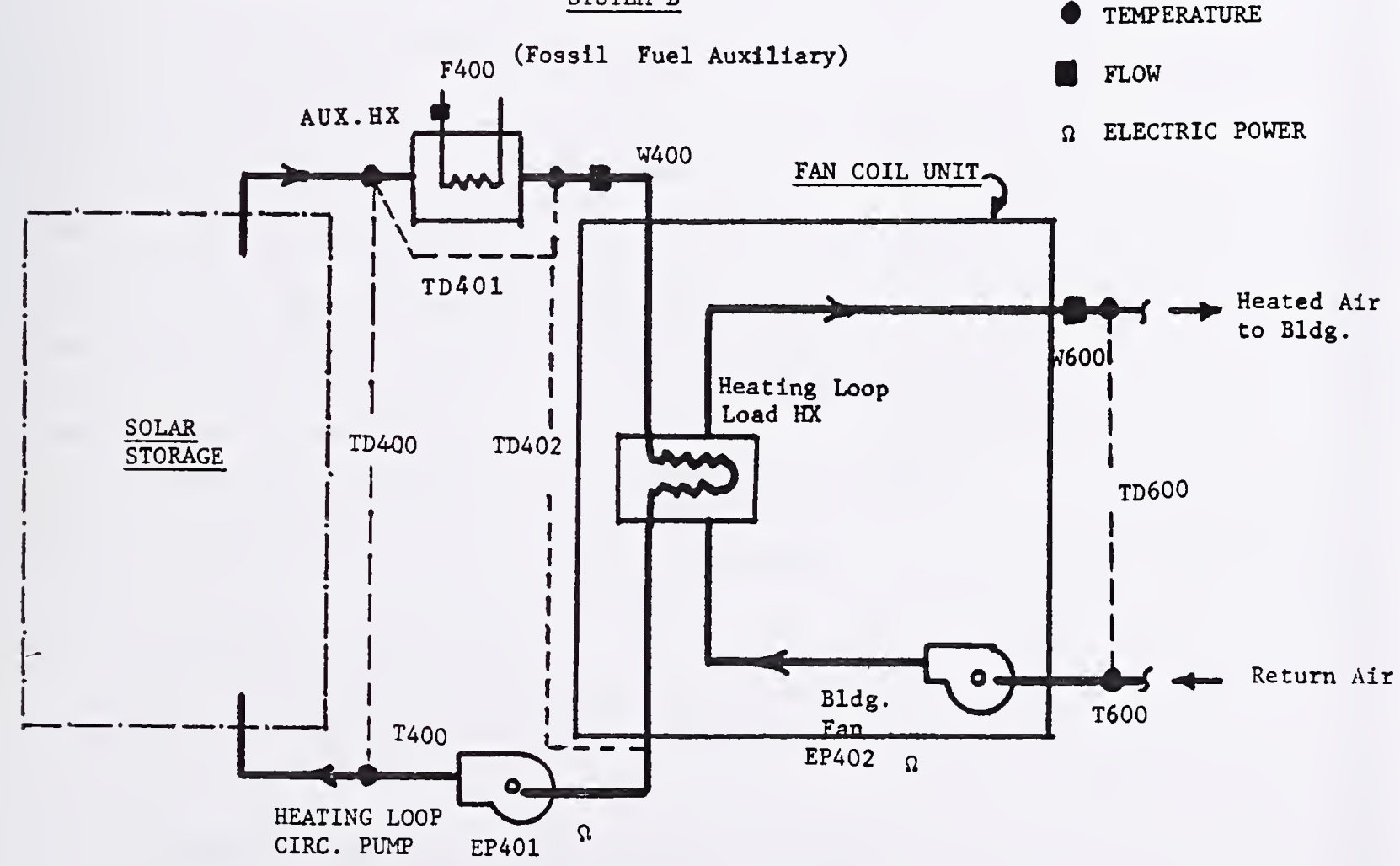

FIGURE 5 SPACE HEATING SUBSYSTEM FLOW SCHEMATICS AND SENSORS 



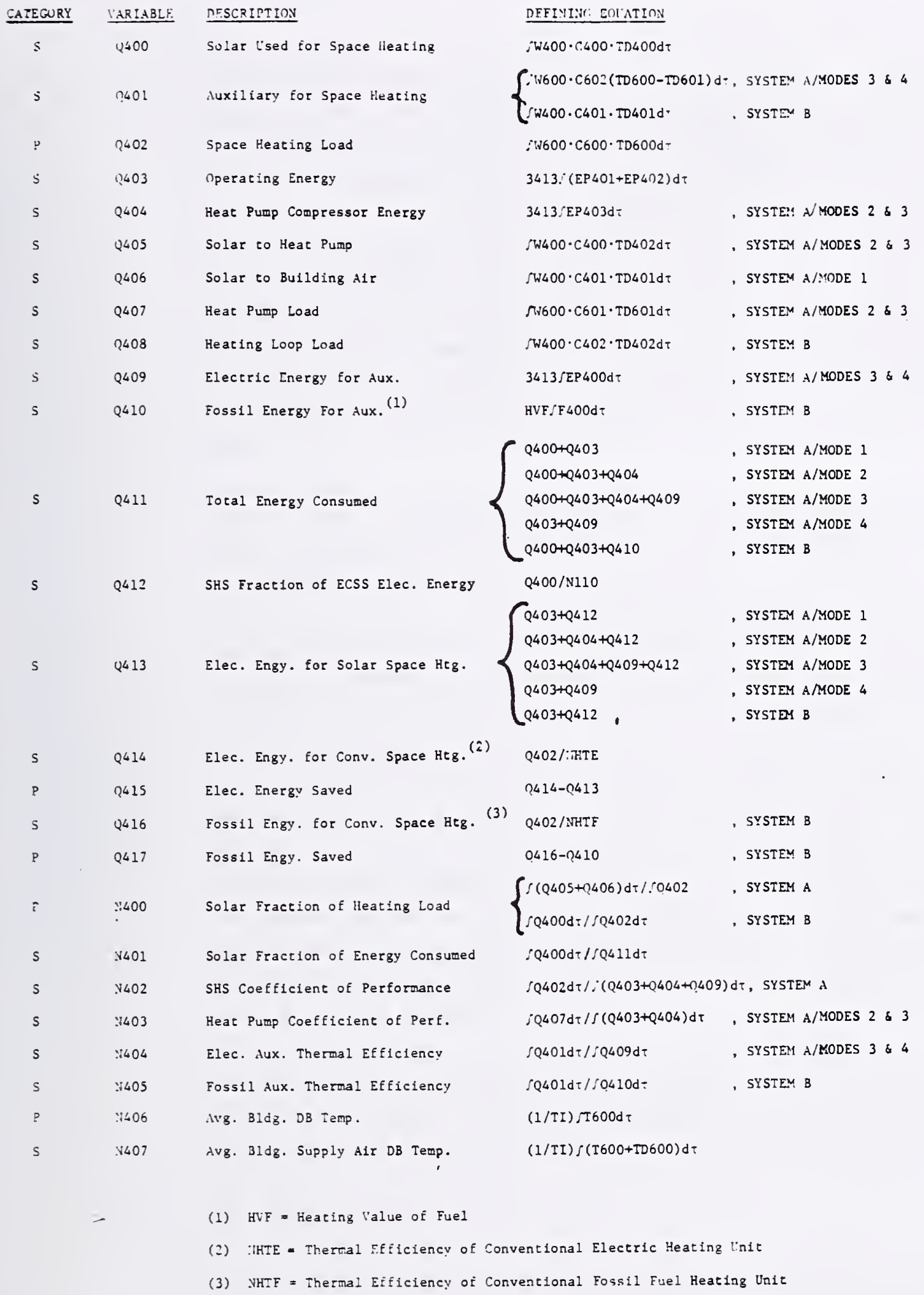



valve directs the flow of solar heated liquid to the heating loop load heat exchanger. If the temperature of the solar heated liquid is insufficient to carry the building load, Mode 2 operation is initiated in which the two-way valve diverts the heating loop flow to the heat pump's evaporator heat exchanger and the heat pump is turned on. This mode uses solar energy indirectly in that the solar heated liquid is used to increase the heat pump's coefficient of Performance (COP) and thus reduce the consumption of electrical energy required to carry the building heating load. In Mode 3, the heat pump's heating capacity is augmented by the electric resistance heaters in the air duct and in Mode 4 the heat pump and heating loop circulation pump are off and the entire building heating load is carried by the electric resistance heaters.

Figure 5 shows the measurements required for the space heating subsystem. Absolute temperature sensors are located at the heating loop inlet to the solar storage subsystem and at the building return air duct inlet to the heat pump or fan coil unit. Differential temperature sensors are located in the heating loop across the solar storage subsystem, across the heat pump heat exchanger In SYSTEM $A$ and across the heating loop load heat exchanger. A differential temperature sensor is required in the bullding air loop to sense the dry-bulb temperature difference between the bullding alr supply and return and in SYSTEM A to sense temperature difference across the combined heat pump condensor heat exchanger and the electric auxiliary coll. A liquid nuass flow sensor is located in the heating loop and an alr mass flow sensor is located in the building air flow circuit. Operating power measurements are raquired for the heating loop pump, bullding fan and controls for both systems, and for the heat pump compressor of SYSTEM A. Auxiliary energy consumption, using an electric meter for SYSTEM $A$ and a fuel flow sensor for SYSTEM $B$ are also required.

The performance calculations required for the space heating subsystem are also listed in Figure 5. The primary performance evaluation factors used to characterize the operation of the subsystem are further described in Section 6 .

- 4.4 Space Cooling Subsystem

Figure 6 shows the flow schematic, instrumentation requirements and detailed performance calculations for the space cooling subsystem using a thermal energy actuated absorption chiller as the cooling component. In operation, the solar heated liquid from storage is circulated through the absorption chiller's generator by way of the auxiliary heat exchanger and this provides the required thermal energy source. 



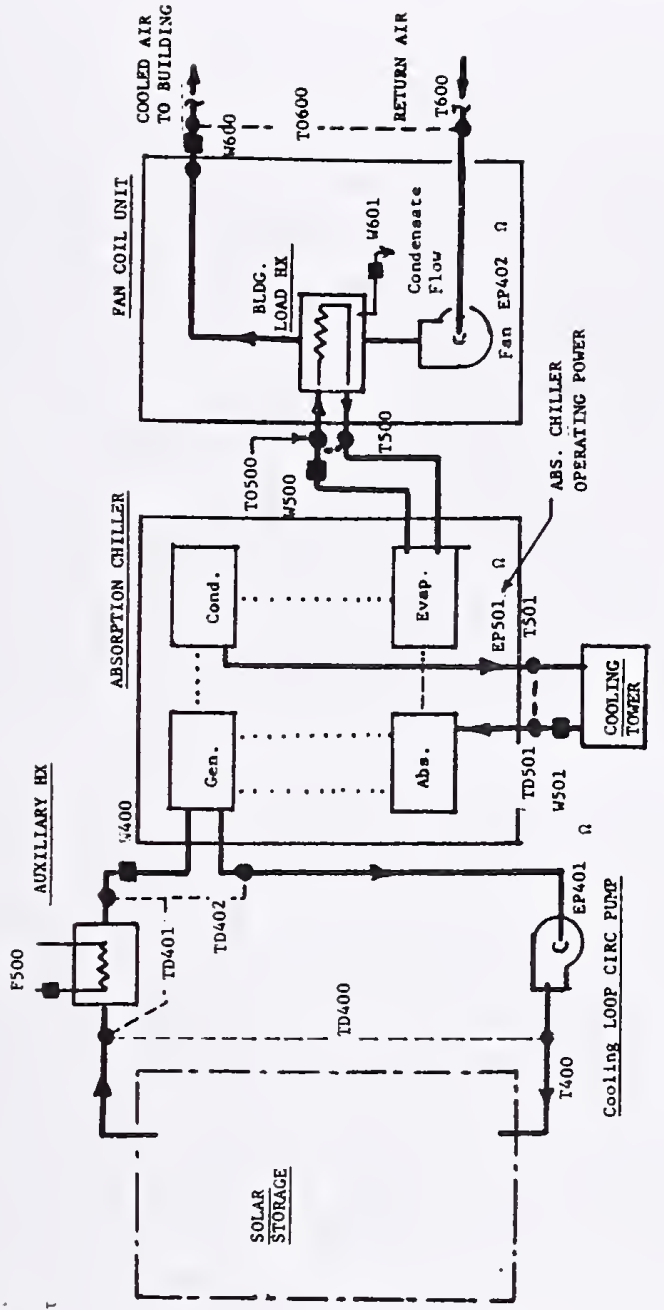

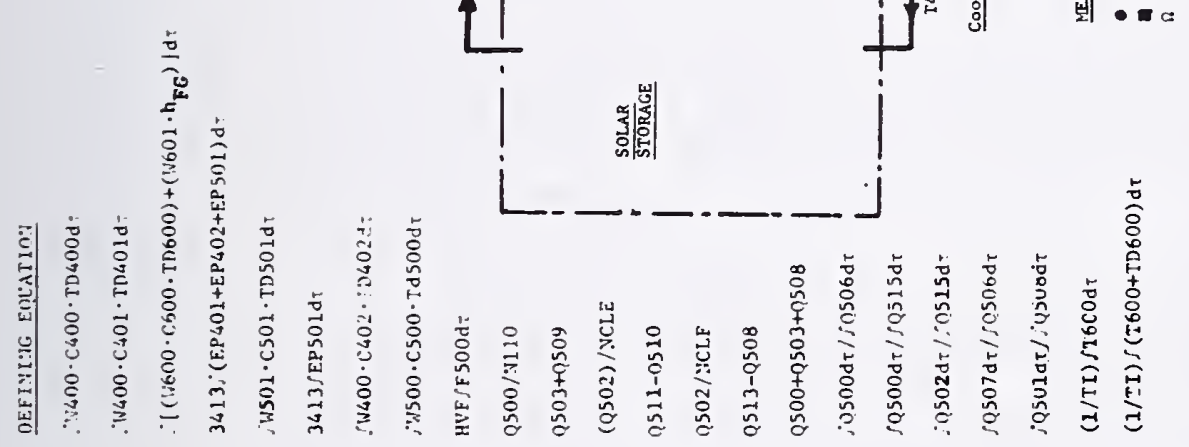

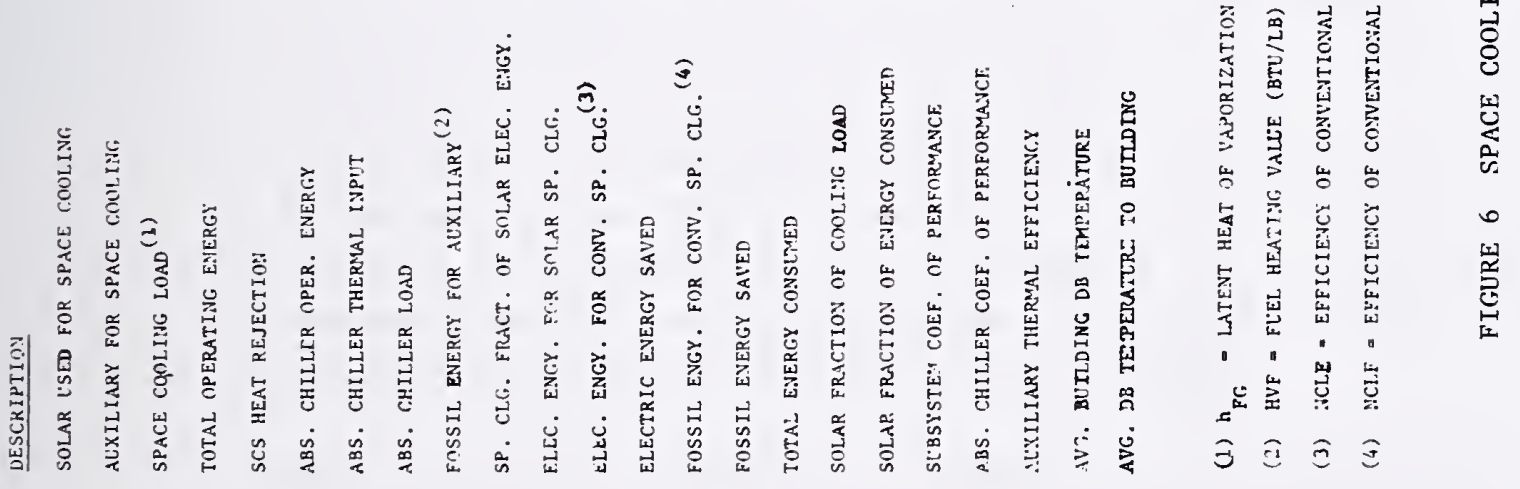
然

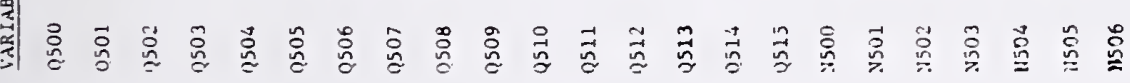
递 

The absorption chiller's useful thermal output conslsts of the energy indirectly removed from the building air using an intermediate heat transfer fluid (chilled water or brine) which is pumped through the evaporator. The heat refection required by the absorption thermodynamic cycle is provided by another intermediate heat transfer fluid (water) which absorbs heat in passing through the absorber and the condensor sections and then rejects this heat to ambient air via a cooling tower. The energy removed from the bullding alr flowing over the load heat exchanger represents the space cooling load. Since most space cooling applications include both sensible cooling and dehumidification processes, it is important to account for the removal of water vapor from the building air in determining the building's cooling load.

Figure 6 shows the measurements required to fully evaluate performance of a solar assisted space cooling subsystem. The temperature, flow and power sensors for the heating loop and fan coil unit are identical to those previously described for the system B configuration space heating subsystem. Additional temperature and flow sensors are required to measure absorption chiller heat rejection and building air dehumidification. Bullding air dehumidification is determined using a modified rain gauge to determine the rate of condensation at the building load heat exchanger. An additional power sensor is required to measure the operating power of the various pumps, fans and controls used in the absorption chiller.

In measuring wet-bulb temperature (or alternatively measuring relative humidity) it is recognized that the relative high expense and reduced accuracy of the commercially available sensors may not be justifled, particularly for the residential portion of the demonstration program. Alternatively, the bullding cooling load could be defined as the energy added to the chilled water circulating between the absorption chiller and load heat exchanger at the load heat exchanger. This approach does not account for the energy gains in the fan coll unit but does eliminate the requirement for the condensate measurement. Measurement of the alternative building cooling load at the absorption chiller requires coolant mass flow and inlet temperature to the load heat exchanger and temperature difference across the load heat exchanger.

Figure 6 also lists the performance calculations required for the space cooling subsystem and the primary items which characterize the operation of the subsystem and are reported in the overall performance summary. These 1 tems are further discussed in Section 6 . 

The influence of the building envelope materials and construction, particularly the area and location of windows, on bullding heating and cooling loads are well established. The application of windows, walls, roof sections with sky lights and roof ponds as part of the architectural design to utflize solar energy as a significant fraction of the tntal 'oad is commonly referred to as a passive solar energy system. Additional requisites for a building design using windows to qualify and be evaluated as an alternative energy source have been stated in reference [2] as (1) operable insulating shutters or other devices which, when drawn or closed, shall cause the window area to keep maximum outward heat flows below a selected value, and (2) the window areas are shaded to otherwise protected from the direct solar rays during the cooling season.

Generally the same functional elements such as the collector, storage and energy conversion subsystems exist in passive systems but physical distinction between these elements and the building elements is not always possible. Direct measurement of the thermal energy flows will probably not be possible because of the dependence on natural heat transport phenomena such as free convection, radiation and evaporation. Therefore, the heat balance approach previously described for active systems will have to be greatly modified for passive system evaluation.

In general, the only direct measurements practical will be with temperature sensors, heat flow meters, scanning radiometers and pyranometers. The performance evaluation will rely upon measurements of the auxiliary fuel usage and the interfor comfort level. Determination of the energy saved will be obtained from load calculations for simflar buildings without the solar energy system or, when possible, measured energy consumption in an adjacent similar structure without a solar energy system.

The following section describes the energy flow, instrumentation requirements and performance evaluation for two alternate designs assumed for illustrating the use of passive solar energy for space heating and cooling. The systems are considered passive because no auxiliary energy is required for the transfer of thermal energy between the collecting and storage elements and the building load. Figure 7 illustrates conceptually the two passive solar energy systems and indicates the required instrumentation for performance evaluation.

One design consists of a water pond with a movable insulation cover that is thermally integrated into the building structure. The other design consists of a wall solar collector with a movable insulating device and thermal storage 



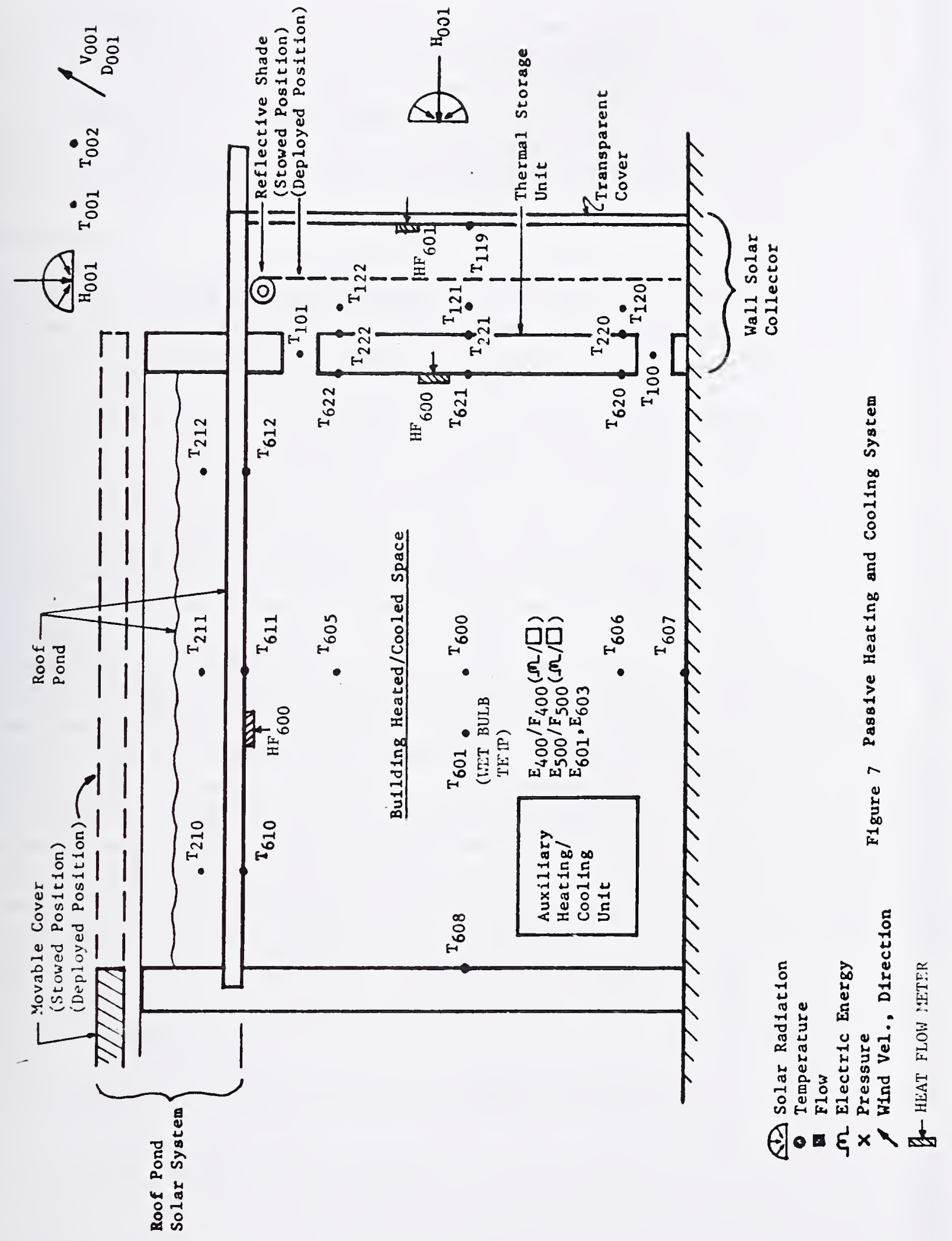



that is also thermally integrated into the building structure. These two designs are considered representative of current passive technology but many other innovative architectural approaches have been built for private use and their evaluation is in a relatively primitive stage. It is apparent that the building solar heat gain, thermal resistance, thermal mass and thermal time constant are the basic parameters governing the bullding thermal performance.

5.1 Roof Water Ponds

Figure 7 shows the daytime position of the movable insulating cover during the heating season. In operation, the roof pond is uncovered during the heating season daytime, thereby enabling solar energy to be stored by raising the internal energy of the water in the roof pond. At night, the pond is covered to reduce heat losses and enable the stored energy to be used for space heating by free convection and radiation from the celling. During the cooling season, at night the roof panel is uncovered and the pond rejects heat by radiation to the night sky and by evaporation. During the daytime, the roof panel is covered to prevent absorption of solar energy. Space cooling is provided during the day because the cool water in the roof panel can absorb the heat that is released in the space and transferred through the walls and fenestrations.

Roof water ponds can be evaluated in the same manner as conventional solar collectors. In order to do this, the overall heat transfer coefficient (UA) must be known as a function of the temperature difference between the roof average pond water $\left(T_{w}\right)$ and the average building air $\left(T_{a}\right)$. This relationship can be quantitatively established by heating the roof pond with solar energy until a maximum temperature for the day is obtained at which time the roof water pond is covered with the insulated panels. The rate at which the water temperature decreases times the water mass $(m)$ and specific heat $\left(c_{p}\right)$ gives the rate of heat transfer to the building, assuming that the heat loss through the insulated panels is either negligible or can be calculated. Once this quantitative relationship is established, the useful heat delivered by the water roof pond to the building can be calculated knowing the value of the aforementioned temperature difference.

The solar energy collected between time $\tau_{1}$ and $\tau_{2}$, Q100 is calculated by:

$$
Q 100=m \cdot c_{p}\left[T_{w}\left(\tau_{2}\right)-T_{w}\left(\tau_{1}\right)\right]+\int_{\tau_{1}}^{\tau_{2}} U A\left(T_{w}-T_{a}\right) d \tau
$$



Where UA is the overall heat transfer coeffictent between the pond and the ceiling, and $T_{W}\left(\tau_{2}\right)-T_{W}\left(\tau_{1}\right)$ is the increase in the average pond temperature during the selected time intervals when the pond is uncovered. From a practical standpoint, daily collector efficiency is the minimum time to obtain reasonable accuracy for performance evaluation. The efficiency can be expressed by:

$$
\mathrm{N} 100=\int_{\tau_{1}}^{\tau_{2}} \mathrm{Q} 100 \mathrm{~d} \tau / \int_{\tau_{1}}^{\tau_{2}} \mathrm{Q} 001 \mathrm{~d} \tau
$$

where the energy collected and the incident solar radiation quantities are summed each day when the pond is uncovered.

The rate at which heat is removed from a building during the summer can be determined in a similar manner. Just before sunrise, the roof water pond is covered with the insulated roof panels. The rate of water temperature increase times the water mass and specific heat product gives the rate at which heat is removed from the building (if the heat transfer through the insulated roof panels is either negligible or can be calculated) as a function of the temperature difference between the water and building air.

It is imperative that the internal afr temperature be carefully recorded because performance will depend significantly on the temperature excursions permitted. In addition, it is extremely important that the roof pond water depth also be carefully recorded because it will also affect performance.

The fraction of the annual heating and cooling load furnished by a roof water pond is strongly dependent on the temperature excursions permitted before auxiliary energy is used. Therefore, internal temperature is required for space heating and cooling and relative humidity measurements are needed for the evaluation of space cooling. The electrical power expended for humidity control must be measured also.

Measurements of the fossil or electrical energy used for auxiliary purposes (E400/F400 and E500/F500) are used to determine the total auxiliary energy, Q401 (heating) and Q501 (cooling). The heating or cooling load, Q402 or Q502 respectively, are obtained by adding the solar and auxiliary contributions as follows:

$$
\text { Q402 }=\int_{\tau_{1}}^{\tau_{2} 2}\left[U A\left(T_{w}-T_{a}\right) d \tau+Q 401\right.
$$





$$
Q 502=\int_{\tau_{1}}^{\tau_{2}}\left[\mathrm{UA}\left(\mathrm{T}_{\mathrm{a}}-\mathrm{T}_{\mathrm{W}}\right) \mathrm{d} \tau+Q 501\right.
$$

Because of the bullding modification to accommodate the roof pond, energy saved calculations will require analytical prediction of the building load for conventional design and construction.

Wall Collector

The operation of the wall solar collector is shown in Figure 7 during the heating season for sunny days; the insulating device is removed thereby exposing the high thermal capacity wall to solar radiation. At night, the deployed insulating device and transparent cover reduce heat losses to ambient and the heat stored in the wall during the day is transferred by convection and radiation to the space for heating. The wall collector is primarily a heating device so that the useful output during the cooling season is limited to the ventilation capability of the system. To limit solar heat gains during the cooling season, the insulating device must be deployed and the overhanging roof designed to provide shading from direct solar radiation.

For the generalized system schematic in Figure 7 the possible heat flows are the solar input, I001, heat losses from the wall (a function of the wall or window temperatures T119, ambient temperature T001, wind characteristics v001 and D001, and radiation exchange with the exterior surrounding) and the heat delivered or removed from the building heated space.

5.3 Performance Evaluation

Figure 7 shows the sensors utilized for performance evaluation of the passive type solar systems. Although temperature sensors are Indicated for the energy storage elements, heat flow meters would also be appropriate. Because of the passive nature of the solar system, air flow measurements are probably impractical and energy transfer and storage must be determined from the measured temperatures and a knowledge of the thermal characteristics of the bullding. Thus, performance analysis of the passive solar system must rely primarily on transient thermal analysis of the integrated bullding and solar system, considering the outdoor climatic conditions and the energy release within the bullding. It should be noted that the exact number and location of the temperature/ heat flow sensors are very dependent on the design of the particular instrumentation deemed necessary for passive system evaluation.

The heat transfer calculations for room temperature predictions are similar to 

the building heating/cooling load calculations performed by avallable computer programs. However, the temperature calculations are more complex because they require exact heat balances considering the room air, surrounding walls and infiltration of the outdoor air. Since the major variable in passive system performance is the hourly room temperature, it is necessary to account for the transient heat conduction and thermal storage of the building and internal mass.

Determination of the temperature and humidity in non-air conditioned rooms responding to randomly fluctuating outdoor climatic conditions can be determined using hourly simulations of heat gain and sturage obtained with standard algorithams published by ASHRAE [3].

A computer program employing these algorithms is avallable [4]. The calculation sequence as depicted in Figure 8 , requires detailed data input for the building, weather and operating schedule. Bullding data includes geometric characterization of the construction and materials used for the exterior envelope, interior partitions, cellings and floors and their associated thermophysical properties. Weather data is provided in the form of a National Weather Service Tape or provided as measured data including wet and dry bulb temperature, wind, and solar radiation. The operating schedule is used to determine energy release profiles for the appliances and lights and metabolic heat release by the occupants.

Normal solar radiation inputs corrected for local cloud cover, collector tilt angle and shading can be calculated for conventional bullding construction heat gain or for solar modifled construction using roof ponds and window collectors. Routines to calculate the transmission, reflection and absorption for direct and diffuse solar radiation are used to calculate the solar heat gain through fenestrations. The thermal response of the varfous building structural components and furnishings are calculated using conduction, radiation and convection heat transfer routines to provide the necessary inputs to the room temperature calculation (RMTMP) [4]. This subroutine compares the prescribed room temperature with the calculated temperature, and variances beyond the prescribed limits result in calculation of the heating or cooling loads to satisfy the temperature. 



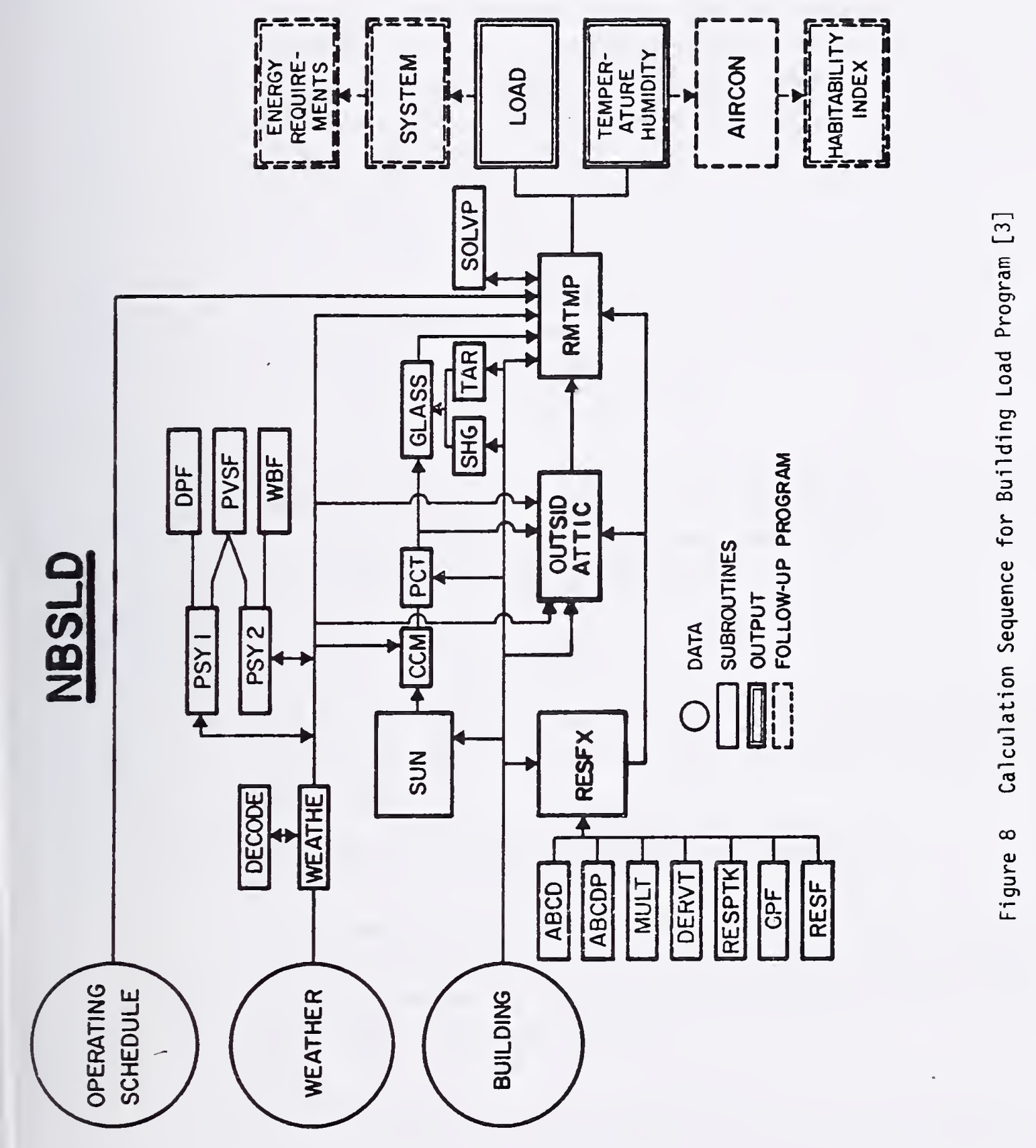



As indicated in Figures 3 to 6 , numerous calculations are performed for the elements comprising a system, subsystem or for the individual components in the solar assisted heating and cooling system. As previously described, performance evaluation factors are classified into three categories, primary, secondary and special. The primary performance evaluation factors are 1 isted in Table 1 and consist of those evaluation factors that summarize the performance of each subsystem, the solar energy system, the building and climate and the utilization of the solar energy system by the occupant. The secondary performance evaluation factors include the detailed performance of the solar collectors, storage tanks, heat exchangers and other solar energy components as well as the components of the conventional HVAC equipment used such as hot water heaters, furnaces, heat pumps and other energy conversion equipment. Special evaluation factors include those calculations performed using data from certain optional sensors such as the diffuse pyramometer or the collector absorber panel temperature.

This section will describe each term of the primary system performance evaluation summary to clearly define the terminology and calculation method. In addition, some of the more important terms of the secondary and special performance calculations are described.

6.1 Performance Factors - General

Two types of performance factors are indicated in Table 1; a thermal energy quantity defined by the letter "Q", and a performance index defined by the letter "N".

\subsubsection{Performance Factors Based on Thermal Energy Quantity}

In the evaluation of the net thermal energy flow into or out of a subsystem, the general expression for energy addition to a fluid flowing through a subsystem or component is determined by integrating the measured instantaneous rate of heat addition as follows:

$$
\begin{aligned}
& Q=\int_{\tau_{1}}^{{ }^{2}} \dot{\mathrm{mc}}_{\mathrm{p}}\left(\mathrm{T}_{\text {out }}-\mathrm{T}_{\mathrm{in}}\right) \mathrm{d} \tau \\
& \text { where } \dot{\mathrm{m}} \text { is the fluid mass flow rate in } \mathrm{LB} / \mathrm{HR}, \\
& c_{p} \text { is the fluid specific heat at constant pressure in } \mathrm{BTU} / \mathrm{LB}{ }^{\circ} \mathrm{F},
\end{aligned}
$$



$T_{\text {out }}-T_{\text {in }}$ is the fluid temperatur $=-5 \equiv$ is the subsystem or component. in ${ }^{\circ} \mathrm{F}$ and

$\tau_{1}, \tau_{2}$ are the inftial and final $=z-z=z$ tie integration period.

In Exeral, both $T_{\text {out }}$ and $T_{\text {In }}$ are functions $=\equiv \pm=i m$, $\dot{I}$ is a function of both tire and temperature and $c_{p}$ is a function of =0 =ature: therefore, evaluation of Eruation (6.1) usually involves a numeric : $=$ =egration procedure in which the =ozal time interval is broken down into $\equiv-1=$ sma:- so that during the subinterval each va: $=-\equiv$ in the integrand can be co=E-_=Ied constant.

Idea-- continuous data measurement is desiz三. :ovever, in the actual data

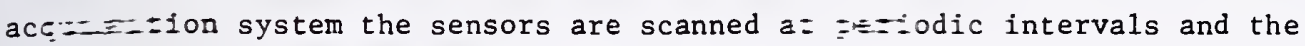
ins -aneous measured values are used to eva $=z=\sum$ equation (6.1). Changes in $=\equiv$ variables between data scans are not $\Sigma \equiv=\equiv=-$ ined and errors are introduced int: = $=$ E calculated results. Section 7 desc $=2 \vdots \equiv \equiv$ these errors due to data sam: $=-\equiv$ and presents the results of a study $=\equiv \equiv$ mpling errors based on dat $\equiv=x$ one of the solar demonstration hous $\equiv \equiv \equiv \pm$ the Colorado State Uni $= \pm[5$ [5].

In $\equiv=a 1$, for all thermal energy quantities $=-\bar{s}$ smallest integration pertod

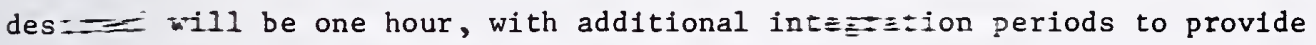
cumi- = ve daily, monthly, and seasonal or ar:=E- data.

\section{.1.2 Per $= \pm$ =ance Indices}

In $=$ Evaluation of a performance index whic jepends on the ratio of

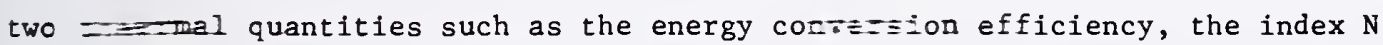
is

$$
S=\frac{\int_{\tau_{1}}^{\tau_{2}} Q_{1} d \tau}{\int_{\tau_{1}}^{\tau_{2}} Q_{2} d \tau}
$$

In Equa - - $=(\vdots .1)$. For meaningful results the $i=z \equiv \equiv$ tion times must be sufficiently long: $=-=0$ ly to preclude division by zero, $\equiv= \pm=0$ assure that a true average value = ozzained. Thus, the performance inceI = =lculations involving the ratio of the- energy quantities may require eval z=zon on a daily, monthly, or annua- asis using hourly values of the therme- Dergy quantity. 

Other performance indices such as average temperature are determined by integrating the measured temperature and dividing by the integration period as follows:

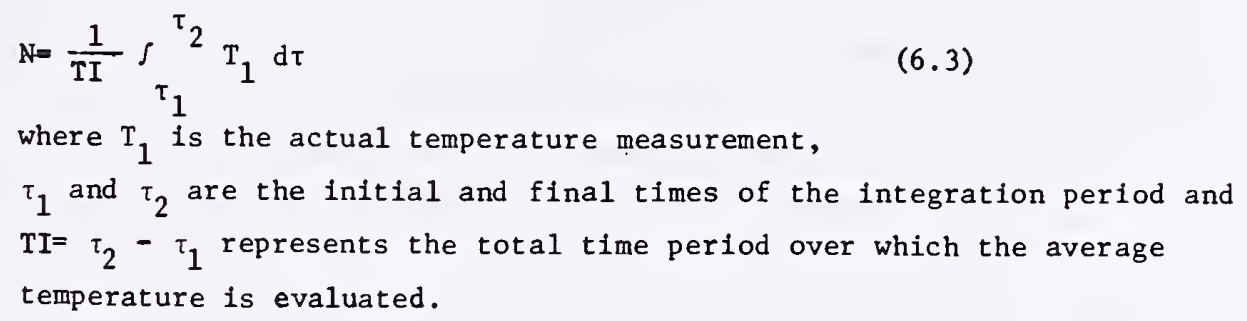

The minimum integration period varies depending on the particular temperature. For example, outdoor dry bulb temperature would be desired for hourly, daily and monthly periods whereas storage tank ambient temperature might be evaluated on a monthly basis.

\subsection{Energy Saved - General}

Since the energy sources for both conventional and solar assisted heating and cooling include different energy types such as gas, oil or electrical, it is important to distinguish between at least the fossil sources and the electrical sources. It is conceivable that when a conventional system is replaced with a solar system, the solar system could require more electrical energy to run (due to operating power for the solar pumps, fans or controls) than the conventional system; however, the solar system should significantly reduce the consumption of fossil energy. Due to the difference in energy costs in various parts of the country, the same solar system and same type building in different locations may have wide variance in the cost effectiveness or payback period.

The basic approach to determining the energy saved is to first measure the total electrical energy and the total fossil fuel energy consumed by the solar assisted system to provide a given building energy load at a given occupant utilization level. An assessment of a non-solar system must then be made to determine the type of equipment that would be provided if there were no solar equipment.

Since available performance data of energy conversion equipment are usually based on measurements taken at a design condition for steady state equipment operation, the effects of off-design operation and duty cycling on equipment performance must be estimated to improve the accuracy of the predicted energy consumption. Alternatively, equipment performance based on seasonal efficiency rather than design point efficiency should be used. The electrical energy 

and fossil energy consumption of the non-solar system must then be calculated assuming some equipment performance coefficients and operating efficiencies based on the same building load and occupancy utilization that was measured for the solar system. The energy saved is then the difference between the calculated value of energy consumed by the non-solar system and the measured value of energy consumed by the solar system. In reporting these results, the assumed non-solar system must be described as well as the assumed performance characteristics and efficiencies.

\subsection{Climatic Primary Performance Factors}

\subsubsection{Total Solar Energy Incident (Q001,Q002*)}

Total incident solar radiation is measured by a sensor mounted in the plane of the collector array on either a continuously integrated or frequently sampled basis. Data are recorded at specified intervals for use in the calculation of collector and system efficiencies. For those solar energy systems having a significant dependence on direct solar radiation (such as concentrating collectors), diffuse insolation will also be measured independently with a separate sensor located in the plane of the collector array and the direct component of incident solar radiation is determined by subtraction of diffuse from the total incident solar radiation. The total and direct integrated values of incident solar radiation are calculated by integrating the instantaneous values as follows:

$$
\begin{aligned}
& \mathrm{Q} 001=\int_{\tau_{1}}^{\tau_{2}} \quad \text { I001 d } \tau \\
& \text { Q002* }=\int_{{ }^{\tau} 1}^{\tau_{2}}(\text { I001- I002) } d \tau
\end{aligned}
$$

Where I001 and I002 are the instantaneous values of incident total solar radiation and diffuse solar radiation respectively, and $\tau_{1}, \tau_{2}$ are the integrating time intervals.

Q002* is not identified as a primary performance factor in Table 1 however, its importance for certain solar collectors warrants inclusion as a special measurement for some active and passive solar energy systems. 



\subsubsection{Average Ambient Dry Bulb Temperature (N113)}

Average ambient dry bulb temperature is a significant parameter in determination of system performance and comparison of alternate solar energy systems in different climatic regions. It is obtained by integration of the instantaneous ambient dry bulb temperatures as follows:

$$
\mathrm{N} 113=\frac{1}{\mathrm{TI}} \int_{\tau_{1}}^{\tau_{2}} \mathrm{~T} 001 \mathrm{~d} \tau
$$

where T001 is the instantaneous dry bulb temperature, and TI is the integration period $\tau_{2}-\tau_{1}$.

N113 should be obtained for the hourly, daily and monthly data summaries.

6.4 Energy Collection and Storage Subsystem (ECSS) Primary Performance Factors

The primary performance factors are based on the ECSS shown in Figure 3.

\subsubsection{Solar Energy Collected (Q100)}

The amount of solar energy collected in a given time period is obtained by evaluating Equation 6.1 using the appropriate sensors in the ECSS as follows:

$$
\text { Q100 }=\frac{1}{\mathrm{~A}_{\mathrm{C}}} \quad \int_{\tau_{1}}^{\tau_{2}} \mathrm{W100} \cdot \mathrm{C} 100 \cdot \mathrm{TD} 100 \mathrm{~d} \tau
$$

Where $A_{C}$ is the total collector area defined by the outside dimensions of the collector array, *

W100 $=$ Collector array fluid mass flow rate, C100 = Specific heat of collector fluid, TD100 = Fluid temperature increase across the collector array.

Additional data may be required to correct the fluid specific heat for temperature, concentration, etc.

\subsubsection{Total Solar Energy Utilized (Q300, Q400, Q500, Q203)}

For the demonstration program, solar energy is used in as many as three applications; i.e., hot water heating, space heating, and space cooling. For each application,

\footnotetext{
*Proposed Solar collector test procedure [6] rates flat plate collector performance on transparant frontal area instead of total area, therefore, thermal comparison of result will require adjustment by appropriate erea ratio.
} 

Equation (6.1) is evaluated using the appropriate measurements in the HW loop and in the heating or cooling loop. Since some of the measurements used for the heating and cooling calculations are the same, additional information must be supplied (such as the operating status of the absorption unit) to determine whether the calculation pertains to heating or cooling. The following equations are used to calculate each component for the ECSS using a liquid heat transfer medium (SYSTEM A in Figure 3):

6.4.2.1 Solar Used for Hot Water

$$
\mathrm{Q} 300=\int_{\tau_{1}}^{\tau_{2}} \mathrm{W3} 00 \cdot \mathrm{C} 301 \cdot \mathrm{TD} 301 \mathrm{~d} \tau
$$

6.4.2.2 Solar Used for Space Heating

$$
\mathrm{Q} 400=\int_{\tau_{1}}^{\tau_{2}} \mathrm{W400} \cdot \mathrm{C} 400 \cdot \mathrm{TD} 400 \mathrm{~d} \tau
$$

6.4.2.3 Solar Used for Space Cooling

$$
Q 500=\int_{\tau_{1}}^{\tau_{2}} W 400 \cdot \mathrm{C} 400 \cdot \mathrm{TD} 400 \mathrm{~d} \tau
$$

\subsubsection{Total Solar Energy Utilized (Liquid Heat Transfer Medium)}

The total solar energy utilized is the sum of the solar energy used in each application and is given by:

$$
\mathrm{Q} 203=\mathrm{Q} 300+\mathrm{Q} 400+\mathrm{Q} 500
$$

\subsubsection{Total Solar Energy Utilized (Air Heat Transfer Medium)}

For the ECSS using air as the heat transfer medium (SYSTEM B in Figure 3 ). the total solar energy utilized is given by:

$$
\mathrm{Q} 203=\int_{\tau_{1}}^{\tau_{2}} \mathrm{W400} \cdot \mathrm{C} 401 \cdot \mathrm{TD} 401 \mathrm{~d} \tau
$$

\subsubsection{ECSS Conversion Efficiency (N111)}

The ratio of total solar energy actually utilized to the total solar energy incident on the collector array is termed the solar subsystem conversion 

efficlency and is a measure of the abllity of the ECSS to convert incident solar radiation into useful thermal energy. ECSS conversion efficiency is calculated by:

$$
\mathrm{N} 111=\int_{\tau_{1}}^{\tau_{2}} \mathrm{Q} 203 \mathrm{~d} \tau / \mathrm{A}_{\mathrm{c}} \int_{\tau_{1}}^{\tau_{2}} \mathrm{Q} 001 \mathrm{~d} \tau
$$

Because of the solar energy storage time dependence, ECSS conversion efficiency (N111) should be determined only for the monthly. seasonal and annual data summary.

\subsection{Hot. Water Subsystem (HWS) Primary Performance Factors}

\subsubsection{Hot Water Load (Q302)}

The total thermal energy required for heating HW from the inlet (makeup) to delivery temperature is given by:

$$
\mathrm{Q} 302=\int_{\tau_{1}}^{\tau_{2}} \mathrm{~W} 301 \cdot \mathrm{C} 301 \cdot(\mathrm{TD} 301+\mathrm{TD} 302) \mathrm{d} \tau
$$

The HW load (Q3n2) should be obtained for the hourly, daily, monthly and annual data summary.

\subsubsection{Solar Fraction of the HW Load (N300)}

The solar fraction of the HW load is calculated by:

$$
\mathrm{N} 300=\frac{1}{\mathrm{TI}} \int_{\tau_{1}}^{T_{2} 2 \mathrm{TD} 301} \frac{\mathrm{TD} 301+\mathrm{TD} 302}{\mathrm{~T}} \mathrm{~d}, \mathrm{~W} 301 \neq 0
$$

where TD301 and TD302 are the temperature differences across the HW storage tank and auxiliary heat exchangers, respectively, when there is a flow to the HW load (W301 $\neq 0$ ).

This parameter should be evaluated on a daily, monthly and annual basis.

\subsubsection{HW Electrical Energy Saved (Q311)}

The measured electrical energy used by the HW subsystem of Figure 4 is given by:

$$
\mathrm{Q} 309=\mathrm{Q} 303+\mathrm{Q} 305+\mathrm{Q} 308
$$

where $Q 303$ is the energy required to operate the circulation pump, Q305 is the energy input to the auxiliary heater $(Q 305=0$ for a fossil auxiliary), and Q308 is the HWS fraction of the ECSS operating energy. 

The calculated electrical energy required to operate a conventional hot water subsystem is given by:

$$
\mathrm{Q} 310=\mathrm{Q} 302 / \mathrm{NHWE}
$$

where Q302 is the hot water load previously described, and NHWE is the thermal efficiency of a conventional HW subsystem using electricity as an energy source.

The electrical energy saved is the difference between the calculated electrical energy to operate a conventional system having the same source and delivery temperature and usage rate as the solar energy system and the measured electrical energy required to operate the solar energy system as follows:

$$
\mathrm{Q} 311=\mathrm{Q} 310-\mathrm{Q} 309
$$

If a negative value is obtained for Q311 this implies that the particular solar HWS consumes more electrical energy than the assumed conventional system it is replacing.

\subsubsection{HW Fossil Energy Saved (Q313)}

I'he fossil energy used by the solar HWS is calculated in the following manner:

$$
\text { Q306 }=\text { HVF } \int_{\tau_{1}}^{\tau_{2}} \text { F300 d }
$$

where HVF is the fuel heating value in $B T U / L B$ and $F 300$ is the measured mass flow rate of fuel to the auxiliary in $L B / H R$.

The fossil fuel energy required to operate a conventional HWS is calculated from;

$$
\mathrm{Q} 312=\mathrm{Q} 302 / \mathrm{NHWF}
$$

where Q302 is the HW load and NHWF is the thermal efficiency of a conventional HW system using fossil fuel as an energy source.

The fossil fuel energy saved is the difference between the calculated fossil energy required to operate a conventional $\mathrm{HW}$ system and the measured fossil energy required to operate a solar energy system as follows:

$$
Q 313=Q 312-Q 306
$$



The primary performance factors are based on the SHS shown in Figure 5.

\section{6.1 Space Heating Load (Q402)}

The space heating load is the sensible energy added to the air in the building and is given by:

$$
\text { Q402 }=\int_{\tau_{1}}^{\tau_{2}} W 600 \cdot \mathrm{C} 600 \cdot \mathrm{TD} 600 \mathrm{~d} \tau
$$

where $W 600$ is the building air flow rate, C600 is the specific heat, and TD600 is the temperature rise of the air flow across all the heat exchangers in the air handling unit.

The space heating load should be determined for the hourly, monthly and seasonal data summary.

\subsubsection{Solar Fraction of Space Heating Load (N400)}

The solar fraction of the space heating load for the SHS comprised of a heat pump and electric auxiliary (SYSTEM A) is given by:

$$
\mathrm{N} 400=\int_{\tau_{1}}^{\tau_{2}}(\mathrm{Q} 406+\mathrm{Q} 407) \mathrm{d} \tau / \int_{\tau_{1}}{ }^{\tau_{2}} \mathrm{Q} 402 \mathrm{~d} \tau
$$

where Q406 is the energy delivered to the heating 1oop load heat exchanger in mode 1 , and Q407 is the energy delivered to the heat pump evaporator in modes 2 and 3.

The solar fraction of the space heating load for the SHS comprised of the fossil fuel auxiliary (SYSTEM B) is given by:

$$
\mathrm{N} 400=\int_{\tau_{1}}^{\tau_{2}} \mathrm{Q400} \mathrm{d} \tau / \int_{\tau_{1}}^{\tau_{2}}{ }_{\mathrm{Q} 402} \mathrm{~d} \tau
$$

where Q400 is the energy delivered to the heating loop load heat exchanger, and Q402 is the space heating load previously described.

This parameter should be determined for the daily, monthly and seasonal data summary .

\subsubsection{Space Heating Electrical Energy Saved (Q415)}

The electrical energy used by the heat pump system shown as system A in Figure 

5 is given by:

$\mathrm{Q} 413=\mathrm{Q} 403+\mathrm{Q} 404+\mathrm{Q} 409+\mathrm{Q} 412$

where Yứ's is the energy consumed by the heating loop circulation pump, building fan and all controls,

0404 is the energy to operate the heat pump compressor,

Q409 is the energy to the electric auxiliary heat exchanger and

Q412 is the SHS fraction of the ECSS operating energy.

The electrical energy consumed by a non-solar heat pump heating system is given by:

Q414 = Q402/NHTE

where $Q 402$ is the measured space heating load, and NHTE is a modified coefficient of performance of the non-solar heat pump system which as defined in reference [2] includes electrical energy required to operate the compressor, supply fan, return fan, outdoor alr fan and HVAC control circult.

The modifications to the equipment $\operatorname{COP}$ include the supplemental energy reauired for defrosting and auxillary heating and the reduction in performance due to cyclic operation at off design conditions. Reference [ 7] presents data describing off-design heat pump performance and the discrepancy between manufacturers performance data and measured performance primarily due to defrosting requirements.

The electrical energy saved is therefore the difference between the calculated electrical energy required to operate a conventional heating system and the measured electrical energy required to operate the solar energy system as follows:

$$
Q 415=Q 414-Q 413
$$

Space Heating Fossil Energy Saved (Q417)

The fossil energy saved by an all electric-heating system is obviously zero, therefore, to lllustrate this calculation, the fossil energy used by the alternate SYSTEM B is given by:

$$
\text { Q410 }=\text { HVF } \int_{\tau_{1}}^{\tau_{2}} \text { F400 d } \tau
$$

where HVF is the heating value of the fuel, and $F 400$ is the mass flow rate of fuel consumed by the auxiliary.

The fossil-energy consumed by a non-solar heating system must be calculated for the measured load conditions based on the performance characteristics and/or component efficiencies of the equipment as follows:

$$
\text { Q416 = Q402/NHTF }
$$



where Q402 is the space heating load and,

NHTF is the assumed thermal efficiency of the equipment.

The fuel energy saved is given by the difference between the calculated fossil energy consumption of the non-solar heating system and the measured fossil energy consumption of the solar energy system as follows:

$Q 417=Q 416-Q 410$

6.7 Space Cooling Subsystem (SCS) Primary Performance Factors

The primary performance factors of the space cooling subsystem are based on the SCS shown schematically in Figure 6.

\subsubsection{Space Cooling Load (Q502)}

The space cooling load is the total energy, including sensible and latent, removed from the air in the building and is given by:

$$
\begin{aligned}
& Q 502=\int^{\tau_{2}}\left[\mathrm{~W} 600 \cdot \mathrm{C} 600 \cdot \mathrm{TD} 600+\mathrm{W} 601 \cdot \mathrm{h}_{\mathrm{FG}}\right] \mathrm{d \tau} \\
& \text { where W600 is the building supply air flow rate, } \\
& \text { TD600 is the temperature differential across the fan-co11 unit, } \\
& \text { W601 is the flow rate of condensed water vapor from the cooling coil, } \\
& \text { and } \mathrm{h}_{\mathrm{FG}} \text { is the latent heat of vaporization of the condensate. }
\end{aligned}
$$

Alternativeiy if air side measurements are not practical, the space cooling load can be approximated from Q507 shown in Figure 6 which is the measured absorption chiller load.

\subsubsection{Solar Fraction of Space Cooling Load (N500)}

The solar fraction of the space cooling load is given by:

$$
\mathrm{N} 500=\int_{\tau_{1}}^{\tau_{2}} \mathrm{Q} 500 \mathrm{d \tau} / \mathrm{S}_{\tau_{1}}^{\tau_{2}} \mathrm{Q} 506 \mathrm{d \tau}
$$

where Q500 is the solar energy delivered to the SCS, and Q506 is the total thermal energy delivered to the absorption chiller generator consisting of solar and auxiliary thermal energy.

This parameter should be determined for the dally, monthly and seasonal data summary. 



\subsection{Space Cooling Electrical Energy Saved}

The electrical energy used by the solar assisted space cooling subsystem is given by :

$$
\mathrm{Q} 510=\mathrm{Q} 503+\mathrm{Q} 509
$$

where Q503 is the cooling subsystem operating energy including power for the heating loop circulation pump, bullding fan, absorption unit pumps, cooling tower fans and all subsystem controls, and Q509 is the space cooling subsystem fraction of the ECSS electrical energy consumption.

The electrical energy consumed by a non-solar cooling system must be calculated for the measured load conditions, source and sink temperatures using the appropriate conversion efficiency as follows:

Q511 = Q502/NCLF

where Q502 is the measured cooling load, and NCLF is the electrical efficiency of the non-solar cooling equipment.

The electrical energy saved is the difference between the calculated electric energy consumption of the conventional cooling system and the measured electric energy consumption of the solar energy system as follows:

$$
Q 512=Q 511-Q 510
$$

\subsubsection{Space Cooling Fuel Energy Saved (Q514)}

The fossil fuel energy used by the solar space cooling subsystem is given by:

$$
\text { Q508 }=\text { HVF } \int_{\tau_{1}}^{\tau_{2}} \text { F500 d } \tau
$$

where $F 500$ is the fuel mass flow rate to the auxiliary unit, and HVF is the heating value of the fuel.

The fuel energy consumed by a non-solar ccoling subsystem must be calculated for the measured load conditions based on the component performance characteristics and/or efficiency as follows: 



$$
\text { Q513 = Q502/NCLF }
$$

where Q502 is the measured space cooling load,

and NCLF is the thermal efficiency of the non-solar equipment.

The fossil fuel energy saved is given by the difference in the calculated fossil energy consumption of the non-solar cooling system and the measured fossil energy consumption of the solar energy system as follows:

$$
\text { Q514 = Q513-Q508 }
$$

\subsection{Solar Energy System/Building Sumary \\ The following primary performance factors summarize the performance of the overall solar energy system and the building.}

\subsubsection{Average Building Dry Bulb Air Temperature (N407)}

Average building temperature is an occupant utilization parameter that will have a strong influence on system performance for both heating and/or cooling and is therefore useful for comparison of alternate systems. Average dry bulb temperature should be evaluated as follows:

$$
\begin{aligned}
& \text { N400 }=\frac{1}{\mathrm{TI}} \int^{{ }^{\top} 2}{ }^{\tau_{1}} \mathrm{~T} 600 \mathrm{~d} \tau \\
& \text { where } \mathrm{T} 600 \text { is the measured building air dry bulb temperature. }
\end{aligned}
$$

This parameter should be determined for the hourly, dally, monthly and seasonal data summary.

\subsubsection{Total Auxiliary Energy (Q301, Q302, Q303, Q600)}

Auxillary energy is required for HW, space heating and space cooling whenever the solar system is not capable of meeting the demand. The amount of auxiliary energy required is determined using the same basic approach described in Section 6.4.2 for solar energy utilized and is calculated as follows:

6.8.2.1 Auxiliary Used for HW

$$
\mathrm{Q} 301=\int_{\tau_{1}}^{\tau_{2}} \mathrm{~W} 301 \cdot \mathrm{C} 302 \cdot \mathrm{TD} 302 \mathrm{d \tau}
$$



For SYSTEM A of Figure 5, the auxillary energy used for space heating is given by:

$$
\text { Q401 }=\int{ }_{\tau_{1}}^{\tau_{2}} \mathrm{W600} \cdot \mathrm{C} 602(\mathrm{TD} 600-\mathrm{TD} 601) \mathrm{d} \tau
$$

For SYSTEM B of Figure 5, the auxiliary energy used for space heating is given by :

$$
\text { Q401 }=\int_{\tau_{1}}^{\tau_{2}} \mathrm{W400} \cdot \mathrm{C} 401 \cdot \mathrm{TD} 401 \mathrm{d \tau}
$$

6.8.2.3 Auxiliary Used for Space Cooling

$$
Q 501=\int_{\tau_{1}}^{\tau_{2}} \mathrm{~W} 400 \cdot \mathrm{C} 401 \cdot \mathrm{TD} 401 \mathrm{~d} \tau
$$

6.8.2.4 Total Auxiliary Energy Utilized

The total auxiliary energy utilized is the sum of the auxiliary energy used for each application and is given by:

$$
\mathrm{Q} 600=\mathrm{Q} 301+\mathrm{Q} 401+\mathrm{Q} 501
$$

Total auxiliary energy should be determined for hourly, dally, monthly and seasonal/annual data summary.

\subsubsection{Total Operating Energy (Q601)}

The total operating energy is the thermal equivalent of the electrical energy required to run the pumps, fans, compressors and controls but excludes the electrical energy used for auxillary heating purposes. The total operating energy is composed of the individual components as follows:

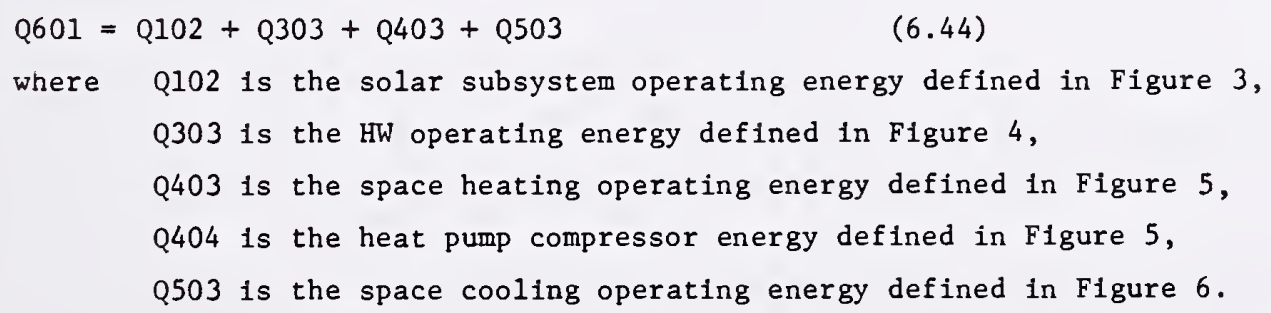



Total operating energy should be determined for the hourly, daily, monthly and seasonal/annual data summary.

\subsubsection{Total Energy Delivered to Building Load (Q602)}

The total building energy load is the sum of the IW load, the space heating load and space cooling loads previously defined, and is calculated by:

$$
\mathrm{Q} 602=\mathrm{Q} 302+\mathrm{Q} 402+\mathrm{Q} 502
$$

The total bullding energy load should be determined for the hourly, daily, monthly. seasonal/annual data summary.

\subsubsection{Total Energy Consumed (Q603)}

Total energy consumption consists of the total solar, fossil and electric energy consumed by all of the subsystems as follows:

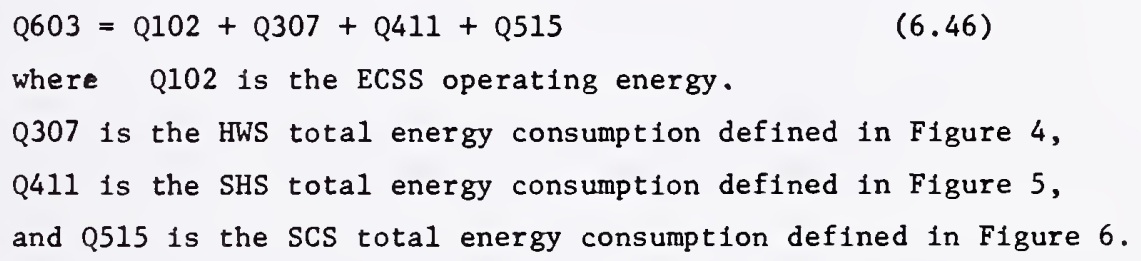

Total energy consumed should be determined for the hourly, daily, monthly and seasonal/annual data summary.

\subsubsection{Total Electric Energy Saved (Q604)}

The total electric energy saved is given by:

$$
\begin{aligned}
& \mathrm{Q} 604=\mathrm{Q} 311+\mathrm{Q} 415+\mathrm{Q} 512 \\
& \text { where Q311, Q415 and Q512 are the electric energies saved for HW, } \\
& \text { space heating and space cooling, respectively. }
\end{aligned}
$$

\subsubsection{Total Foss11 Energy Saved (Q605)}

The total fuel energy saved is given by:

$$
\mathrm{Q} 605=\mathrm{Q} 313+\mathrm{Q} 417+\mathrm{Q} 514
$$

where Q313, Q417 and Q514 are the fossil fuel energies saved for $\mathrm{HW}$, space heating and space cooling respectively.

Energy saved should be determined for the hourly, daily, monthly and seasonal/ annual data summary. 



\subsubsection{Solar Fraction of Building Load (N601)}

The solar fraction of the building load is determined by averaging the solar fractions of the individual subsystem loads as follows:

$$
\mathrm{N} 601=\frac{\int_{\tau_{1}}^{\tau_{2}}[N 300 \cdot Q 302+N 400 \cdot Q 402+N 500 \cdot 0502] d \tau}{\int_{\tau_{1}}^{{ }^{\top} 2}[Q 302+Q 402+Q 502] d \tau}
$$

where N300, N400 and N500 are the solar fractions, respectively, of the HW load, heating load and cooling load, and Q302, Q402, Q502 are,respectively, the HW load, heating load and cooling load.

\subsubsection{System Performance Factor (N602)}

The ratio of the total energy delivered to the building load to the total equivalent fossil fuel energy expended is defined to be the system performance factor and is calculated as follows:

$$
\begin{aligned}
& \mathrm{N} 602=\int^{\tau} 2 \mathrm{Q602} \mathrm{d \tau} / \mathrm{S}^{\tau}{ }^{\tau}[\mathrm{Q} 306+\mathrm{Q} 410+\mathrm{Q} 508+\underline{(0601+0305+0409)] \mathrm{d} \tau} \text { (6.50) } \\
& { }^{\tau_{1}}{ }_{1} \tau_{1}
\end{aligned}
$$

NELEC is the overall thermal efficiency for conversion of fossil energy at the power plant to electrical energy at the building.

The system performance factor should be determined for the daily, monthly and seasonal/annual data summary.

6.9 Secondary Performance Factors and Measurements

In addition to the primary system performance factors described in Section 6.3 to 6.8 , certain secondary performance evaluation factors for the ECSS and ECDS are of sufficient importance to warrant further discussion. 



\subsubsection{Daily Integrated Collector Efficiency (N100)}

The ratio of the daily integrated solar energy collected by the array to the daily integrated total solar energy incident on the array is termed the daily integrated collector efficiency and is a measure of the ability of the solar collector to convert daily incident solar radiation into thermal energy available either immediately or for storage and subsequent use thereof to support the building thermal energy load. Daily integrated collector efficlency is calculated by:

$$
\mathrm{N} 100=\int_{{ }^{\tau} 1}^{\tau_{2}} \mathrm{Q} 100 \mathrm{d \tau} / \int^{\tau_{2}}{ }^{\tau_{1}} \mathrm{Q} 001 \mathrm{~d} \tau
$$

where the numerator represents the total thermal energy collected per day for one square foot of collector and the denominator represents the total solar radiation incident on one square foot of collector per day.

\subsubsection{Collector Array Instantaneous Performance (N101, N102, N103, N104)}

The performance of a flat plate collector operating under steady state conditions can be described by the following equation:

$$
\begin{aligned}
& \frac{Q_{u}}{A_{c}}=F_{R}\left[\left(I_{T}\right)(\tau \alpha)_{e}-U_{L}\left(T_{f, I}-T_{a}\right)\right] \\
& \text { where } Q_{u}=\text { rate of useful energy extraction from the collector } \\
& A_{c}=\text { collector area, aperature } \\
& F_{R}=\text { Actual useful energy collected } \\
& \text { useful energy collected if the entire } \\
& \text { collector were at the inlet fluid temperature } \\
& I_{T}=\text { total incident solar radiation } \\
& (\tau \propto)_{e}=\text { effective transmission absorptance product of collector } \\
& \mathrm{U}_{\mathrm{L}}=\text { heat transfer loss coefficient for the collector } \\
& \mathrm{T}_{\mathrm{f}, 1}=\text { fluid temperature entering collector } \\
& \mathrm{T}_{\mathrm{a}}=\text { ambient air temperature }
\end{aligned}
$$

Defining the solar collector efficiency as the ratio of useful energy extracted from the collector to the total solar radiation incident, equation (6.52) is rearranged to give collector efficiency as follows:

$$
N 101=F_{R}\left[(\tau \alpha) e-U_{L} \frac{T_{f, 1}-T_{a}}{I_{T}}\right]
$$



A plot of equation (6.53) as a function of $\left(T_{f, 1}-T_{a}\right) / I_{T}$ for a range of operating conditions yields a first or second order curve with the $y$ axis intercept equal to $F_{R}(\tau \propto)_{e}$ and slope equal to $F_{R} U_{L}$.

If an individual collector panel is instrumented the same as the collector array with inlet and exit temperatures and flow rate monitored, or if the entire collector array is co-planer, then by evaluating the two factors $F_{R}(\tau \propto)$ and $F_{R} U_{L}$ over the life of the demonstration, degradation of the array due to changes in the $\tau^{\propto}$ product or $U_{L}$ (heat transfer loss coefficient) will be revealed.

Meaningful efficiency measurements are obtained when essentially steady state is achieved for the previous 15 minutes (three data scans, if the scan occurs every five minutes). The following measurements should be constant:

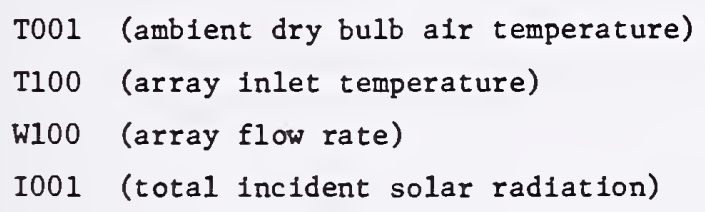

Other constraints for the procedure as obtained from reference ( 6 ) are to limit the calculation to times during which the angle between the sun and the collector array normal is less than $30^{\circ}$ degrees and the total incident solar radiation is greater than $630 \mathrm{w} / \mathrm{m}^{2}\left(200 \mathrm{BTU} / \mathrm{hrft}^{2}\right)$. If all the above constraints are satisfied, then data taken at the last data scan are used to calculate the following performance indicies.

6.9.2.1 Instantaneous Collector Array Efficiency (N101)

$$
\mathrm{N} 101=\frac{\mathrm{W} 100 \cdot \mathrm{C} 100 \cdot \mathrm{TD} 100}{\mathrm{~A}_{\mathrm{C}} \cdot \mathrm{I001}}
$$

6.9.2.2 Collector Panel Efficiency Factor (N102)

-

$$
\mathrm{N} 102=(\mathrm{T} 100-\mathrm{T} 001) / \mathrm{I} 001
$$

\section{9.2.3 Collector Panel Factors (N103, N104)}

The newly calculated values of the parameters $\mathrm{N} 101$ and $\mathrm{N} 102$ are used together 

with previously calculated values of $\mathrm{N} 101$ and $\mathrm{N} 102$ using the previously described slope/intercept linear curve fit and new values of the collector panel factors N103 and N104 are obtained, where:

$$
\begin{aligned}
& \mathrm{N} 103=F_{R}(\tau \alpha) \mathrm{e} \\
& \text { and, } \\
& \mathrm{N} 104=F_{R} U_{L}
\end{aligned}
$$

The above described procedure should be performed at the start of each hour until a data scan occurs which satisfies all specified constraints. For that scan, the calculated values of $\mathrm{N} 101, \mathrm{~N} 102, \mathrm{~N} 103$ and $\mathrm{N} 104$ are reported in the hourly data summary and no further scans are required for the remainder of the hour. At the start of the next hour the procedure is repeated.

\subsubsection{Collector Heat Exchanger Effectiveness (N105)}

For the ECSS where the thermal capacitance of the storage container is much greater than the flow capacitance of the collector loop, the collector heat exchanger effectiveness is given by:

$$
\mathrm{N} 105=\frac{1}{\mathrm{TI}} \int_{\tau_{1}}^{\tau_{2}}(\mathrm{TD} 101) /(\mathrm{T} 101-\mathrm{T} 200) \mathrm{d} \tau
$$

Evaluation of collector heat exchanger effectiveness can be done on a monthly and annual basis.

6.9.4 HW Heat Exchanger Effectiveness (N106)

The hot water heat exchanger effectiveness is determined as follows:

$$
\mathrm{N} 300=\frac{1}{\mathrm{TI}} \int_{\tau_{1}}^{\tau_{2}}(\mathrm{Tn} 300) /(\mathrm{T} 200-\mathrm{T} 300) \mathrm{d} \tau
$$

Die to the requirement for double walled heat exchangers to prevent any possible contamination of potable water with non-potable water, the HW heat exchangers may present a substantial thermal resistance to heat transfer and thereby reduce the amount of solar energy available. Any tendency for fouling of the heat exchangers would be revealed by changes in the calculated values of heat exchanger effectiveness. 



\subsubsection{Storage Subsystem Efficiency (N108, N109)}

The storage subsystem efficiency is defined as the ratio of the sum of the output of stored thermal energy and the increase in stored thermal energy to the Input of thermal energy to storage as follows:

$$
\mathrm{N} 108=\frac{\delta_{\tau_{1}}^{{ }^{\tau}} 2}{\int^{{ }^{\tau} 2}(0201+0202) d \tau}
$$

where Q201 is the hourly output of thermal energy from the storage device,

Q202 is the hourly increase in stored energy, and $Q 200$ is the hourly input of thermal energy to the storage device.

The normal source of thermal energy input to storage is from the solar energy collectors, however, other thermal energy sources, such as off-peak auxillary energy must also be included. Where more than one storage contalner is used, individual evaluation is required.

Storage efficiency is related to the subsystem heat losses which may be elther deliberate (such as the dumping of energy to amblent) or non-deliberate due to a1r leakage or insulation losses. Heat loss through insulation is related to the following parameter:

$$
\mathrm{N} 109=\frac{1}{\mathrm{TI}} \int_{\tau_{1}}^{\tau_{2}}(\mathrm{~T} 200-\mathrm{T} 201) \mathrm{d} \tau
$$

where T200 is the average storage medium temperature, and T201 is the ambient temperature surrounding the storage container.

Evaluation of these parameters (N108 and N109) should be performed for the monthly and seasonal/annual data summary.

\subsubsection{Average HW Load Temperature (N307)}

The average HW delivery temperature to the load is a significant parameter in the comparison of alternate HW solar systems, in that this temperature is controlled by the occupants and subsystem performance w1ll depend on the actual control point setting.

$$
\mathrm{N} 307=\frac{1}{\mathrm{TI}} \int_{\tau_{1}}^{\tau_{2}}(\mathrm{~T} 301+\mathrm{TD} 301+\mathrm{TD} 302) \mathrm{d} \tau
$$



where T301 is the make-up water temperature,

TD301 is the temperature difference across the hot water storage, and TD302 is the temperature difference across the HW auxiliary heat exchanger.

\subsubsection{Average Gallons HW Consumed (N308)}

The time of day at which hot water is used and the total dally consumption are significant parameters in evaluating performance and comparison of alternate HW solar systems. The average HW consumption in gallons is given by :

$$
\mathrm{N} 308=.12 \int_{\tau_{1}}^{\tau_{2}} \mathrm{~W} 301 \mathrm{~d} \tau
$$

where W301 is the measured flow rate in $\mathrm{lbs} / \mathrm{hr}$.

The HW consumption should be determined for the hourly, dafly, monthly and annual data summary.

\subsubsection{Energy Conversion Equipment Coefficient of Performance}

Enegry conversion equipment uses electrical or thermal energy to ralse heat from 2 low temperature source to a higher temperature sink and is rated by the ratio of the useful heat transferred to the energy input. Useful heat transferred consists of heat delivered to the sink in the heating mode and heat extracted from the source in the cooling mode.

\subsubsection{Space Heating Equipment COP (N404)}

Measurement of the Coefficlent of Performance (COP) is desired for energy conversion equipment such as heat pumps in which the useful thermal energy delivered to the bullding is significantly greater than the electrical energy consumed. Referring to the heat pump system shown as SYSTEM A in Figure 5, Space Heating $\operatorname{COP}$ is calculated from:

$$
\mathrm{N} 404=\int_{\tau_{1}}^{\tau_{2}}{ }_{\mathrm{Q} 407} \mathrm{d \tau} / \int_{\tau_{1}{ }^{\tau_{2}}}(\mathrm{Q} 403+\mathrm{Q} 404) \mathrm{d} \tau
$$

where Q407 is the useful thermal energy added to the building supply air from the heat pump,

Q403 is the electrical energy required to operate the SHS pump, fans and controls, 

Q404 is the electrical energy required to operate the heat pump compressor.

Th1s parameter should be determined for the dally, monthly and seasonal data summary.

6.9.8.2 Space Cooling Equipment COP (N503)

Measurements of the Coefflclent of Performance (COP) are desired for thermally actuated equipment such as the absorption chiller for Figure 6 and for other equipment such as solar assisted Rankine Cycle equipment or dessicant dehumidification systems as appropriate.

Referring to the absorption chiller shown in Figure 6, the Coefficient of Performance is calculated as:

$$
\mathrm{N} 503=\int_{\tau_{1}}^{\tau_{2}} \mathrm{Q507/S_{ \tau _{1 } } ^ { \tau _ { 2 } }} \mathrm{Q506} \mathrm{d} \tau
$$

where Q507 is the useful thermal output of the absorption chiller as defined in Figure 6, and Q506 is the thermal energy input to the absorption chiller as defined in Figure 6. 

The uncertainty of determining the performance evaluation factors ior a particular solar energy system/building/climate combination is related to the data requirement accuracy for sensor signal condftioning, data acquisition sampling rate and data processing method. Non-instrumentation variables such as building heating or cooiing load, occupancy, conventional heating and air conditioning equipment operation and meteorological parameters must also be considered when comparing the performance of solar energy systems in different bulldings or climatic regions.

To establish acceptable tolerances in the performance evaluation factors, the contribution of sensor accuracy and sampling rate error to the total performance factor uncertainty must be derived and set in perspective by comparison with the pertinent non-instrumentation sources of the performance factor uncertainty. The approach, rationale and sensitivity analysis for several performance factors are described in the following sections:

- Sensitivity Analysis Methods

- Specific Error Analysis

- Overall Instumentation Errors

- Recommended Sampling Rates and Data Requirement Accuracies

7.1 Sensitivity Analysis Methods

Two methods are in general use for combining precision errors in measuring several variables to estimate the error in a calculated function of those variables. The particular method used depends on whether the component errors are considered to be absolute limits or statistical bounds (such as $3 \sigma$ limits) the form of the relationship between the variables and the calculated function, and the specification of component errors (percent of reading or absolute in terms of neasured units).

\subsubsection{Absolute Limits}

If the component precision errors are considered to be absolute limits and a functional relationship exists such that

$$
T=f(x, y, z)
$$

If the component errors in $x, y$, and $z$ are $\Delta x, \Delta y$ and $\Delta z$ respectively, and $f(x, y, z)$ is expanded in a Taylor series; then: 


$$
\begin{aligned}
& f(x+\Delta x, y+\Delta y, z+\Delta z)=f(x, y, z)+\frac{\partial f \Delta x}{\partial x}+\frac{\partial f}{\partial y} \Delta y+\frac{\partial f}{\partial z} \Delta z \\
& +1 / 2\left[\frac{\partial^{2} f \Delta x^{2}}{\partial x^{2}}+\frac{\partial^{2} f}{\partial y^{2}} \Delta y^{2}+\frac{\partial^{2} f}{\partial z^{2}} \Delta z^{2}\right]+\ldots(7.2)
\end{aligned}
$$

Neglecting the second order and larger terms because $\Delta x, \Delta y, \Delta z$ are assumed small $(\sim 1 \%)$, the total measurement error is:

$$
\Delta f=\left|\frac{\partial f}{\partial x} \Delta x\right|+\left|\frac{\partial f}{\partial y} \Delta y\right|+\left|\frac{\partial f}{\partial z} \Delta z\right|
$$

In the general case for a function $R$ (a function of any number of variables) the error can be expressed as:

$$
\Delta R=\sum_{i=1}^{n},\left|\frac{\partial R}{\partial x_{1}} \Delta x_{1}\right|
$$

\subsubsection{Statistical Bounds}

In the absence of a function relating the component error sources, a general equation can be used to find the measurement error. If the measurement is defined by $R\left(x_{1}, x_{2} \ldots . x_{n}\right)$, where $R$ is the desired measurement and $\Delta x_{1}, \ldots \Delta x_{n}$ are the individual error sources, the following equation can be used:

$$
\Delta R=\left[\sum_{i=1}^{n}\left(\frac{\partial R}{\partial x_{i}} \Delta x_{i}\right)^{2}\right]^{1 / 2}
$$

This equation assumes that the errors are random and uncorrelated. The precision errors will be expressed as $\pm \Delta \mathrm{X}$. As a probable error in either of the two cases (functional or non-functional relationship), the true value of $R$ will be between $R-\Delta R$ and $R+\Delta R$ for some percentage of all readings. The percentage involved depends upon the error expressions used for the component errors.

\subsubsection{Error Analysis - Daily Integrated Collector Array Efficiency}

An error analysis of the calculations of dally integrated collector array efficlency is presented to illustrate using both methods discussed in the preceding paragraphs. 

From Figure 3, the daily integrated collector array efficiency is calculated as:

$$
\mathrm{N} 100=\int Q 100 \mathrm{~d} \tau / \int Q 001 \mathrm{~d} \tau=\int \mathrm{T} 100 \cdot \mathrm{C} 100 \cdot \mathrm{TD} 100 \mathrm{~d} \tau / \mathrm{Ac} \int \mathrm{I001 \textrm {d } \tau}
$$

If the component errors are indepencent of time than the uncertainty in the integrated daily efficiency is the same as the uncertainty in the instantaneous efficiency given by performance factor N101 as:

$$
\mathrm{N} 101=\frac{\mathrm{W} 100 \cdot \mathrm{C} 100 \cdot \mathrm{TD} 100}{\mathrm{Ac} \cdot \mathrm{I001}}
$$

For purposes of illustration, the following reference conditions and tolerances in each variable are assumed:

$$
\begin{aligned}
& \mathrm{A}_{\mathrm{C}}=32 \mathrm{ft}^{2} \pm 1 \% \text { (assumption) }\left( \pm .32 \mathrm{ft}^{2}\right) \text {, collector area } \\
& \mathrm{W} 100=1 \mathrm{GAL} / \mathrm{HR} \mathrm{fr}^{2} \times 32 \mathrm{ft}^{2}=269.91 \mathrm{lb} / \mathrm{hr} . \pm 2 \% \mathrm{FS}( \pm 5.4 \mathrm{lb} / \mathrm{hr}), \text { collector flor } \\
& \mathrm{C} 100=1.0 \mathrm{BTU} / 1 \mathrm{~b}{ }^{\circ} \mathrm{F} \text {, spectfic heat (NO TOLERANCE) } \\
& \mathrm{TD} 100=18 \pm 1.7 \%\left( \pm 0.3^{\circ} \mathrm{F}\right) \text {, temperature rise through collector } \\
& \text { I001 }=300 \mathrm{BTU} / \mathrm{hr} \mathrm{ft}^{2} \pm 3 \%\left( \pm 9 \mathrm{BTU} / \mathrm{hr} \mathrm{ft}^{2}\right. \text { ), incident solar radiation }
\end{aligned}
$$

The nominal array efficiency is determined from equation (7.7) by substitution of the assumed variables at the reference point as:

$$
\mathrm{N} 101=\frac{(270) \frac{\mathrm{LB}}{\mathrm{HR}}(1.0) \frac{\mathrm{BTU}}{\mathrm{LB}}{ }^{\circ} \mathrm{F}(18.0){ }^{\circ} \mathrm{F}}{(32) \mathrm{ft}^{2} \quad(300) \frac{\mathrm{BTU}}{\mathrm{hr}} \mathrm{ft}^{2}}=.506
$$

Equation 7.7 is differentiated with respect to each variable having a non-zero tolerance and each partial derivative is evaluated at the reference condition as follows:

$$
\begin{aligned}
& \frac{\partial N 101}{\partial W 101}=\frac{C 101 \cdot T D 100}{A_{C} \cdot I 001}=\frac{(1.0)(18 .)}{(32 .)(300 .)}=.0019 \\
& \frac{\partial N 101}{\partial T D 100}=\frac{W 100 \cdot C 100}{A_{C} \cdot I 001}=\frac{(270 .)(1.0)}{(32 .)(300 .)}=.0281
\end{aligned}
$$





$$
\begin{aligned}
& \frac{\partial N 101}{\partial A_{C}}=-\frac{W 100 \cdot C 100 \cdot T D 100}{A_{C}{ }^{2} \cdot I 001}=-\frac{(270)(1.0)(18.0)}{(32)^{2}(300 .)}=-0.0158 \\
& \frac{\partial N 101}{\partial I 001}=-\frac{W 100 \cdot C 100 \cdot T D 100}{A_{C} \cdot(I 001)^{2}}=-\frac{(270)(1.0)(18.0)}{(32)(300)^{2}}=-.0017
\end{aligned}
$$

The error by absolute limits is obtained by substitution of the partial derivatives and tolerances in equation $(7-4)$ as follows:

$$
\begin{aligned}
\Delta \mathrm{N} 101 & =|(.0019)(5.4\}+|(.0281)(.03)+|(-0.0158)(.32)|+\mid-.0017)(9.0) \mid \\
\Delta \mathrm{N} 101 & =.0390 \\
\mathrm{~N} 101 & =.506 \pm .039
\end{aligned}
$$

Thus the calculated value of instantaneous efficiency based on absolute error limits indicates an uncertainty of \pm 7.7 percent.

The error by statistical bounds is obtained by substitution of the partial derivatives and tolerances in equation (7.5) as follows:

$$
\begin{aligned}
\mathrm{N} 101 & =\left[(.0019 \times 5.4)^{2}+(.0281 \times 0.3)^{2}+(-0.0158 \times .32)^{2}+(-.0017 \times 9.0)^{2}\right]^{1 / 2} \\
\Delta \mathrm{N} 101 & =.021 \\
\mathrm{~N} 101 & =.506 \pm .021
\end{aligned}
$$

The statistical or rms method indicates about a \pm 4.1 percent uncertainty in the calculation of collector efficiency. As indicated by the sample calculations the absolute limit method gives a larger error value than the statistical method. Because of the randomness of the measurement process for the solar energy systems (i.e., errors among the many different sensors are not correlated), the statistical method is used for the uncertainty and error analysis calculations reported in this document.

Derivation of the Overall Instrumentation Accuracies

Instrumentation accuracies are caused by sampling errors and by systematic sensor errors due to inaccurate calibration, drift, and non-linearities. To evaluate the effect of sensor and sampling errors on the performance factors, the following information is necessary: the analytical expression for each 

performance factor, the range of loads and operating points for the solar energy systems and the sampling period. Application of the error analysis method to determine the uncertainty in solar energy system conversion efficiency is described to illustrate the analysis performed for most of the primary factors.

\subsubsection{Temperature Sensor Accuracy}

There are several ways of expressing temperature sensor accuracy. If all temperature sensors used to compute energy flows are differential sensors, meaning that a temperature rise or drop, not an absolute temperature is measured then accuracy of measuring a temperature differential is expressed as percent (degrees accuracy in measuring the differential divided by the total differential). If the differential is to be measured using two sensors, then the accuracy is the square root of the sum of the individual accuracies squared divided by the temperature difference or.

$$
\text { accuracy }=\sqrt{\sigma_{1}^{2}+\sigma_{2}^{2}} / \Delta T
$$

Thus, a $2 \%$ load temperature accuracy requirement and $20^{\circ} \mathrm{F}$ assumed load temperature difference means that the temperature accuracy should be $.4^{\circ} \mathrm{F}$ for a differential sensor and $.3^{\circ} \mathrm{F}$ for individual sensors. Similarly, a $4 \%$ accuracy and $10^{\circ} \mathrm{F}$ rise across a collector requires a temperature accuracy of $.4^{\circ} \mathrm{F}$ differential or $.3^{\circ} \mathrm{F}$ for individual sensors.

Other combinations of flow and temperature sensors which fulfill instrumentation accuracy requirements can be determined from Figures 9 and 10 . These two graphs were computed from the two error equations that constrain flow and temperature sensor errors.

\subsubsection{A Description of Sampling Errors}

Sampling errors develop when a signal waveform is not sampled quickly enough so that the exact waveform can be reproduced. Because many solar parameters change quite rapidly (e.g., auxiliary fuel flow or insolation), it is not Easible to sample to reproduce the entire waveform. However, it is possible by sampling a large number of times to statistically reproduce the mean of a waveform. For instance, for a monthly measurement period and a 10-minute sample interval, each parameter mean is based on approximately 2,000 samples. It is the statistics of large numbers which governs sample accuracy (provided correlation effects do not arise). 



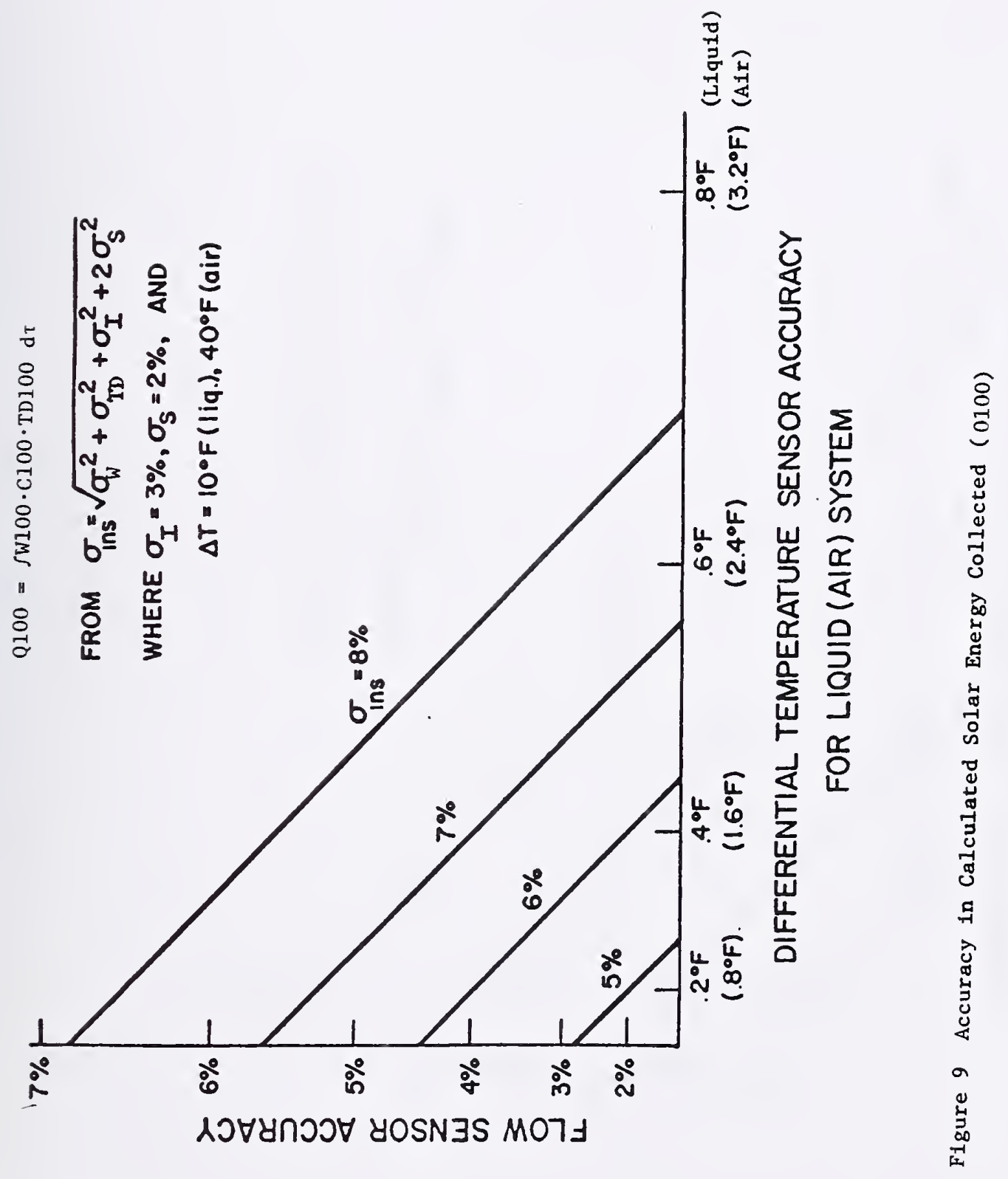





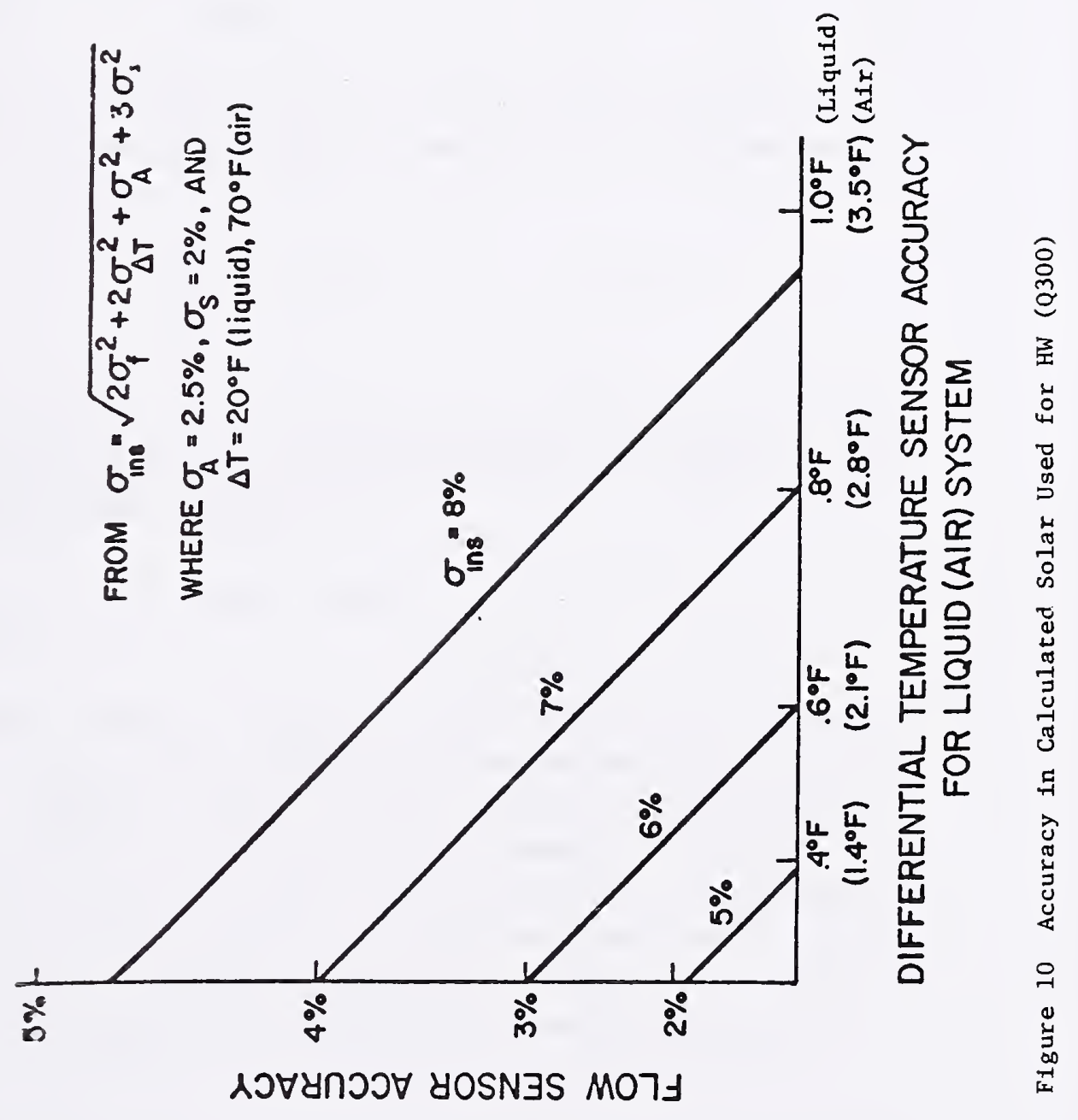



In general, lengthening the sampling period (e.g., froz one month to one year) by some factor $k$ decreases sampling error by $1 / \sqrt{k}$. S1mllarly, shortening the sampling interval (e.g., from 30 minutes to 5 minutes) decreases sampling error.

The errors due to sampling can be predicted for the three comon types of signals. "ON-OFF," "slowly varying," and "rapidly varying." Error in measuring "ON-OFF" signals is a function of the total number of samples 5 and the duty cycle, $d$

$$
\sigma=\sqrt{\frac{1-d}{N d}} \quad(O N-O F F)
$$

For "slowly varying" signals (signals that cycle much ferer than once in $2 \pi$ samples) error is a function of the number of samples, 5 , and the mean percent change of the signal during one sample interval, a:

$$
\sigma<\frac{a}{\sqrt{N(1-a)}} \quad \text { (slowly varying) }
$$

For "rapidly varying" signals (signals that cycle much faster than once every $2 \pi$ samples), error is a function of the number of samples, $N$, and the ratio of the varying to steady signal component, $b$ :

$$
\sigma<\frac{b}{\sqrt{N}(1-b)} \quad \text { (rapidly varying) }
$$

Decreasing the sample interval by a factor of $k$ results in an error decrease of approximately $1 / k$ for "ON-OFF", $1 / k \sqrt{k}$ for "slowly varying" and $1 / \sqrt{k}$ for "rapidly varying." Examples of "ON-OFF", slowly varyine, and rapidly varying signals might be auxiliary input to load, storage temperature, and insolation, respectively, for a 2 minute to 60 minute sampling interral. Actual standard deviations for different sampling rates and periods have jeen computed for insolation, collector output, auxiliary output, etc., $4 \equiv=\Sigma \bar{\xi}$ Colorado State House No. 1 data [5]. Typical data for insolation values integrated from measurements made at different intervals are listed in ZE氵ies $2 \mathrm{a}$ and $2 \mathrm{~b}$.

Assuming the 2 minute sampling rate represents the mos= Ec=urate value obtained, the actual sampling error results are listed in Table $3=-$ scaled to different sampling intervals and periods. There is wide variatic $=t=$ the monitoring interval required by the different parameters. For example, the ciysis indicates insolation need only be monitored once every 15 minutes $=2$ maintain a weekly variance below $1 \%$, but auxiliary sampling with a non-inz三srating sensor must be made. 

Table $2 a$ CSU Sampled Data Using Different Sampling Intervals (Sumer 1975)

\section{Parameter Insolation [5]}

\begin{tabular}{lcccccc} 
Date & \multicolumn{7}{c}{ Minutes } & & & \\
& 2 & 5 & 10 & 20 & 30 & 60 \\
\hline $6 / 21$ & 1132.9 & 1130.0 & 1118.9 & 1128.3 & 1124.2 & 1160.7 \\
$7 / 9$ & 1111.3 & 1119.0 & 1089.9 & 1100.4 & 1035.5 & 995.4 \\
$7 / 13$ & 1768.2 & 1776.2 & 1772.6 & 1763.9 & 1759.3 & 1765.7 \\
$7 / 17$ & 1255.3 & 1266.6 & 1270.9 & 1273.0 & 1242.9 & 1227.0 \\
$7 / 21$ & 1248.0 & 1271.6 & 1254.3 & 1241.1 & 1153.9 & 1072.2 \\
$7 / 23$ & 1263.4 & 1246.8 & 1253.1 & 1204.3 & 1259.7 & 1271.7 \\
$7 / 25$ & 1815.2 & 1811.4 & 1809.0 & 1799.1 & 1792.0 & 1767.3 \\
$7 / 27$ & 1850.4 & 1846.6 & 1840.5 & 1827.7 & 1815.1 & 1779.6 \\
$7 / 29$ & 1564.5 & 1558.1 & 1534.4 & 1515.9 & 1633.1 & 1513.0 \\
$7 / 31$ & 1205.6 & 1207.8 & 1245.6 & 1229.3 & 1219.8 & 1233.3 \\
S.D per day & & $0.8 \%$ & $1.3 \%$ & $2.0 \%$ & $3.3 \%$ & $5.3 \%$
\end{tabular}

Table 2b Parameter Insolation (Winter 1974-75)

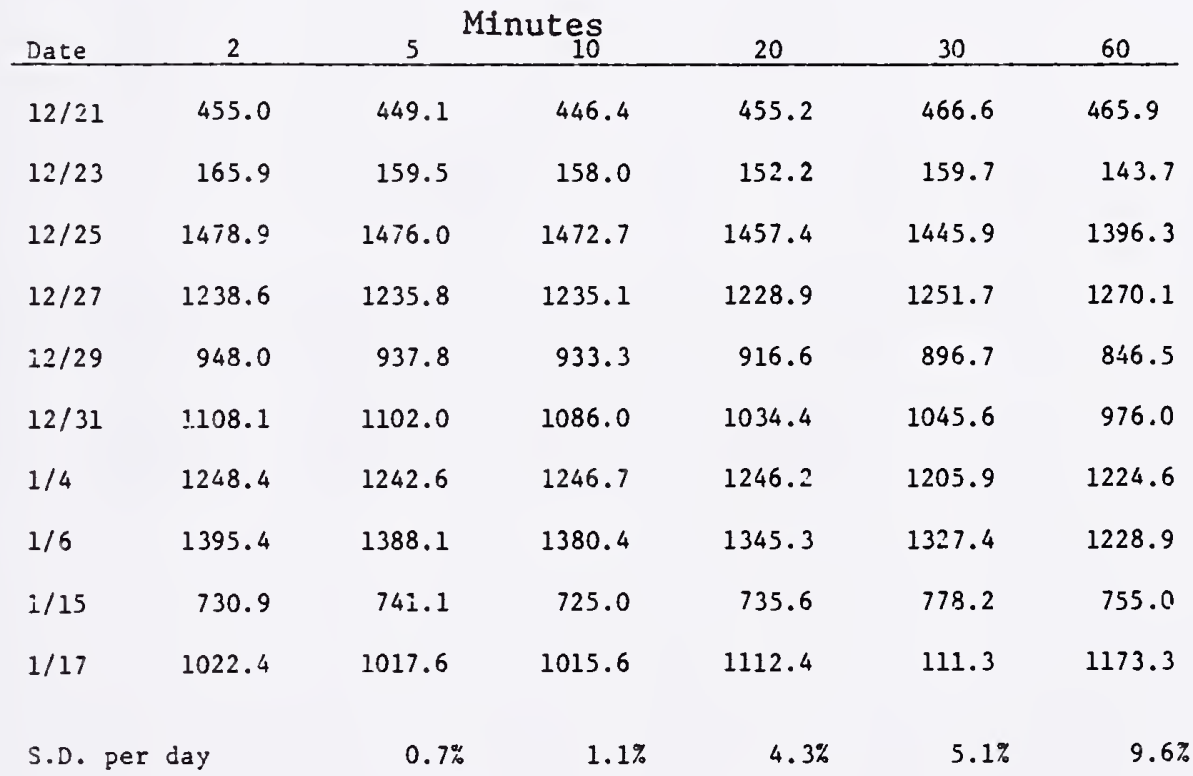



TABLE 3

RANGE OF MEASURED DAILY VARIANCE WITH DATA EXTRAPOLATED FOR WEEKLY, MONTHLY, AND YEARLY PERIODS.

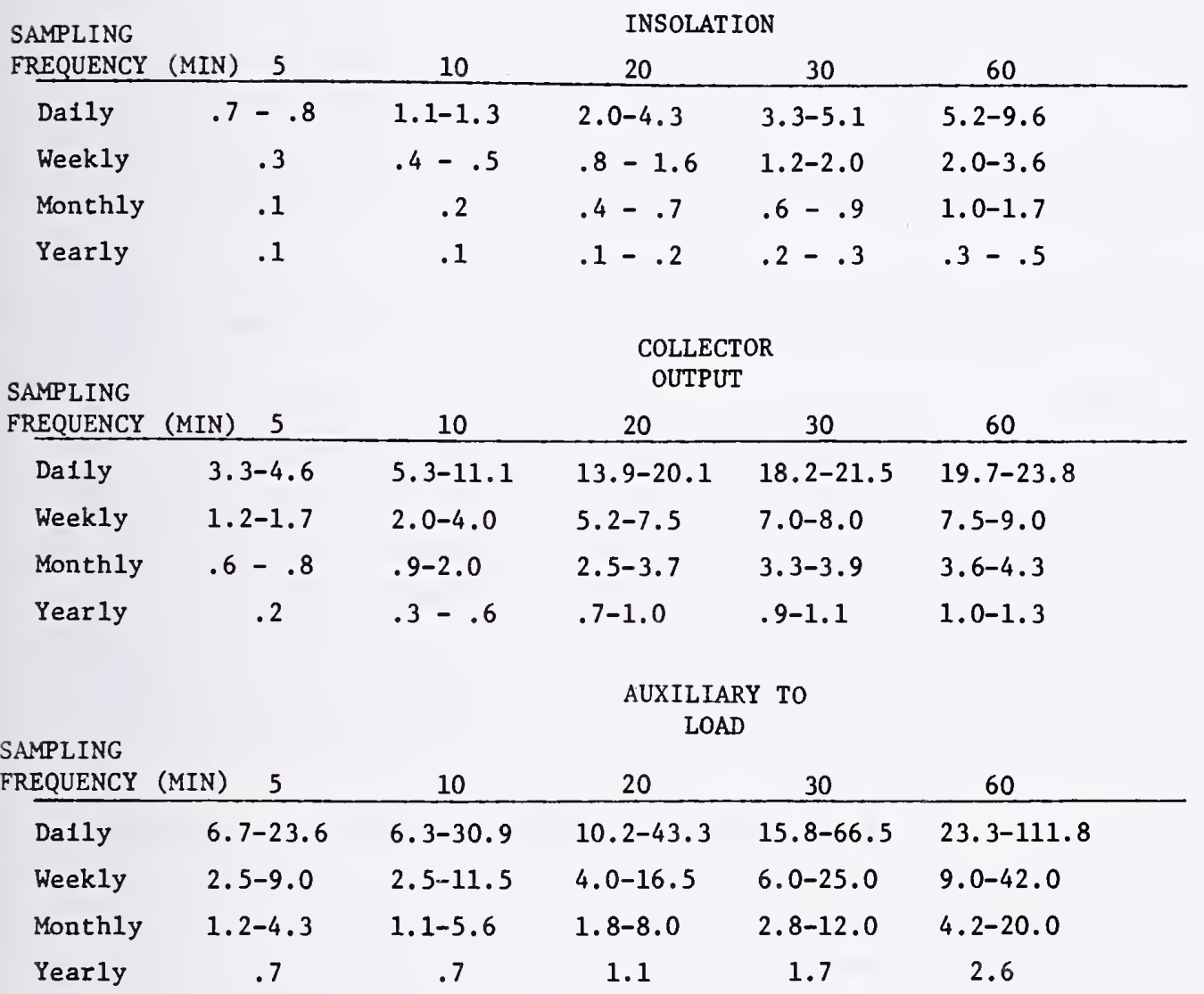



Using the CSU data and the suggested sampling accuracies from Section 7.2.2, sampling rates for various sensors are determined as listed in Table 5 .

\begin{tabular}{|l|c|c|c|}
\hline Data Requirements & Weekly Summations & Monthly Summations & Yearly Summat1on \\
\hline & (minutes) & (minutes) & (minutes) \\
load flow & $<5$ & 5 & 30 \\
collector flow and & 5 & 10 & 60 \\
temperature & $<5$ & 5 & 60 \\
auxiliary input & 30 & 60 & 60 \\
insolation & & & $1974-74$.
\end{tabular}

Based on insolation at Colorado State University, Fort Collins, Col. 1974-74.

Table 4 Sampling Rates as a Function of Time Interval for Several Data Requirements Types

Sensors requiring sampling rates greater than once every five minutes can be continuously integrated and then sampled. It is recognized that the data ut1lized is for a specific system, load and climatic conditions and will only provide estimates of sample rates for other combinations of these factors. For example, insolation sample rates are a function of the weather variability and a rate of 3 minutes is used in the Washington, D.C. area to obtain an uncertainty of about $1 \%$ [8]. A sampling rate of 5 minutes or less is recomended for both insolation and auxiliary energy related measurements.

\subsubsection{Solar Energy Conversion Efficiency (N111)}

The conversion efficiency of a heating and HW solar energy system is determined by dividing the solar energy delivered to the load by the available insolation.

Instrumentation uncertainty ( $\sigma_{\text {INS }}$ ) is given by:

$$
\begin{aligned}
& \sigma_{\mathrm{INS}}^{2}=\left(\sigma_{\mathrm{W}_{1}}^{2}+\sigma_{\mathrm{TD}}^{2}+\sigma_{\mathrm{S}_{1}}^{2}\right)\left(\frac{\mathrm{Q} 300}{\mathrm{Q} 300+\mathrm{Q} 400}\right)^{2}+\left(\sigma_{\mathrm{W}_{2}}^{2}+\sigma_{\mathrm{TD}_{2}}^{2}+\sigma_{\mathrm{S}_{2}}^{2}\right)\left(\frac{\mathrm{Q} 400}{\mathrm{Q} 300+Q 400}\right)^{2} \\
& +\sigma_{\mathrm{I}}^{2}+\sigma_{\mathrm{S} 001}^{2}
\end{aligned}
$$

where $\sigma_{\mathrm{w}}$ and $\sigma_{\mathrm{TD}}$ are uncertainties in solar-to-load flow and temperature difference sensors, $\sigma_{S}$ is the sampling uncertainty, Q300 and Q400 is solar energy to hot water and heating respectively, $\sigma_{I}$ is insolation sensor uncertainty, and $\sigma_{\text {SO01 }}$ is the insolation sampling uncertainty. 

Assuming conditions such that Q300 $>Q 400$, the instrumentation accuracy 1s:

$$
\sigma_{\mathrm{INS}}^{2}=\sigma_{\mathrm{W}_{1}}^{2}+\sigma_{\mathrm{TD}_{1}}^{2}+\sigma_{\mathrm{S}_{1}}^{2}+\sigma_{\mathrm{I}}^{2}+\sigma_{\mathrm{S} 001}^{2}
$$

The sensor accuracy and sampling rate accuracy necessary to achieve a $6 \%$ instrumentation uncertainty is determined as described in the following sections.

\subsubsection{Sensor Accuracy}

The two worst case RMS instrumentation error equations that determine necessary sensor accuracies for system and collector array efficlencies respectively are

$$
\begin{aligned}
& \sigma_{\text {INS }}^{2}=\sigma_{\mathrm{W} 301}^{2}+\sigma_{\mathrm{TD} 301}^{2}+\sigma_{\mathrm{IO01}}^{2}+\sigma_{\mathrm{S} 300}^{2}+\sigma_{\mathrm{S} 001}^{2} \\
& \sigma_{\mathrm{INS}}^{2}=\sigma_{\mathrm{W} 100}^{2}+\sigma_{\mathrm{TD} 100}^{2}+\sigma_{\mathrm{I} 001}^{2}+\sigma_{\mathrm{S} 100}^{2}+\sigma_{\mathrm{S} 001}^{2}
\end{aligned}
$$

where

$$
\begin{aligned}
& \sigma_{\mathrm{W} 301}=\sigma_{\mathrm{W} 100}=\text { flow sensor accuracles } \\
& \sigma_{\mathrm{TD} 301}=\text { load temperature sensor accuracy }\left(20^{\circ} \mathrm{F} \text { differential }\right) \\
& \sigma_{\mathrm{TD} 100}=\text { array temperature sensor accuracy }\left(10^{\circ} \mathrm{F} \text { differential }\right) \\
& \sigma_{\mathrm{I} 001}=\text { Insolation sensor accuracy } \\
& \sigma_{\mathrm{S} 300}=\text { sampling error for solar or auxiliary to load } \\
& \sigma_{\mathrm{S} 001}=\text { sampling error for insolation } \\
& \sigma_{\mathrm{S} 100}=\text { sampling error for collector output }
\end{aligned}
$$

On the basis of performance a reasonable strategy for initial selection of sensor accuracy and sampling rate is to balance the error terms of the error equations. For example, using the instrument error equation for system conversion efficiency and a $6 \%$ instrument error criterion, the error terms would be balanced according to:

$$
\frac{6 \%}{\sqrt{5}} \simeq 2.7 \% \simeq \sigma_{\mathrm{W} 301} \simeq \sigma_{\mathrm{TD} 301} \simeq \sigma_{\mathrm{I} 001} \simeq \sigma_{\mathrm{S} 300} \simeq \sigma_{\mathrm{S} 001}
$$



Using this term balancing strategy, sensor and sampling accuracies are selected and theratively refined to reasonable values using tradeoff charts shown in Figures 9 and 10 . The results for the projected sensor accuracy and sampling requirements are as follows:

\begin{tabular}{|l|c|c|}
\hline Sensor & Sensor Accuracy & Sampling Accuracy \\
\hline & $+2 \%$ & $\pm 2 \%$ \\
load flow & $\pm 2 \%$ & - \\
load temp. & $\pm 2 \%(1 i q) \pm 3 \%($ air $)$ & $\pm 2 \%$ \\
collector flow & $\pm 4 \%(11 q) \pm 3 \%($ air $)$ & - \\
collector temp. & $\pm 3 \%$ & $\pm 2 \%$ \\
insolation & $\pm 2.5 \%$ & $\pm 2 \%$ \\
auxiliary input & & \\
\hline
\end{tabular}

\subsection{SUMMARY}

The final instrumentation accuracy and performance factor uncertainty will depend upon economic tracieoifs of sensor cost, installation and maintenance.

Using the instrument error equations and selecting a desired instrumentation uncertainty of $6 \%$ as being reasonable and achievable, the values of sensor accuracy and sampling rate were assigned to the various measurements as shown in Table 5 . 



\begin{tabular}{|c|c|c|c|c|c|}
\hline TYPE & DESIGN & DATA REOUIREMENT & RANGE & ACCURACY & MRASUREMENT FREOUENCY \\
\hline 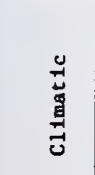 & $\begin{array}{l}1001 \\
1002 \\
\text { TOO1 } \\
\text { VOO1 } \\
\text { DOO1 } \\
\text { TOO2 }\end{array}$ & $\begin{array}{l}\text { Total Radiation } \\
\text { Diffuse Radiation } \\
\text { Outdoor DB Temperature } \\
\text { Wind Velocity } \\
\text { Wind Direction } \\
\text { Outdoor Wet Bulb Temp }\end{array}$ & $\begin{array}{l}0-350 \\
0-100 \\
-20 \text { to } 120 \\
0 \text { to } 100 \\
0 \text { to } 360 \\
32 \text { to } 90\end{array}$ & $\begin{array}{l} \pm 3 \% \mathrm{Btu} / \mathrm{ft}^{2}-\mathrm{hr} \mathrm{FS} \\
\pm 6 \% \mathrm{Btu} / \mathrm{ft}^{2}-\mathrm{hr} \text { FS } \\
\pm 2{ }^{\circ} \mathrm{F} \\
\pm 3 \mathrm{mph} \\
\pm 20^{\circ} \mathrm{F} \\
\pm 2{ }^{\circ} \mathrm{F}\end{array}$ & $\begin{array}{l}5 \mathrm{~min} \text { or less } \\
5 \mathrm{~min} \\
\mathrm{Avg} / \mathrm{hr} \\
\text { Avg } / \mathrm{hr} \\
\text { Avg } / \mathrm{hr} \\
\mathrm{Avg} / \mathrm{hr}\end{array}$ \\
\hline 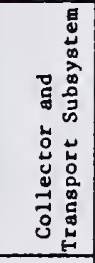 & $\begin{array}{r}\text { T100 } \\
\text { TD100 } \\
\text { T101 } \\
\text { TD101 } \\
\text { T102 } \\
\text { W100 } \\
\text { PD100 }\end{array}$ & $\begin{array}{l}\text { Collector Inlet Temp } \\
\text { Collector Temp Diff } \\
\text { Storage Inlet Temp } \\
\text { Storage Temp Diff } \\
\text { Collector Absorber Temp } \\
\text { Collector Flow Rate } \\
\text { Collector Press Diff }\end{array}$ & $\begin{array}{l}40 \text { to } 220 \\
-10 \text { to }+50 \\
60 \text { to } 230 \\
0 \text { to } 100 \\
30 \text { to } 450 \\
\text { varies } \\
\text { varies }\end{array}$ & $\begin{array}{l} \pm 0.5^{\circ} \mathrm{F} \text { (L) } \pm 1^{\circ} \mathrm{F}(\mathrm{A}) \\
\pm 0.3^{\circ} \mathrm{F} \text { (L) } \pm 1.2^{\circ} \mathrm{F}(\mathrm{A}) \\
\pm 0.5^{\circ} \mathrm{F} \text { (L) } \pm 2^{\circ} \mathrm{F}(\mathrm{A}) \\
\pm 0.3^{\circ} \mathrm{F} \text { (L) } \pm 2.5^{\circ} \mathrm{F}(\mathrm{A}) \\
\pm \\
\pm \\
\pm\end{array}$ & $\begin{array}{l}5 \text { min } \\
5 \text { min } \\
5 \text { min } \\
5 \text { min } \\
\text { Daily st noon } \\
5 \text { min } \\
\text { Dsily st noon }\end{array}$ \\
\hline 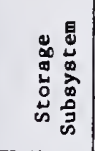 & $\begin{array}{r}T 200 \\
T 201 \\
\text { PD101 }\end{array}$ & $\begin{array}{l}\text { Storage Media Ave Temp } \\
\text { Storage Ambient Temp } \\
\text { Storage Press Diff (A1r) }\end{array}$ & $\begin{array}{l}60 \text { to } 230 \\
-20 \text { to } 120 \\
0 \text { to } 4 " \mathrm{H}_{2} \mathrm{O}\end{array}$ & $\begin{array}{l} \pm 2^{\circ} \mathrm{F} \\
\pm \\
\pm 2{ }^{\circ} \mathrm{F} \\
\pm 2 \% \mathrm{FS}\end{array}$ & $\begin{array}{l}\text { hr, } \\
\text { hr, } \\
h r,\end{array}$ \\
\hline 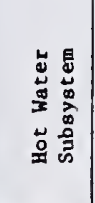 & $\begin{array}{r}\text { T301 } \\
\text { T300 } \\
\text { TD300 } \\
\text { TD301 } \\
\text { TD302 } \\
\text { W300 } \\
\text { W301 }\end{array}$ & $\begin{array}{l}\text { Makeup Water Temp } \\
\text { HX Inlet Temp } \\
\text { HX Temp Diff } \\
\text { Storage Temp Diff } \\
\text { Auxillary Temp Diff } \\
\text { Circulation Flow Rate } \\
\text { Load Flow Rate }\end{array}$ & $\begin{array}{l}30 \text { to } 90 \\
40 \text { to } 160 \\
0 \text { to } 50 \\
0 \text { to } 75 \\
0 \text { to } 100 \\
\text { varies } \\
\text { varies }\end{array}$ & $\begin{array}{l} \pm 0.5^{\circ} \mathrm{F} \\
\pm 0.5^{\circ} \mathrm{F} \\
\pm 0.3^{\circ} \mathrm{F} \\
\pm 0.3^{\circ} \mathrm{F} \\
\pm 0.3^{\circ} \mathrm{F} \\
\pm 2 \% \mathrm{FS} \\
\pm \\
\pm\end{array}$ & $\begin{array}{l}\text { Avg } / \mathrm{hr} \text {. } \\
5 \mathrm{~min} \\
5 \mathrm{~min} \\
5 \mathrm{~min} \\
5 \mathrm{~min} \text { or less } \\
5 \mathrm{~min} \\
5 \mathrm{~min}\end{array}$ \\
\hline 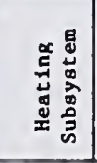 & $\begin{array}{r}T 400 \\
T D 400 \\
T D 401 \\
W 400 \\
T D 402\end{array}$ & $\begin{array}{l}\text { Load Return Temp } \\
\text { Storage Temp Diff } \\
\text { Auxillary Temp Diff } \\
\text { C1rculation Flow Rate } \\
\text { HTG Load Temp Diff }\end{array}$ & $\begin{array}{l}70 \text { to } 180 \\
0 \text { to } 50 \\
0 \text { to } 100 \\
\text { vartes } \\
0 \text { to } 100\end{array}$ & $\begin{array}{l} \pm 0.5^{\circ} \mathrm{F} \text { (L) } \pm 2^{\circ} \mathrm{F} \text { (A) } \\
\pm 0.3^{\circ} \mathrm{F} \text { (L) } \pm 2^{\circ} \mathrm{F} \text { (A) } \\
\pm 0.3^{\circ} \mathrm{F} \text { (L) } \pm 2^{\circ} \mathrm{F} \text { (A) } \\
\pm 2 \% \text { (L) } \pm 3 \overline{\%} \text { (A) FS } \\
\pm 0.3^{\circ} \mathrm{F} \text { (L) }\end{array}$ & $\begin{array}{l}5 \text { min } \\
5 \text { min } \\
5 \text { min } \\
5 \text { min } \\
5 \text { min }\end{array}$ \\
\hline 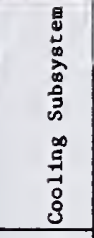 & $\begin{array}{r}\text { T500 } \\
\text { TD500 } \\
\text { T501 } \\
\text { TD501 } \\
\text { W500 } \\
\text { W501 }\end{array}$ & $\begin{array}{l}\text { Load HX Inlet Temp } \\
\text { Load HX Temp Diff } \\
\text { Cooling Tower Inlet Temp } \\
\text { Cooling Tower Temp Diff } \\
\text { Load HX Flow Rate } \\
\text { Cooling Tower Flow Rate }\end{array}$ & $\begin{array}{l}40 \text { to } 60 \\
0 \text { to } 75 \\
40 \text { to } 120 \\
0 \text { to } 20 \\
\text { varies } \\
\text { varies }\end{array}$ & $\begin{array}{l} \pm 0.5^{\circ} \mathrm{F} \\
\pm 0.3^{\circ} \mathrm{F} \\
\pm 0.5^{\circ} \mathrm{F} \\
\pm 0.3^{\circ} \mathrm{F} \\
\pm 2 \% \mathrm{FS} \\
\pm 2 \% \mathrm{FS}\end{array}$ & $\begin{array}{l}5 \mathrm{~min} \\
5 \mathrm{~min} \text { or less } \\
5 \mathrm{~min} \\
5 \mathrm{~min} \text { or less } \\
5 \mathrm{~min} \text { or } 1 \mathrm{ess}\end{array}$ \\
\hline 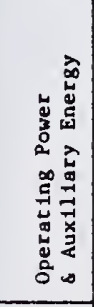 & $\begin{array}{l}\text { EP101 } \\
\text { EP } 301 \\
\text { EP401 } \\
\text { EP402 } \\
\text { EP403 } \\
\text { EP501 } \\
\text { EP300 } \\
\text { EP400 } \\
\text { F300 } \\
\text { F400 } \\
\text { F500 }\end{array}$ & $\begin{array}{l}\text { Collector Circ Pump Power } \\
\text { HW C1rc Pump Power } \\
\text { HTG Loop Clrc Pump Power } \\
\text { Bldg Fan Power } \\
\text { Heat Pump Compress Power } \\
\text { Absorp Chlller Oper Power } \\
\text { HW Elec Aux Power } \\
\text { HTG Elec Aux Power } \\
\text { HW Aux Fuel Flow } \\
\text { HTG Aux Fuel Flow } \\
\text { Cooling Aux Fuel Flow }\end{array}$ & $\begin{array}{l}\triangle K W \text { varies } \\
\Delta K W \text { varies } \\
\Delta K W \text { varies } \\
\Delta K W \text { vsries } \\
\Delta K W \text { varies } \\
\Delta K W \text { varies } \\
\Delta K W \text { varies } \\
\Delta K W \text { varies } \\
\text { varies } \\
\text { varles } \\
\text { varies }\end{array}$ & 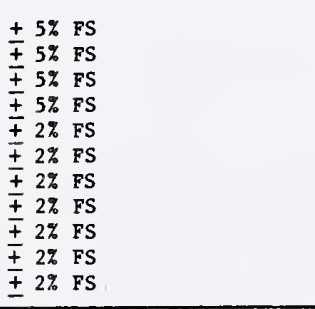 & $\begin{array}{l}5 \mathrm{~min} \\
5 \mathrm{~min} \\
5 \mathrm{~min} \\
5 \mathrm{~min} \\
5 \mathrm{~min} \text { or less } \\
5 \mathrm{~min} \text { or less } \\
5 \mathrm{~min} \text { or less } \\
5 \mathrm{~min} \text { or less } \\
5 \mathrm{~min} \text { or less } \\
5 \mathrm{~min} \text { or less } \\
5 \mathrm{~min} \text { or less }\end{array}$ \\
\hline 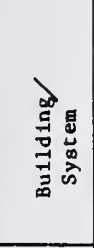 & $\begin{array}{r}T 600 \\
T D 600 \\
T 601 \\
T 602 \\
W 600 \\
W 601 \\
11 F 600 \\
T D 601\end{array}$ & $\begin{array}{l}\text { Bldg Return Air DB Temp } \\
\text { Supply/return A1r Temp Diff } \\
\text { Bldg Return air WB Temp } \\
\text { Bldg Supply Air WB Temp } \\
\text { Bullding A1r Flow Rate } \\
\text { Condensate Flow } \\
\text { Heat Flow Meter } \\
\text { HT Pump Cond \& Load HX Temp } \\
\text { Diff }\end{array}$ & $\begin{array}{l}50 \text { to } 90 \\
0 \text { to } 80 \\
20 \text { to } 80 \\
20 \text { to } 80 \\
\text { varies } \\
\text { varies } \\
0.25 \text { to } 30^{\circ} \mathrm{F} \Delta \mathrm{T} \\
0 \text { to } 80\end{array}$ & $\begin{array}{l} \pm 2^{\circ} \mathrm{F} \\
\pm 2^{\circ} \mathrm{F} \\
\pm \\
\pm 2^{\circ} \mathrm{F} \\
\pm 2^{\circ} \mathrm{F} \\
\pm 2 \% \mathrm{FS} \\
\pm 5 \% \\
\pm \\
\pm \\
\pm\end{array}$ & $\begin{array}{l}5 \mathrm{~min} \\
5 \mathrm{~min} \\
5 \mathrm{~min} \\
5 \mathrm{~min} \\
5 \mathrm{~min} \\
5 \mathrm{~min} \\
5 \mathrm{~min} \\
5 \mathrm{~min}\end{array}$ \\
\hline
\end{tabular}

Table 5 INSTRUMENTATION RANGE, ACCURACY AND MEASUREMENT FREQUENCY 

The performance factor data generated during the Demonstration Program will provide the basis to directly determine parameters such as energy saved, thermal efficiency and energy contributions to the various functional loads. However, to fulfill the program national goals the data will also be applied to determine correlations between building types, climates and heating and cooling system designs, to verify design methods and to establish reasonable performance goals.

Although exact system or subsystem comparisons must await the selection of specific solar system/building/climate combinations, examples of the format and general use of performance factors are presented as illustrations of how the data can be utilized.

8.1 Solar Energy System Thermal Performance

The technical selection of a solar system is based primarily on the thermal performance in terms of energy collected on monthly or annual/seasonal periods. Data such as shown in Figure 11 presents the amount of energy utilized for auxiliary energy as well as for the heating and cooling functions and the total energy supplied for a single site. Individual comparisons of solar energy systems (in similar climates) and collector areas can be made using this type of chart. For systems with varying collector size, the energy quantity can be expressed per unit collector area. Comparisons are made for each unique type of solar system to include air, liquid, active and passive. The data should be tabulated for monthly, seasonal and annual time periods to cover the following functional areas as appropriate.

Functions :

A. Hot Water

B. Space Heating

C. Space Cooling

D. Space Heating and Space Cooling

E. Space Heating and Hot Water

F. Space Heating and Cooling and Hot Water

G. Space Cooling and Hot Water

8.2 Solar Energy System Thermal Effectiveness

The effectiveness of a solar energy system to meet the total functional loads on a cumulative basis for each building type can be illustrated for a single site as shown in Figure 12. Comparison of the effectiveness of similar solar system types 



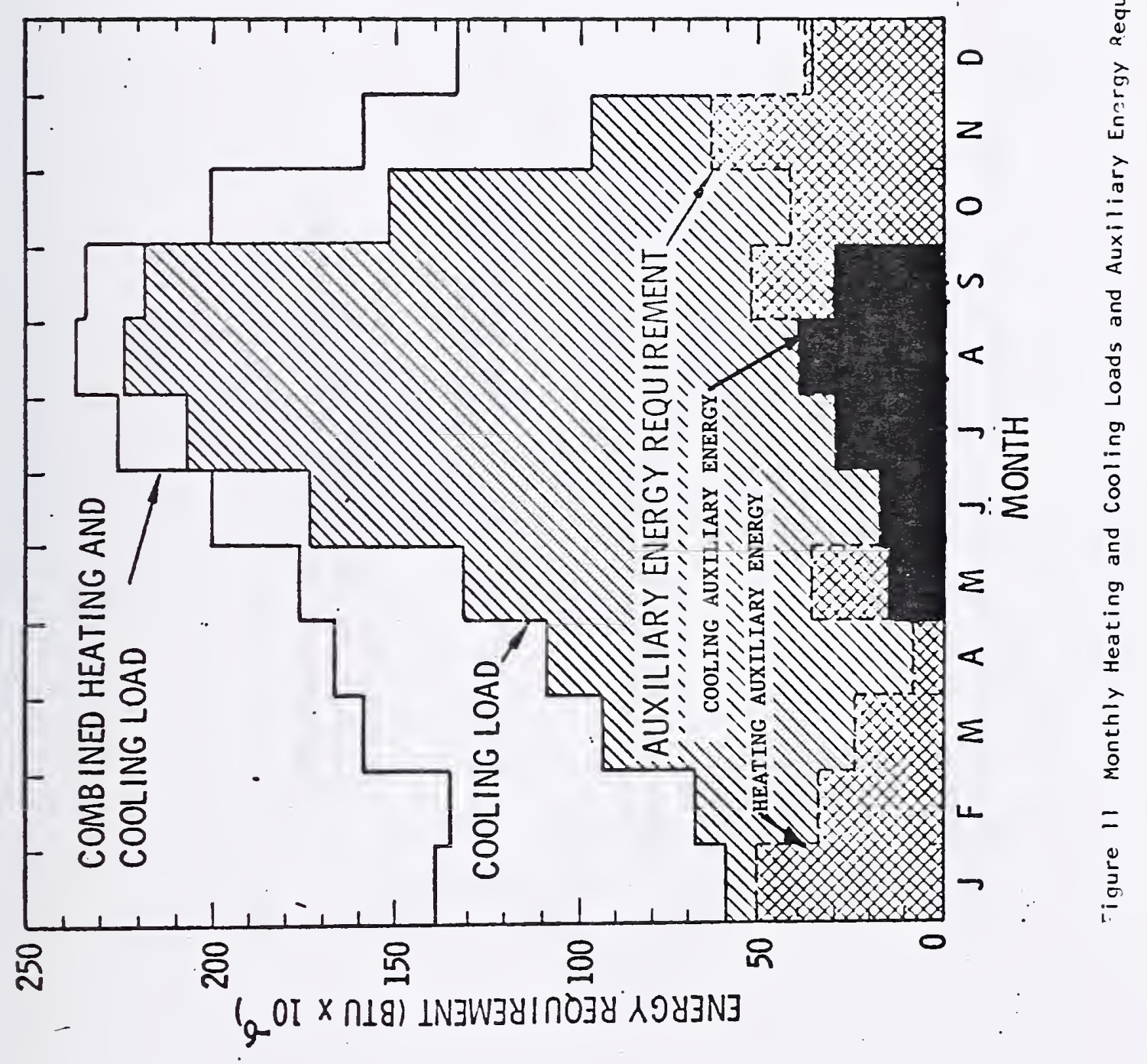





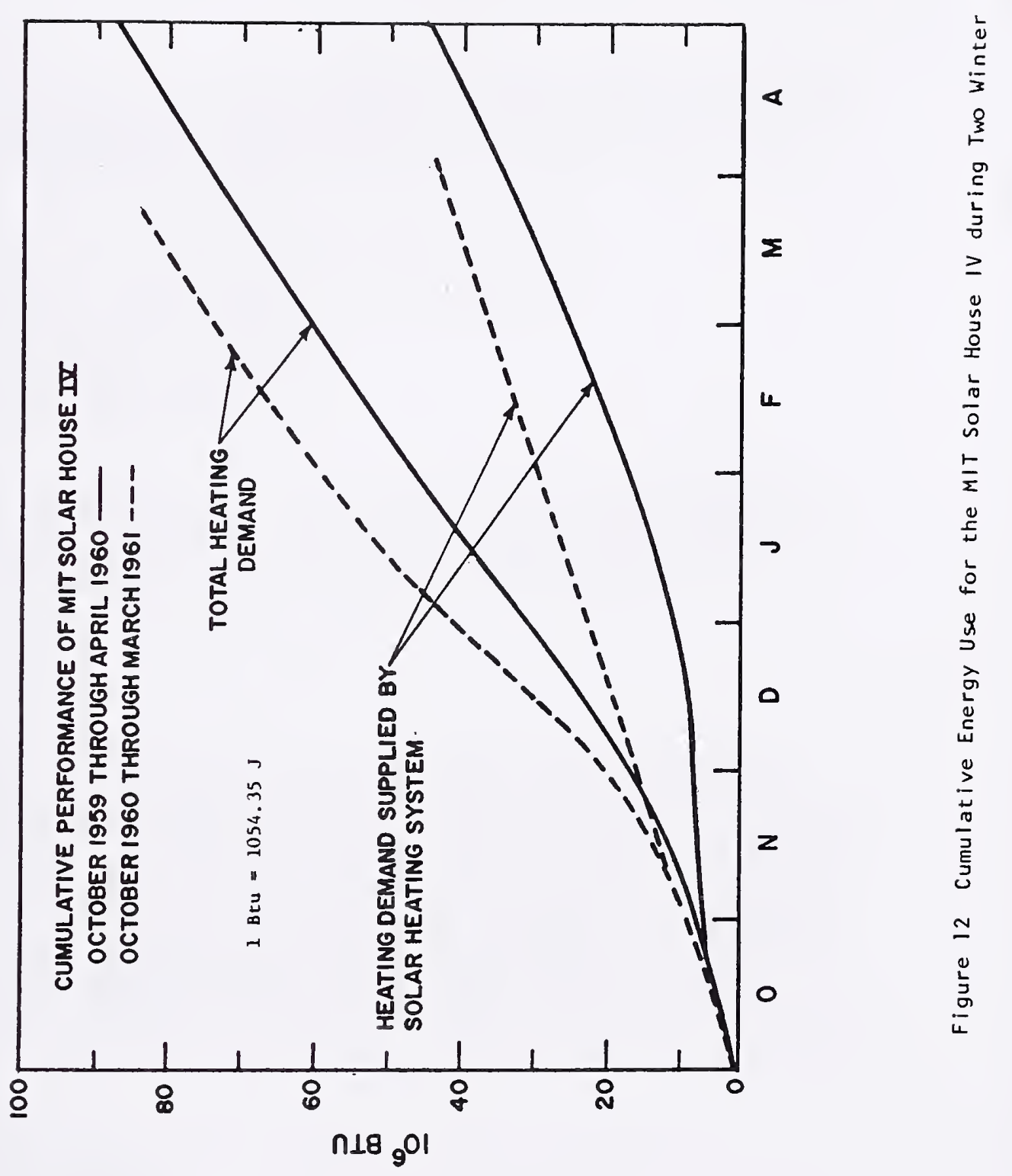



and climates on a seasonal basts is done by normalizing the building size and load per degree day of heating or cooling and presenting the data in tabular form for monthly, seasonal and annual time periods.

8.3 Analytical Predictions

The design of solar systems is currently based on analytical models and calculated performances. The demonstration program will provide the measured data for comparison with prediction of the following performance factors:

(1) Energy Delivered to Load (HW, heating, cooling, total)

(2) Solar Fraction of Load (HW, heating, cooling, total)

(3) Solar Energy Conversion Efficiency

(4) Energy Saved (Electric and Fossil)

(5) Collector Array Efficiency

Comparisons can be made on an individual site basis as illustrated in Figures 11 and 12 or tabular data for many sites can be used to determine standard deviations as a function of climate and building characteristics for monthly and annual periods.

8.4 Solar Collector Array

The greatest technical innovations are needed in the development of collectors, therefore, comprehensive characterization, measurement, and comparison of operating performance data for the many collector varieties will be necessary. Because of the influence of site integration and operating mode, comparison of preinstallation performance data for individual panels and the array performance, as illustrated in Figure 13, is desirable using instantaneous and dally, monthly and annual efficiencles. Other comparisons based upon design or material properties are listed as follows:

A. Flat Plate

1. Absorber Coating

a. Flat Black

b. Selective

2. Covers
a. Number
b. Material Type 



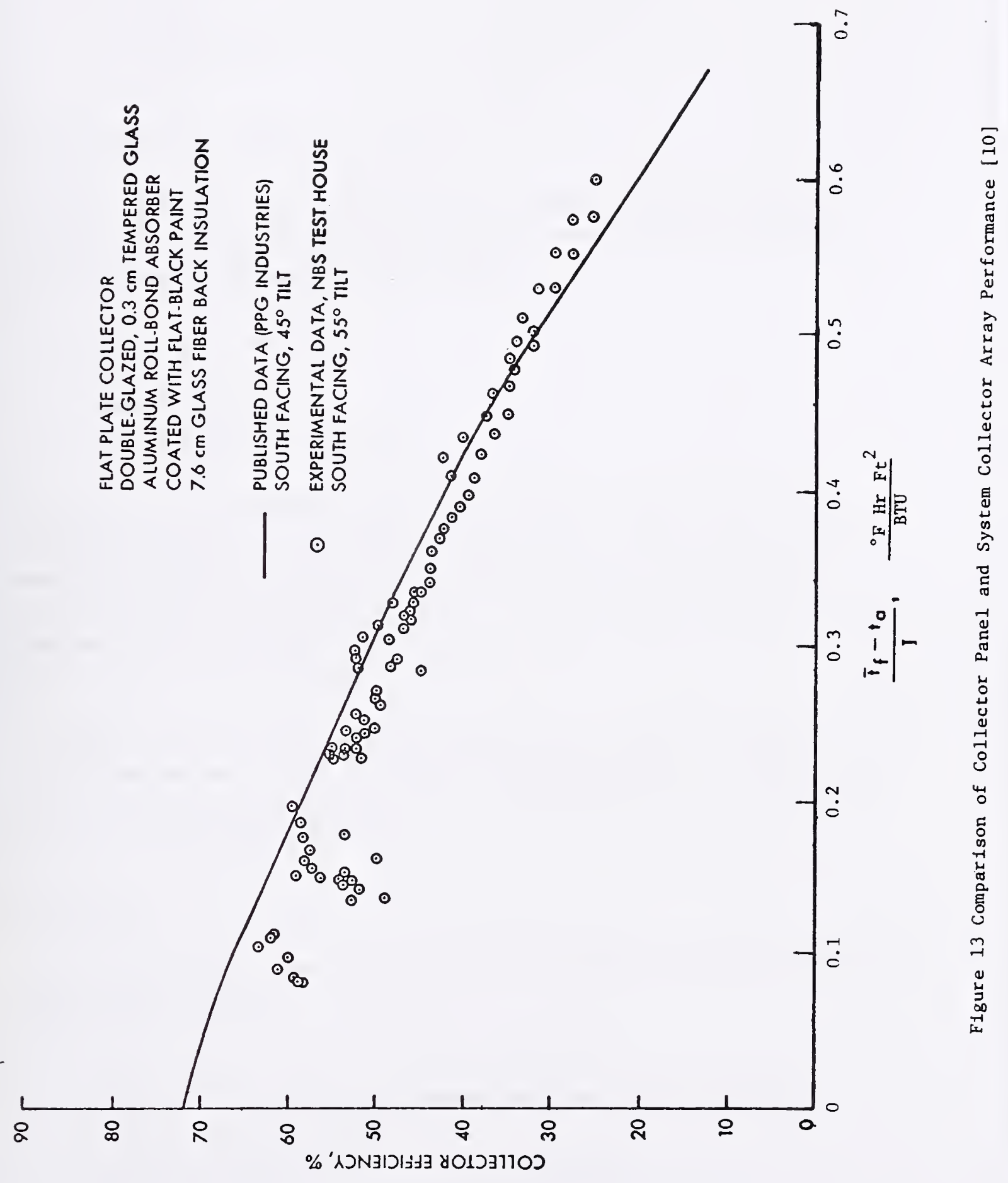



B. Concentrator

1. Cylindrical

2. Tubular

3. Trapezoidal

4. Other

\subsection{Component Predicted Performance}

It is not the intent of the test and evaluation program to develop components but the influence of the operating mode and environment will be determined on major subsystems or components as listed below:
A. Collector Array
B. Storage
C. Energy Transport
D. Energy Conversion Equipmert

An example of the comparisons in collector performance between predicted and experiment are shown in Table 6 for the three key heat transfer areas: (a) solar absorptance-transmittance product $(\alpha \tau)$; (b) heat removal efficiency factor $\left(F_{R}\right)$; and $(c)$ heat loss coefficient $\left(U_{L}\right)$. These values are obtained by reducing the collector performance data during selected periods of quasi-steady state to plot in the slope-intercept form.

An example of the use of the slope-intercept plot to illustrate collector material degradation is shown in Figure 14. Tabular values of a $\tau$ and $U_{L}$ for similar materials as a function of climatic exposure will reveal the differences related to environmental conditions.

\subsection{Climatic Data}

Climatic data measured during the program will be reported for hourly, monthly and annual data as follows:
A. Total solar radiation at collector tilt angle
B. Direct solar radiation at collector tilt angle for selected sites
C. Ambient dry bulb temperature
D. Ambient wet bulb temperature at selected sites
E. Wind direction and velocity at selected sites

These data will be tabulated and plots indicating variations over the continental United States will be available. 



\begin{tabular}{|c|c|c|c|c|c|}
\hline & 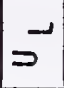 & $\underset{\mathscr{\theta}}{\mathscr{\theta}}$ & $\underset{0}{\tilde{0}}$ & চִ & $\stackrel{m}{\sigma}$ \\
\hline 흥 & ${ }^{\infty}$ & $\begin{array}{l}8 \\
0 \\
0\end{array}$ & $\begin{array}{l}8 \\
8 \\
0\end{array}$ & $\begin{array}{l}\stackrel{5}{9} \\
0\end{array}$ & 它 \\
\hline 㟧 & $5^{\circ}$ & $\begin{array}{l}F \\
0\end{array}$ & $\stackrel{E}{E}$ & $\begin{array}{l}\mathscr{\mathscr { O }} \\
0 \\
0\end{array}$ & $\begin{array}{l}\tilde{\infty} \\
\infty \\
0 \\
0\end{array}$ \\
\hline 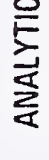 & 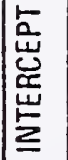 & $\frac{\pi}{5}$ & $\underset{0}{\Delta}$ & $\frac{T}{0}$ & $\begin{array}{l}\stackrel{0}{0} \\
0 \\
0\end{array}$ \\
\hline & 5 & $\stackrel{\mathscr{0}}{0}$ & $\begin{array}{l}\infty \\
\stackrel{\infty}{0} \\
0\end{array}$ & $\underset{0}{\infty}$ & $\stackrel{2}{-}$ \\
\hline 岕 & $u^{\infty}$ & $\begin{array}{l}0 \\
0 \\
0 \\
0\end{array}$ & $\begin{array}{l}8 \\
0 \\
0\end{array}$ & D্ & 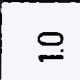 \\
\hline$\vec{s}$ & $\begin{array}{l}N^{\infty} \\
0\end{array}$ & 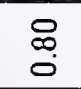 & $\begin{array}{c}\tilde{N} \\
0 \\
0\end{array}$ & 車 & $\begin{array}{l}\mathscr{g} \\
0 \\
0\end{array}$ \\
\hline 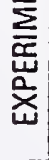 & 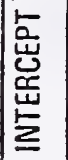 & $\underset{\sigma}{\pi}$ & $\begin{array}{l}\infty \\
0 \\
0\end{array}$ & $\underset{\mathscr{\sigma}}{\tilde{O}}$ & 总 \\
\hline 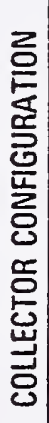 & & 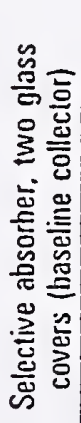 & 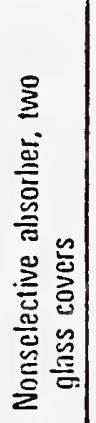 & 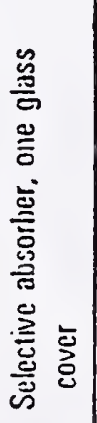 & 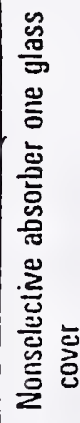 \\
\hline
\end{tabular}





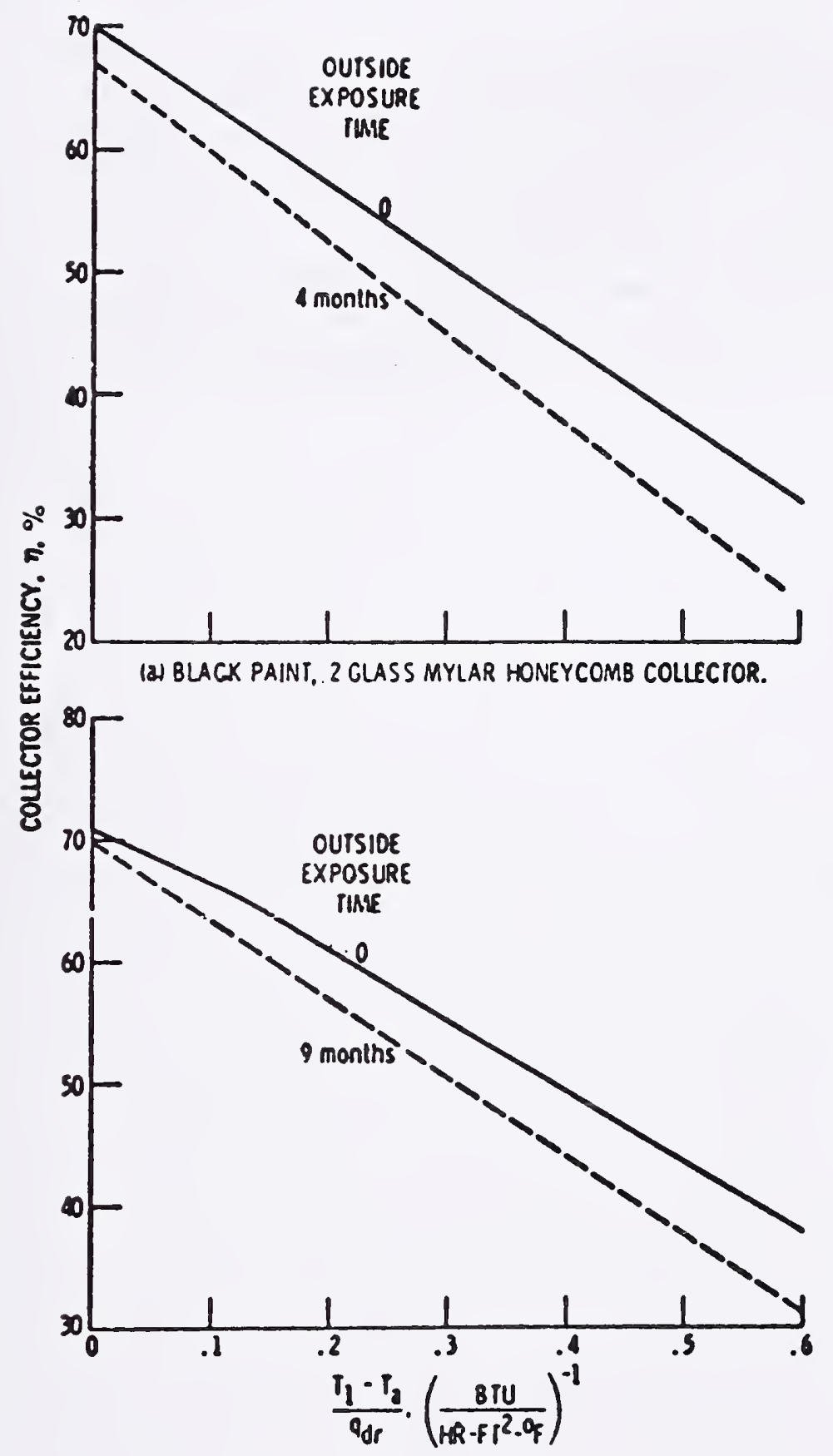

(O) BLACK-NICKEL, 2 CLASS COLLECTOR (5).

Figure 14 Illustrations of Detecting Collector Degradation From Thermal Performance Measurements. 

8.

Table 7 lists a number of data correlations and evaluations possible with the avallable measurements and calculated performance factors. Due to the large number of bullding types solar energy systems and climatic regions, it is obvious that computerized data reduction will be necessary to perform the necessary evaluation. It is therefore recommended that additional study of the data format and computerized data reduction requirements be under taken to more efficlently Implement the various correlations studies planned and to assure avallability of the results to the potential users on a timely basis. 



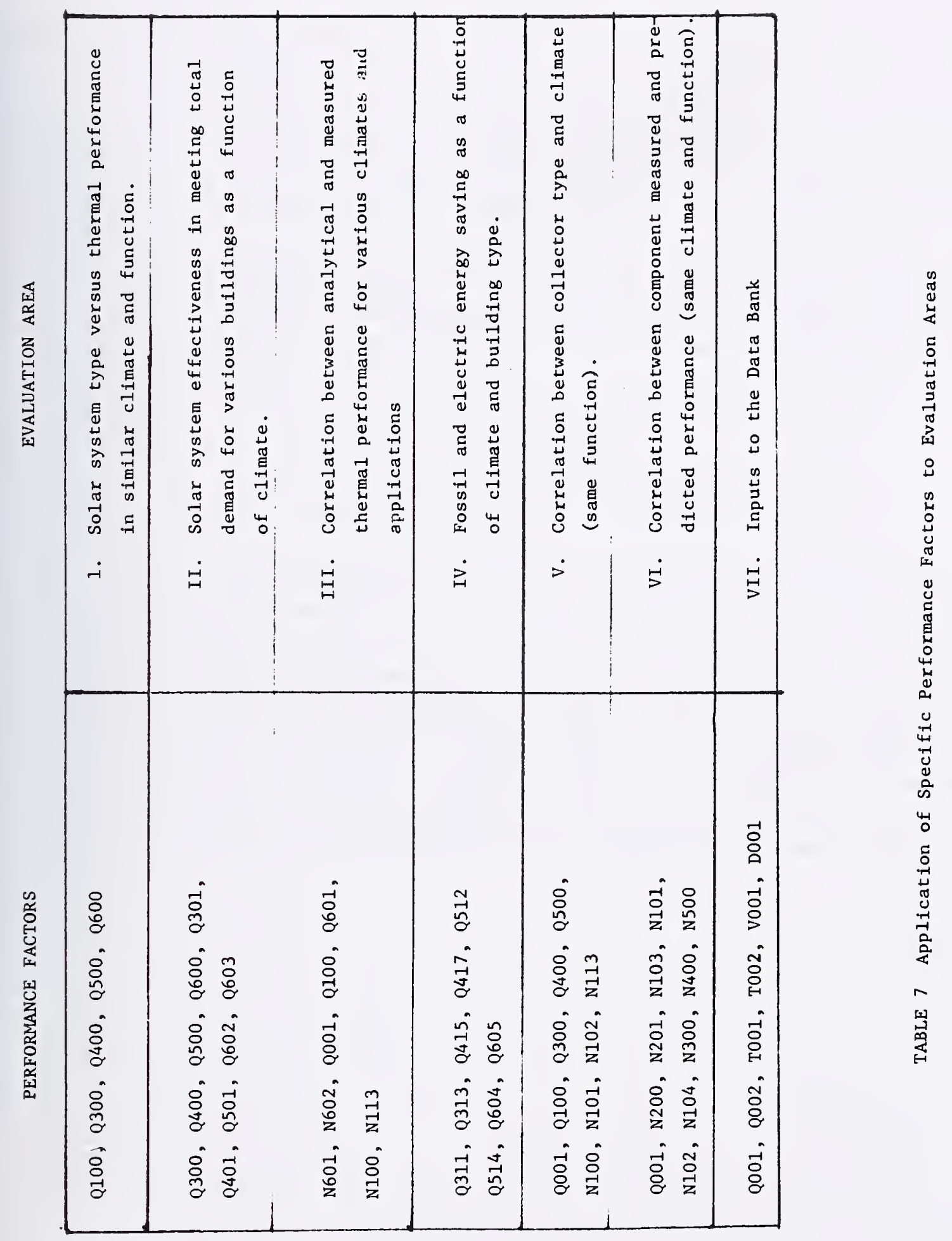



The quantity and type of measurements described reflect a level of instrumentation that will provide a capability for full evaluation of each solar energy system and for each building, partial evaluation of most components performance and measurement of local climatic data.

To reduce instrumentation costs, an assessment of the significance of the performance factors should be made for each proposed demonstration unit. The four factors considered essential for the performance of solar energy systems are:

- conventional energy saved by the solar energy system

- solar fraction of total load

- solar energy system conversion efficiency

- solar energy collected per unit collector area.

The primary tool used to determine the data requirements and the selection of instrumentation is the analytical heat balances. Sufficient heat balance calculations are required to equate the total energy input to the total energy output for the subsystem or component plus an acceptable heat loss to provide an energy balance closure of about ten percent. Errors greater than approximately ten percent for active systems and fifteen percent for passive systems will not permit useful comparison between different systems. Error analysis of most performance evaluation factors for active solar energy systems have shown that the experimental data can be obtained with accuracy of about \pm 5 percent using commercially available sensors. 

1. National Plan for Solar Heating and Cooling (Residential and Commercial Applications) ERDA-23A, October 1975 .

2. ASHRAE Standard 90-75, "Energy Conservation in New Building Design," 1975. Available American Soclety of Heating and Refrigerating and Air Conditioning Engineers, Inc., 345 East 47 th Street, New York, N.Y. 10017

3. "Procedures for Determining Heating and Cooling Loads for Computerized Energy Calculations", The Task Group on Energy Requirements for Heating and Cooling, ASHRAE, 345 East 47 th Street, New York, 1971.

4. T. Kusuda, "NBSLD, Computer Program for Heating and Cooling Loads in Buildings", NBS Report NBSIR 74-574, November 1974.

5. Personal Communication, Dr. Dan S. Ward, Colorado State University, Ft. Collins, Colorado; and Study on "Instrumentation Type, Range, Accuracy, and Measurement Frequency Required to Monitor and Evaluate the Thermal Effectiveness of Solar Heating and Cooling and Hot Water Systems", NBS Purchase Order No. 608692, March 1976.

6. ASHRAE Standard 93-P "Method of Testing for Rating Solar Collectors Based on Thermal Performance", August 1976 (Avallable ibid ASHRAE).

7. G. E. Kelly, "Potential of Air-to-Air Heat Pumps for Energy Conservation in Residential Bulldings", NATO Advanced Study Institutes, Series E: Applied Science No. 15, Noordoff-leyden, 1976 (Avallable from National Bureau of Standards, Washington, D C 20234)

8. Persona1 Communication, Dr. William H. Klein, Radiation Biology Laboratory, Smithsonian Institution, Rockville, Maryland, January 1976.

9. Engebretson, C. D. , The Use of Solar Energy for Space Heating, M. I. T. Solar House IV, "Proceedings of the United Nations Conference on New Sources of Energy", Rome, August 21-31, 1961, Vol. 5 III e.2 pp. 159-172.

10. J. E. Hill, T. E. Richtmyer and J. Jenkins, "Initial Test Results for a Solar Cooled Townhouse in the Mid-Atlantic Region", National Bureau of Standards, Washington, D C 20234, Presented at ASHRAE Semi-Annual Meeting, June 1976.

11. J. W. Ramsey, J. T. Borzoni and T. H. Holland, "Development of Flat-P1ate Solar Collectors for the Heating and Cooling of Bulldings, "NASA CR-134804, June 1975. 

NBS-114A (REV. 7-73)

\begin{tabular}{|c|c|c|}
\hline $\begin{array}{l}\text { U.S. OEPT. OF COMM. } \\
\text { BIBLIOGRAPHIC DATA } \\
\text { SHEET }\end{array}$ & $\begin{array}{l}\text { 1. PUIBI.IC.ATION OR R I:PORT NO. } \\
\text { NBSIR 76-1137 }\end{array}$ & 3. Recipient's Accession No. \\
\hline \multirow{2}{*}{\multicolumn{2}{|c|}{$\begin{array}{l}\text { 4. TITI.E AND SUBTITI.F } \\
\text { Thermal Data Requirements and Performance Evaluation } \\
\text { Procedures for the National Solar Heating and Cooling } \\
\text { Demonstration Program }\end{array}$}} & 5. Publication Date \\
\hline & & 6. Performing Organization Code \\
\hline $\begin{array}{l}\text { 7. AUTHOR(S) } \\
\text { E. Streed, M. McCa }\end{array}$ & D. Waksman, J. Hebrank, T. Richtmyer & 8. Performing Organ. Report No. \\
\hline \multirow{2}{*}{\multicolumn{2}{|c|}{$\begin{array}{l}\text { 9. PERFORMING ORGANIZATION NAMF. AND ADDRESS } \\
\text { NATIONAL BUREAU OF ST ANDARDS } \\
\text { DEPARTMENT OF COMMERCE } \\
\text { WASHINGTON, D.C. } 20234\end{array}$}} & $\begin{array}{l}\text { 10. Project/Task/Work Unit No. } \\
4606500 \text { and } 4606505\end{array}$ \\
\hline & & $\begin{array}{l}\text { 11. Contract/Grant No. } \\
\text { ERDA }-49(1)-3800\end{array}$ \\
\hline \multicolumn{2}{|c|}{$\begin{array}{l}\text { 12. Sponsoring Organization Name and Complete Address (Street, City, State, ZIP) } \\
\text { Energy Research \& Development Admin. Dept. of Housing and } \\
\text { Division of Solar Energy }\end{array}$} & $\begin{array}{l}\text { 13. Type of Report \& Period } \\
\text { Covered } \\
\text { Interim }\end{array}$ \\
\hline Washington, DC 2 & 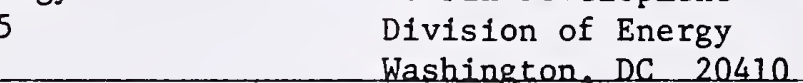 & 14. Sponsoring Agency Code \\
\hline
\end{tabular}

16. ABSTRACT (A 200 word or less factual summary of most significant information. If document includes a significant bibliography or literature survey, mention it here.)

This report presents the results of a study to determine the data requirements and performance evaluation factors to be used in the National Solar Heating and Cooling Demonstration Program. Solar energy systems used for heating hot water, space heating, and space cooling have been considered and specific measurements and analytical procedures have been recommended to determine the thermal effectiveness for daily, monthly, seasonal, or annual operating periods. The sensor accuracy and sampling rate effects on measurement uncertainty for several performance factors is presented. Application of the individual performance factors for the comparison of subsystem and system thermal performance as a function of building type and climatic region is discussed.

17. KEY WORDS (six to twelve entries; alphabetical order; capitalize only the first letter of the first key word unless a proper name; separated. $v$ semicolons)

Data requirements; measurement uncertainty; solar energy systems evaluation; thermal performance.

18. AVAILABILITY Unlimited

$\neg$ For Official Distribution. Do Not Release to NTIS

Order From Sup. of Doc., U.S. Government Printing Office Washington, D.C. 20402, SD Cat. No. C13

$x$ Order From National Technical Information Service (NTIS) Springfield, Virginia 22151

\begin{tabular}{|l|c|}
\hline $\begin{array}{l}\text { 19. SECURITY CLASS } \\
\text { (THIS REPURT) }\end{array}$ & 21. NO. OF PAGES \\
UNCL ASSIFIED & 85 \\
\hline $\begin{array}{l}\text { 20. SECURITY CLASS } \\
\text { (THIS PAGE) }\end{array}$ & 22. Price \\
UNCLASSIFIED & $\$ 5.00$ \\
\hline
\end{tabular}



